\title{
A nonlinear Bismut-Elworthy formula for HJB equations with quadratic Hamiltonian in Banach spaces
}

\author{
Davide ADDONA* ${ }^{1}$, Elena BANDINI ${ }^{\dagger 1}$ and Federica MASIERO $\$ 1$ \\ ${ }^{1}$ Dipartimento di Matematica e Applicazioni, Università di Milano-Bicocca, Milano, Italy
}

\begin{abstract}
We consider a Backward Stochastic Differential Equation (BSDE for short) in a Markovian framework for the pair of processes $(Y, Z)$, with generator with quadratic growth with respect to $Z$. The forward equation is an evolution equation in an abstract Banach space. We prove an analogue of the Bismut-Elworty formula when the diffusion operator has a pseudo-inverse not necessarily bounded and when the generator has quadratic growth with respect to $Z$. In particular, our model covers the case of the heat equation in space dimension greater than or equal to 2 . We apply these results to solve semilinear Kolmogorov equations for the unknown $v$, with nonlinear term with quadratic growth with respect to $\nabla v$ and final condition only bounded and continuous, and to solve stochastic optimal control problems with quadratic growth.
\end{abstract}

Keywords: Stochastic heat equation in 2 and 3 dimensions, nonlinear Bismut-Elworthy formula, quadratic Backward Stochastic Differential Equation, Hamilton Jacobi Bellman equation.

MSC 2010: 60H10; 60H30; 93E20; 35Q93.

\section{Introduction}

In this paper we deal with Markovian BSDEs whose generator has quadratic growth with respect to $Z$, and we generalize to this framework the Bismut-Elworthy type formula introduced in [10], where the Lispchitz case was studied. More precisely, our BSDE is related to a forward stochastic differential equation of the form

$$
\left\{\begin{array}{l}
d X_{\tau}^{t, x}=A X_{\tau}^{t, x} d \tau+F\left(X_{\tau}^{t, x}\right) d \tau+(-A)^{-\alpha} d W_{\tau}, \quad \tau \in[t, T], \\
X_{t}^{t, x}=x \in E
\end{array}\right.
$$

where $E$ is a Banach space which is continuously and densely embedded in a real and separable Hilbert space $H$. The operator $A$ is the generator of a contraction analytic semigroup in $H$, which turns out to be strongly continuous or analytic in $E$, and $\left\{W_{\tau}, \tau \geq 0\right\}$ is a cylindrical Wiener process in $H$. We assume that the stochastic convolution

$$
w_{A}(\tau)=\int_{0}^{\tau} e^{(\tau-s) A}(-A)^{-\alpha} d W_{s}
$$

is well defined as a Gaussian process in $H$, and that it admits an $E$-continuous version.

\footnotetext{
*davide.addona@unimib.it

†elena.bandini@unimib.it

${ }^{\ddagger}$ federica.masiero@unimib.it
} 
The presence of the diffusion operator $(-A)^{-\alpha}$ in (1.1) allows us to deal with stochastic heat equations in 2 and 3 space dimensions, while stochastic heat equations in one space dimension can be considered without any regularization of the white noise, that is in the case with $\alpha=0$. Moreover, we consider dissipative maps $F$ in (1.1) in order to have more generality in the structure of the equation. Notice that, under this latter assumption, $F$ is well defined only on the Banach space $E$, while it is not even defined on the whole Hilbert space $H$; this is a natural situation arising in many evolution equations, see e.g. [6] and $[4]$.

The solution of equation (1.1) will be denoted by $X$, or also by $X^{t, x}$, to stress the dependence on the initial conditions, and the transition semigroup related to $X^{t, x}$ will be denoted by

$$
P_{t, \tau}[\phi](x):=\mathbb{E} \phi\left(X_{\tau}^{t, x}\right), \quad \phi \in B_{b}(E)
$$

At least formally, the generator of $P_{t, \tau}$ is the second order differential operator

$$
(\mathscr{L} f)(x)=\frac{1}{2}\left(\operatorname{Tr}\left((-A)^{-\alpha}\left(-A^{*}\right)^{-\alpha} \nabla^{2} f\right)(x)+\langle A x, \nabla f(x)\rangle+\langle F(x), \nabla f(x)\rangle .\right.
$$

This is the link with the solution, in mild sense, of the semilinear Kolmogorov equation in $E$ (see e.g. $[5])$ :

$$
\left\{\begin{array}{l}
\frac{\partial v}{\partial t}(t, x)=-\mathscr{L} v(t, x)+\psi\left(t, x, v(t, x), \nabla v(t, x)(-A)^{-\alpha}\right), \quad t \in[0, T], x \in E, \\
v(T, x)=\phi(x) .
\end{array}\right.
$$

We recall that by mild solution of equation (1.2) we mean a bounded and continuous function $v$ : $[0, T] \times H \rightarrow H$, once Gâteaux differentiable with respect to $x$, and satisfying the integral equality

$$
v(t, x)=P_{t, T}[\phi](x)+\int_{t}^{T} P_{t, s}\left[\psi\left(s, \cdot, v(s, \cdot), \nabla v(s, \cdot)(-A)^{-\alpha}\right)\right](x) d s, \quad t \in[0, T], x \in E .
$$

Second order differential equations are a widely studied topic in the literature, see e.g. [5]. In the case of $\psi$ only locally Lipschitz continuous, we cite [15], [22], [20] and also [21], where in particular the quadratic case is studied with datum $\phi$ only continuous. We also mention the monograph [3], where semilinear Kolmogorov equations related to forward equations of reaction diffusion type more general than the one considered here are studied, but requiring Lipschitz continuity of the final datum.

We will consider equation (1.2) under the assumptions that the final datum $\phi$ is bounded and continuous, and that $\psi$ has quadratic growth with respect to the derivative $\nabla v(-A)^{-\alpha}$. In order to prove existence and uniqueness of a mild solution of the form (1.3) for the Kolmogorov equation (1.2), we aim at representing this mild solution in terms of a Markovian BSDE of the form

$$
\left\{\begin{array}{l}
d Y_{\tau}=-\psi\left(\tau, X_{\tau}, Y_{\tau}, Z_{\tau}\right) d \tau+Z_{\tau} d W_{\tau} \\
Y_{T}=\phi\left(X_{T}\right)
\end{array}\right.
$$

We recall that, in order to solve partial differential equations by means of BSDEs, one of the crucial tasks is the identification of $Z$ with the derivative of $Y$ taken in the directions of the diffusion operator. In this regard, we refer to the seminal paper [23] for the finite dimensional case, and to [11] for the infinite dimensional extension in Hilbert spaces: in both papers the driver $\psi$ is Lipschitz continuous in $Y$ and in $Z$, and $\psi$ and $\phi$ are differentiable. We also mention [19], where an extension to the Banach space case is studied with the same assumptions of Lipschitz continuity and differentiability on the data.

In the present paper we do not make differentiability assumptions on the coefficients: thank to a variant of the nonlinear Bismut-Elworthy formula for BSDEs introduced in [10], we are still able to prove that the solution of the BSDE (1.4) gives the mild solution of the Kolmogorov equation (1.2). Bismut-Elworthy formulas for the transition semigroup of equations of type (1.1) with invertible diffusion operator are a classical topic in the literature, see e.g. [5]. In [3] the case of an operator like the one 
in (1.1), with pseudo-inverse which is not necessarily bounded, is also considered. According to these classical Bismut formulas, for every $0 \leq t<\tau \leq T, x \in H, h \in H$, and for every bounded and continuous real function $f$ defined on $H$, one has

$$
\left\langle\nabla_{x} P_{t, \tau}[f](x), h\right\rangle=\mathbb{E} f\left(X_{\tau}^{t, x}\right) U_{\tau}^{h, t, x},
$$

where $\left(G\left(r, X_{r}^{t, x}\right)\right.$ being the general diffusion operator)

$$
U_{\tau}^{h, t, x}:=\frac{1}{\tau-t} \int_{t}^{\tau}\left\langle G^{-1}\left(r, X_{r}^{t, x}\right) \nabla_{x} X_{r}^{t, x} h, d W_{r}\right\rangle .
$$

In [10] a nonlinear Bismut-Elworthy formula for the process $Y$ solution of the BSDE (1.4) is proved when $\psi$ is Lipschitz continuous with respect to $Z$ and the process $X$ takes its values in a Hilbert space $H$. According to this formula, for $0 \leq t<\tau \leq T, x \in H$, for every direction $h \in H$,

$$
\mathbb{E}\left[\nabla_{x} Y_{\tau}^{t, x} h\right]=\mathbb{E}\left[\int_{\tau}^{T} \psi\left(r, X_{r}^{t, x}, Y_{r}^{t, x}, Z_{r}^{t, x}\right) U_{r}^{h, t, x} d r\right]+\mathbb{E}\left[\phi\left(X_{T}^{t, x}\right) U_{T}^{h, t, x}\right] .
$$

Formula (1.6) is used in [10] to solve a semilinear Kolmogorov equation of the form of (1.2). When the Hamiltonian function $\psi$ is Lipschitz continuous with respect to the derivative of $v$, semilinear Kolmogorov equations of the type of (1.2) can be solved also by using the estimates coming from the classical Bismut formulas (1.5) and by a fixed point argument, see e.g. [3], [5], [14]. In the quadratic case this procedure does not work anymore: for this reason, nonlinear versions of Bismut-Elworthy formulas, that give an alternative way to solve equations like (1.2), are particularly interesting in such a framework. In [21], a nonlinear version of the Bismut-Elworthy formula has been provided and has been applied to semilinear Kolmogorov equations of the type of (1.2), with quadratic hamiltonian, and in a Hilbert space.

In the present paper, we generalize (1.6) to the Banach space framework, and to the case of diffusion operator $(-A)^{-\alpha}$ that has unbounded pseudo-inverse operator. In this context, the nonlinear Bismut formula (1.6) has its own independent interest, and moreover it allows to solve the Kolmogorov equation with Hamiltonian function quadratic with respect to $\nabla v(-A)^{-\alpha}$. We first provide an analogous of the nonlinear Bismut formula given in [10] in the case of Banach space framework and Lispchitz continuous generator. Then, we prove a nonlinear Bismut formula in the quadratic case when $\psi$ and $\phi$ are differentiable. To this end, denoted by $\left(Y^{t, x}, Z^{t, x}\right)$ a solution to the Markovian BSDE (1.4) and assuming that $\phi$ and $\psi$ are differentiable, the two main ingredients are the identification

$$
Z_{t}^{t, x}=\nabla_{x} Y_{t}^{t, x}(-A)^{-\alpha}, \quad t \in[0, T], x \in E,
$$

and an a priori estimate on $Z^{t, x}$ of the form ( $C$ being a constant depending on $\left.t, T, A, F,\|\phi\|_{\infty}\right)$

$$
\left|Z_{t}^{t, x}\right|_{H} \leq C(T-t)^{-1 / 2},
$$

which is obtained with techniques similar to the ones used in [21], see also [7] and [26]. Both (1.7) and (1.8) are new in the Banach space framework and in the case of quadratic generator with respect to $z$. Finally, differentiability assumptions are removed by an approximation procedure, obtained by suitably generalizing the one introduced in [25].

Our results can be applied to a stochastic optimal control problem consisting in minimizing a cost functional of the form

$$
J(t, x, u)=\left[\mathbb{E} \int_{t}^{T} l\left(s, X_{s}^{u}, u_{s}\right) d s+\mathbb{E} \phi\left(X_{T}^{u}\right)\right]
$$

over all the admissible controls $u$ taking values in $H$ and not necessarily bounded. Here $l$ has quadratic growth with respect to $u$, and $X^{u}$ is the solution of the controlled state equation

$$
\left\{\begin{array}{l}
d X_{\tau}^{u}=A X_{\tau}^{u} d \tau+F\left(X_{\tau}^{u}\right) d \tau+Q u_{\tau} d \tau+(-A)^{-\alpha} d W_{\tau}, \quad \tau \in[t, T] \\
X_{t}^{u}=x
\end{array}\right.
$$


with $Q=I$ or $Q=(-A)^{-\alpha}$. The aim of this latter part of the work is to characterize the value function as the solution of the associated Hamilton Jacobi Bellman (HJB in the following) equation, and to provide a feedback law for optimal controls. If $Q=(-A)^{-\alpha}$, namely when the controls affect the system only through the noise (the so called structure condition holds true), the optimal control problem (1.9) can be completely solved, see Theorem 6.10. When $Q=I$, the optimal control problem can be completely solved by restricting ourselves to the class of more regular controls taking values in $D\left((-A)^{-\alpha}\right)$, see Theorem 6.15. In the general case of $Q=I$ and $H$-valued controls, we are able to provide an " $\varepsilon$-optimal solution" of the problem in the sense that the value function can be approximated by a sequence of functions which are solutions of approximating HJB equations, and we can obtain an $\varepsilon$-optimal control in feedback form, see Theorem 6.25 .

The paper is organized as follows: in Section 2 we fix the notations and we give the results on the forward process. In Section 3 we introduce the forward backward system: here the main results are the identification (1.7) of $Z_{t}^{t, x}$ with $\nabla Y_{t}^{t, x}(-A)^{-\alpha}$, which is new in the case of $\psi$ quadratic with respect to $z$ and in the Banach space framework, and the a priori estimate (1.8) on $Z$ not involving derivatives of the coefficients of the BSDE. In Section 4 we give the nonlinear Bismut formula (1.6) in the Banach space $E$ and with $\psi$ Lipschitz continuous with respect to $z$, then in Section 5 we extend formula (1.6) to the case of $\psi$ quadratic with respect to $z$. In both Sections 4 and 5, the Bismut formula is applied to solve the corresponding semilinear Kolmogorov equation (1.2). Finally in Section 6 we apply the previous results to solve the stochastic optimal control problem (1.9).

\section{Notations and preliminary results on the forward process}

We assume that $E$ is a real and separable Banach space which admits a Schauder basis, and that $E$ is continuously and densely embedded in a real and separable Hilbert space $H$. $E$ and $H$ are respectively endowed with the norms $|\cdot|_{E}$ and $|\cdot|_{H}$. We fix a complete probability space $(\Omega, \mathscr{F}, \mathbb{P})$ endowed with a filtration $\left\{\mathscr{F}_{t}, t \geq 0\right\}$ satisfying the usual conditions.

We list below some notations that are used in the paper. Let $K$ be a given Banach space endowed with the norm $|\cdot|_{K}$. For any $p, q \in[1, \infty)$ and any $t \in[0, T]$, we set

- $L^{p}(0, T ; K)$ the space of $K$-valued measurable functions defined on $[0, T]$, normed by

$$
\|f\|_{L^{p}(0, T ; K)}:=\left(\int_{0}^{T}\left|f_{s}\right|_{K}^{p} d s\right)^{1 / p} \text {. }
$$

- $L^{q}\left(\Omega ; L^{p}(0, T ; K)\right)$ the space of adapted processes $\left(u_{s}\right)_{s \in[0, T]}$, defined on $[0, T]$ and with values in $K$, normed by

$$
\|u\|_{L^{q}\left(\Omega ; L^{p}(0, T ; K)\right)}:=\left[\mathbb{E}\left(\int_{0}^{T}\left|u_{s}\right|_{K}^{p} d s\right)^{q / p}\right]^{1 / q} .
$$

- $\mathscr{S}^{p}((t, T] ; K)$ (resp. $\left.\mathscr{S}^{p}([t, T] ; K)\right)$ the space of all adapted processes $\left(X_{s}\right)_{s \in[t, T]}$, continuous on $(t, T]$ (resp. on $[t, T])$ and with values in $K$, normed by

$$
\|X\|_{\mathscr{S}^{p}([t, T] ; K)}=\|X\|_{\mathscr{S}^{p}((t, T] ; K)}:=\mathbb{E}\left[\sup _{s \in[t, T]}\left|X_{s}\right|_{K}^{p}\right]^{1 / p}
$$

If $K=\mathbb{R}$ we simply write $\mathscr{S}^{p}([t, T])$.

- $\mathscr{M}^{p}([t, T] ; K)$ the space of all predictable processes $\left(Z_{s}\right)_{s \in[t, T]}$ with values in $K$ normed by

$$
\|Z\|_{\mathscr{M}^{p}([t, T] ; K)}:=\mathbb{E}\left[\left(\int_{t}^{T}\left|Z_{s}\right|_{K}^{2} d s\right)^{p / 2}\right]^{1 / p} .
$$


If $K=\mathbb{R}$ we simply write $\mathscr{M}^{p}([t, T])$.

We denote by $L(E, K)$ the space of all bounded linear operators from $E$ to $K$, endowed with the usual operator norm. $E^{*}$ denotes the dual space of $E$, and $\langle\cdot, \cdot\rangle_{E \times E^{*}}$ denotes the duality between $E$ and $E^{*}$. We say that a function $f: E \rightarrow K$ belongs to the class $\mathscr{G}^{1}(E, K)$ if $f$ is continuous and Gâteaux differentiable on $E$ and if the gradient $\nabla f: E \rightarrow L(E, K)$ is strongly continuous. If $K=\mathbb{R}$ we simply write $\mathscr{G}^{1}(E)$. We say that $f:[0, T] \times E \rightarrow \mathbb{R}$ is in $\mathscr{G}^{0,1}([0, T] \times E)$ if $f$ is continuous and Gâteaux differentiable with respect to every $x \in E$ and the gradient $\nabla f:[0, T] \times E \rightarrow L(E, \mathbb{R})$ is strongly continuous. For more details on this classes of Gâteaux differentiable functions see [11, Section 2.2].

\subsection{The forward equation}

We are given the Markov process $X$ in $E$ (also denoted $X^{t, x}$ to stress the dependence on the initial conditions) solution to the equation

$$
\left\{\begin{array}{l}
d X_{\tau}^{t, x}=A X_{\tau}^{t, x} d \tau+F\left(X_{\tau}^{t, x}\right) d \tau+(-A)^{-\alpha} d W_{\tau}, \quad \tau \in[t, T] \\
X_{t}^{t, x}=x \in E
\end{array}\right.
$$

where $\left(W_{\tau}\right)_{\tau \in[0, T]}$ is a cylindrical Wiener process with values in $H$, see e.g. [6] for details on cylindrical Wiener processes in infinite dimensions. From now on $\left\{\mathscr{F}_{\tau}, \tau \geq 0\right\}$ will be the natural filtration generated by the Wiener process and augmented in the usual way.

We assume the following on the coefficients of equation (2.1).

Hypothesis 2.1. $\quad$ 1. A is a linear operator which generates a contraction analytic semigroup $\left(e^{t A}\right)_{t \geq 0}$ on the Hilbert space $H$ and there exist $c, \omega>0$ such that $\left|e^{t A} h\right|_{H} \leq c e^{-\omega t}|h|_{H}$ for any $h \in H$ and any $t \geq 0$. Further, the restriction of $A$ to $E$ generates a contraction $C_{0}$ (or analytic) semigroup on $E$.

2. The stochastic convolution

$$
w^{A}(s, t):=\int_{s}^{t} e^{(t-u) A}(-A)^{-\alpha} d W_{u}, \quad 0 \leq s<t \leq T,
$$

admits an E-continuous version, and, for any $p \geq 2, \mathbb{E}\left[\sup _{t \in[0, T]}\left|w^{A}(t)\right|_{E}^{p}\right]<+\infty$ (when $s=0$ we write $w^{A}(t)$ instead of $\left.w^{A}(0, t)\right)$.

3. $F: D(F) \subset H \rightarrow H$ is a measurable and dissipative map, and $E \subseteq D(F)$.

4. The restriction $F_{E}$ of $F$ to $E$ is a map from $E$ to $E$ which is measurable and dissipative (where no confusion is possible, we simply write $F$ instead of $\left.F_{E}\right) . F \in \mathscr{G}^{1}(H, H)$ and $F_{E}$ is Fréchet differentiable. Further, there exist $a, c, \gamma>0, m \in \mathbb{N}$ and for any $z \in E$ an element $z^{*} \in \partial|z|_{E}$, such that, for any $x \in E, h \in H$,

$$
\begin{aligned}
\left|F_{E}(x)\right|_{E} & \leq c\left(1+|x|_{E}^{2 m+1}\right), \\
\|\nabla F(x)\|_{\mathscr{L}(E)} & \leq c\left(1+|x|_{E}^{2 m}\right), \\
\left\langle F(x+z)-F(x), z^{*}\right\rangle_{E \times E^{*}} & \leq-a|z|_{E}^{2 m+1}+c\left(1+|x|_{E}^{\gamma}\right), \\
|\nabla F(x) h|_{H} & \leq c\left(1+|x|_{E}^{2 m}\right)|h|_{H} .
\end{aligned}
$$

5. $\alpha \in(0,1 / 2)$.

By Hypothesis 2.1-1. and the Kuratowski theorem, see e.g. [24], Chapter I, Theorem 3.9, it follows that $E$ is a Borel set in $H$. 
Remark 2.2. Since by Hypothesis 2.1-3.-4. F is differentiable and dissipative, we get

$$
1 \geq|z-\alpha D F(x) z|_{E}, \quad x, z \in E, \quad|z|_{E}=1, \quad \alpha>0 .
$$

In particular, from the Hahn-Banach theorem, there exists $z^{*} \in \partial|z|_{E}$ such that $|z-\alpha D F(x) z|_{E}=$ $\left\langle z-\alpha D F(x) z, z^{*}\right\rangle_{E \times E^{*}}$, and therefore $\left\langle D F(x) z, z^{*}\right\rangle_{E \times E^{*}} \leq 0$. Further, from [6, Appendix D] we have

$$
D_{-}|x|_{E} y=\min \left\{\left\langle y, x^{*}\right\rangle_{E \times E^{*}}: x^{*} \in \partial|x|_{E}\right\} .
$$

Remark 2.3. Since $A$ generates a contraction semigroup on $E$, then $A$ is dissipative, and for any $x \in D(A)$ we have $\left\langle A x, x^{*}\right\rangle_{E \times E^{*}} \leq 0, x^{*} \in \partial|x|_{E}$, see Example D.8 in [6].

We now give an example of spaces $E$ and $H$ and of operator $A$ satisfying Hypothesis 2.1-1.-2.

Example 2.4. Let $d, n \in \mathbb{N}$ with $d \leq 3, \mathscr{O} \subset \mathbb{R}^{d}$ be an open bounded set, $H:=L^{2}\left(\mathscr{O} ; \mathbb{R}^{n}\right)$ and $E:=$ $C\left(\overline{\mathscr{O}} ; \mathbb{R}^{n}\right)$. Further, let $A$ be the realization in $H$ of the operator

$$
\mathscr{A}-(\rho+1) I=(\Delta-(\rho+1) I, \ldots, \Delta-(\rho+1) I),
$$

with boundary conditions $\mathscr{B} u=0$, where $\mathscr{B}=\left(\mathscr{B}_{1}, \ldots, \mathscr{B}_{n}\right)$ and

$$
\mathscr{B}_{k}=I d, \quad \text { or } \quad \mathscr{B}_{k}:=\sum_{i=1}^{d} \nu_{i}(\xi) \frac{\partial}{\partial \xi_{i}}, \quad \xi \in \partial \mathscr{O}, k=1, \ldots, n,
$$

where $\nu_{i}$ is the normal vector to the boundary of $\overline{\mathscr{O}}$. As shown for example in [17], A satisfies Hypothesis 2.1-1. Moreover, [3, Lemma 6.1.2] with $Q=(-A)^{-\alpha}$ shows that Hypothesis 2.1-2. is satisfied with this choice of $H, E$ and $A$.

In the following proposition we collect important results on the solution of the forward equation (2.1). We recall that, given $x \in E$ and $t \in[0, T]$, a mild solution to (2.1) is an adapted process $X^{t, x}:[0, T] \times \Omega \rightarrow E$ which satisfies

$$
X_{\tau}^{t, x}=e^{(\tau-t) A} x+\int_{t}^{\tau} e^{(\tau-s) A} F\left(X_{s}^{t, x}\right) d s+\int_{t}^{\tau} e^{(\tau-s) A} d W_{s}, \quad \tau \in[t, T], \mathbb{P} \text {-a.s. }
$$

Proposition 2.5. Let Hypothesis 2.1 hold true. Then the following hold.

(i) For any $x \in E, t \in[0, T]$, the problem (2.1) admits a unique mild solution $X^{t, x} \in \mathscr{S}^{p}((t, T] ; E)$, for any $p \geq 1$. If $A$ generates a strongly continuous semigroup on $E$, then the process $X^{t, x}$ is also continuous up to $t$. Moreover, there exists a positive constant c such that, for any $\tau \in[t, T]$,

$$
\left|X_{\tau}^{t, x}\right|_{E} \leq e^{c \tau}|x|_{E}+h(t, \tau), \quad \mathbb{P} \text {-a.s. }
$$

where

$$
h(t, \tau):=c e^{c(\tau-t)} \int_{t}^{\tau}\left(1+\left|w^{A}(t, s)\right|_{E}^{2 m+1}\right) d s+\sup _{s \in[t, \tau]}\left|w^{A}(t, s)\right|_{E}
$$

(ii) For any $x \in E, t \in[0, T]$, the mild solution $X^{t, x}$ to (2.1) is Gâteaux differentiable as a map from E to $\mathscr{S}^{p}([t, T] ; E)$, and

$$
\sup _{x \in E, \tau \in[t, T]}\left|\nabla_{x} X_{\tau}^{t, x} z\right|_{E} \leq|z|_{E}, \quad z \in E, \quad \mathbb{P} \text {-a.s. }
$$

Moreover, $X^{t, x}$ is Gâteaux differentiable as a map from $E$ to $\mathscr{S}^{p}([0, T] ; H)$, and

$$
\sup _{x \in E, \tau \in[t, T]}\left|\nabla_{x} X_{\tau}^{t, x} h\right|_{H} \leq|h|_{H}, \quad h \in H, \quad \mathbb{P} \text {-a.s. }
$$


(iii) For any $x \in E, t \in[0, T]$ and $\tau \in[t, T]$,

$$
\nabla_{x} X_{\tau}^{t, x} h=e^{(\tau-t) A} h+\int_{t}^{\tau} e^{(\tau-s) A} \nabla F\left(X_{s}^{t, x}\right) \nabla_{x} X_{s}^{t, x} h d s, \quad h \in H, \quad \mathbb{P} \text {-a.s. }
$$

Proof. Item $(i)$ can be proved arguing as in [6, Theorem 7.13].

The first part of (ii) and inequality (2.5) follow from [19, Propositions $3.10 \& 3.13]$. We claim that

$$
\sup _{x \in E, \tau \in[t, T]}\left|\nabla_{x} X_{\tau}^{t, x} z\right|_{H} \leq|z|_{H}, \quad z \in E \quad \mathbb{P} \text {-a.s. }
$$

If the claim is true, since $E$ is densely embedded into $H$, by approximation we immediately deduce (2.6) for any $h \in H$. In order to prove (2.8), we consider $z \in E$ and the approximating processes $G_{\tau}^{n} z:=n R(n, A) \nabla_{x} X_{\tau}^{t, x} z, n \in \mathbb{N}$, where $R(n, A):=(n I-A)^{-1}$. Then, $G_{\tau}^{n} z$ is a strict solution to

$$
\frac{d}{d t} G_{\tau}^{n} z=A G_{\tau}^{n} z+\nabla F\left(X_{\tau}^{t, x}\right) G_{\tau}^{n} z, \quad \tau \in(t, T], \quad G_{t}^{n} z=n R(n, A) z .
$$

The dissipativity of $F$ and $A$ implies $\frac{d}{d \tau}\left|G_{\tau}^{n} z\right|_{H}^{2} \leq 0$, which gives $\left|G_{\tau}^{n} z\right|_{H} \leq|n R(n, A) z|_{H}$. Letting $n \rightarrow+\infty$ we get $(2.8)$.

It remains to prove (iii). To this end, we recall that (see e.g. [19]), for any $x, z \in E$, the process $\nabla_{x} X_{\tau}^{t, x} z$ is a mild solution to

$$
\left\{\begin{array}{l}
d \zeta_{\tau}=A \zeta_{\tau} d \tau+\nabla F\left(X_{\tau}^{t, x}\right) \zeta_{\tau}, \quad \tau \in[t, T] \\
\zeta_{t}=z \in E
\end{array}\right.
$$

and therefore

$$
\nabla_{x} X_{\tau}^{t, x} z=e^{(\tau-t) A} z+\int_{t}^{\tau} e^{(\tau-s) A} \nabla F\left(X_{s}^{t, x}\right) \nabla_{x} X_{s}^{t, x} z d s, \quad \mathbb{P} \text {-a.s. }
$$

Let $h \in H$ and let $\left(h_{n}\right) \subset E$ be an approximating sequence of $h$ in $H$. If we replace $h_{n}$ to $z$ in (2.10), from (ii) we deduce that the left-hand side of (2.10) and the first term in the right-hand side of $(2.10)$ converge respectively to $\nabla_{x} X_{\tau}^{t, x} h$ and to $e^{(\tau-t) A} h$, as $n \rightarrow+\infty$. As far as the integral in the right-hand side of (2.10) is considered, with $z$ replaced by $h_{n}$, again from $(i i)$ we infer that

$$
e^{(\tau-s) A} \nabla F\left(X_{s}^{t, x}\right) \nabla_{x} X_{s}^{t, x} h_{n} \rightarrow e^{(\tau-s) A} \nabla F\left(X_{s}^{t, x}\right) \nabla_{x} X_{s}^{t, x} h, \quad \mathbb{P} \text {-a.s. },
$$

as $n \rightarrow+\infty$. Thanks to Hypothesis 2.1-4., estimate (2.4) and (2.6), we can apply the dominated convergence theorem and therefore

$$
\int_{t}^{\tau} e^{(\tau-s) A} \nabla F\left(X_{s}^{t, x}\right) \nabla_{x} X_{s}^{t, x} h_{n} \rightarrow \int_{t}^{\tau} e^{(\tau-s) A} \nabla F\left(X_{s}^{t, x}\right) \nabla_{x} X_{s}^{t, x} h, \quad \mathbb{P} \text {-a.s. }
$$

as $n \rightarrow+\infty$, which gives (2.7).

Now we show that for any $x, z \in E$ and any $t \in[0, T]$, the process $\nabla_{x} X^{t, x} z$ belongs to $D(-A)^{1 / 2}$ a.e. in $(t, T)$ and $\mathbb{P}$-a.s., and satisfies useful estimates.

Proposition 2.6. Let Hypothesis 2.1 holds true, and let $x \in E, z \in H$ and $t \in[0, T]$. Then, $\nabla_{x} X^{t, x} z \in$ $D\left((-A)^{1 / 2}\right)$, a.e. in $(t, T)$ and $\mathbb{P}$-a.s., and there exists a positive constant $C$ such that, for any $\varepsilon \in[0,1 / 2]$,

$$
\int_{t}^{\tau}\left|(-A)^{\varepsilon} \nabla_{x} X_{s}^{t, x} z\right|_{H}^{2} d s \leq C(\tau-t)^{1-2 \varepsilon}|z|_{H}^{2}, \quad \tau \in[t, T], \quad \mathbb{P} \text {-a.s. }
$$


Proof. Let $x \in E$. We prove (2.11) for $t=0$, the case $t \in[0, T]$ can be proved by analogous computations. We first assume that $z \in E$. Let $\nabla_{x} X_{t}^{x} z$ be a strict solution to (2.9), otherwise we can approximate it by smooth processes, as in the proof of Proposition 2.5, item (ii). The dissipativity of $F$ in $H$ gives

$$
\frac{d}{d s}\left|\nabla_{x} X_{s}^{x} z\right|_{H}^{2}=\left\langle A \nabla_{x} X_{s}^{x} z, \nabla_{x} X_{s}^{x} z\right\rangle_{H}+\left\langle\nabla F\left(X_{s}^{x}\right) \nabla_{x} X_{s}^{x} z, \nabla_{x} X_{s}^{x} z\right\rangle_{H} \leq\left\langle A \nabla_{x} X_{s}^{x} z, \nabla_{x} X_{s}^{x} z\right\rangle_{H},
$$

for any $s \in[0, T]$. Integrating between 0 and $\tau \in[0, T]$ we get

$$
\left|\nabla_{x} X_{\tau}^{x} z\right|_{H}^{2}+\int_{0}^{\tau}\left\langle-A \nabla_{x} X_{s}^{x} z, \nabla_{x} X_{s}^{x} z\right\rangle_{H} d s \leq|z|_{H}^{2}
$$

Since $\left\langle-A \nabla_{x} X_{s}^{x} z, \nabla_{x} X_{s}^{x} z\right\rangle_{H}=\left|(-A)^{1 / 2} \nabla_{x} X_{s}^{x} z\right|_{H}^{2}$ for any $s \in[0, t]$, from (2.6) we deduce that $\nabla_{x} X_{\tau}^{x} z \in$ $D\left((-A)^{1 / 2}\right)$ for any $\tau \in[0, T]$. Thus (2.11) holds for $\varepsilon=1 / 2, t=0$ and any $z \in E$.

Let us now consider $\varepsilon \in[0,1 / 2)$. From interpolation estimates (see e.g. [17, Section 2.2])

$$
\left|(-A)^{\varepsilon} e^{t A} x\right|_{H} \leq C_{\varepsilon}|x|_{H}^{1-2 \varepsilon}\left|(-A)^{1 / 2} x\right|_{H}^{2 \varepsilon}, \quad x \in D\left((-A)^{1 / 2}\right) .
$$

By replacing $x$ by $\nabla_{x} X_{s}^{x} z$ in $(2.12)$, we get

$$
\begin{aligned}
\int_{0}^{\tau}\left|(-A)^{\varepsilon} \nabla_{x} X_{s}^{x} z\right|_{H}^{2} d s & \leq C_{\varepsilon}^{2} \int_{0}^{\tau}\left|(-A)^{1 / 2} \nabla_{x} X_{s}^{x} z\right|_{H}^{4 \varepsilon}\left|\nabla_{x} X_{s}^{x} z\right|_{H}^{2-4 \varepsilon} d s \\
& \leq C_{\varepsilon}^{2}\left(\int_{0}^{\tau}\left|(-A)^{1 / 2} \nabla_{x} X_{s}^{x} z\right|_{H}^{2} d s\right)^{2 \varepsilon}\left(\int_{0}^{\tau}\left|\nabla_{x} X_{s}^{x} z\right|_{H}^{2} d s\right)^{1-2 \varepsilon} \leq C \tau^{1-2 \varepsilon}|z|_{H}^{2}
\end{aligned}
$$

with $C:=\sup _{\varepsilon \in(0,1 / 2)} C_{\varepsilon}^{2}$. We conclude that (2.11) holds for $t=0, \varepsilon \in[0,1 / 2]$ and any $z \in E$.

Let us now consider $z \in H$, and let $\left(z_{n}\right) \subset E$ be an approximating sequence of $z$ in $H$. Then, from (2.6), for any $\tau \in[0, T]$ we get

$$
\nabla_{x} X_{\tau}^{x} z_{n} \rightarrow \nabla_{x} X_{\tau}^{x} z \quad \mathbb{P} \text {-a.s. in } H, \text { as } n \rightarrow+\infty \text {. }
$$

Since (2.11) holds for any $z \in E$, it follows that $\left((-A)^{1 / 2} \nabla_{x} X^{x} z_{n}\right)$ is a Cauchy sequence in $\mathscr{M}^{2}([0, T] ; H)$, and therefore there exists a process $\xi \in \mathscr{M}^{2}([0, T] ; H)$ such that $(-A)^{1 / 2} \nabla_{x} X^{x} z_{n} \rightarrow \xi$ in $\mathscr{M}^{2}([0, T] ; H)$. Since $(-A)^{-1 / 2}$ is a bounded operator on $H$, it follows that

$$
\nabla_{x} X^{x} z_{n}=(-A)^{-1 / 2}(-A)^{1 / 2} \nabla_{x} X^{x} z_{n} \rightarrow(-A)^{-1 / 2} \xi
$$

in $\mathscr{M}^{2}([0, T] ; H)$. Therefore, also by $(2.13),(-A)^{-1 / 2} \xi=\nabla_{x} X^{x} z$ a.e. in $(0, T)$ and $\mathbb{P}$-a.s., which means that $\nabla_{x} X^{x} z \in D\left((-A)^{1 / 2}\right)$ a.e. in $(0, T)$ and $\mathbb{P}$-a.s., and $(-A)^{1 / 2} \nabla_{x} X^{x} z=\xi$ a.e. in $(0, T)$ and $\mathbb{P}$-a.s. In particular, we get

$$
\int_{0}^{t}\left|(-A)^{1 / 2} \nabla_{x} X_{s}^{t, x} z\right|_{H}^{2} d s \leq C|z|_{H}^{2} .
$$

Again, by applying interpolation estimates we see that (2.11) holds for $\varepsilon \in[0,1 / 2], t=0$ and any $z \in H$.

We end this section by giving pointwise estimates of $(-A)^{\alpha} \nabla_{x} X_{\tau}^{t, x} z$. In particular, we improve the result of Proposition 2.6, by obtaining that $\nabla_{x} X_{\tau}^{t, x}$ belongs to $D\left((-A)^{\alpha}\right)$ for any $\tau \in[t, T]$, P-a.s.

Proposition 2.7. Let Hypothesis 2.1 holds true and let $x \in E, z \in H$ and $t \in[0, T]$. Then, for any $x \in E$ and $z \in H$,

$$
\mathbb{E}\left[\sup _{\tau \in[t, T]}\left|(-A)^{\alpha} \nabla_{x} X_{\tau}^{t, x} z\right|_{H}\right] \leq C|z|_{H}\left((\tau-t)^{-\alpha}+(\tau-t)^{1-\alpha}\left(|x|_{E}^{2 m+1}+C_{T}\right)\right)
$$


and if in addition $z \in D\left((-A)^{\alpha}\right)$, then (2.17) gives

$$
\mathbb{E}\left[\sup _{\tau \in[t, T]}\left|(-A)^{\alpha} \nabla_{x} X_{\tau}^{t, x} z\right|_{H}\right] \leq C\left(\left|(-A)^{\alpha} z\right|_{H}+(\tau-t)^{1-\alpha}\left(|x|_{E}^{2 m+1}+C_{T}\right)|z|_{H}\right),
$$

where $C_{T}:=\mathbb{E}\left[\sup _{\tau \in[0, T]}\left|w^{A}(\tau)\right|_{E}^{2 m+1}\right]$.

Proof. We prove estimate (2.16), then (2.17) follows from analogous arguments. Fix $x \in E, z \in H$ and let us consider $t=0$. We recall that $A$ generates an analytic semigroup on $H$ and therefore $e^{t A} x$ belongs to $D\left((-A)^{k}\right)$ for any $k \in \mathbb{N}$ and any $h \in H$, and $\left|(-A)^{\beta} e^{t A} h\right|_{H} \leq C_{\beta} t^{-\beta}|h|_{H}$ for any $\beta \geq 0$ and some positive constant $C_{\beta}$. This means that $\nabla_{x} X_{\tau}^{x} z \in D\left((-A)^{\alpha}\right)$ for any $\tau \in[0, T]$ and, recalling (2.7),

$$
(-A)^{\alpha} \nabla_{x} X_{\tau}^{x} z=(-A)^{\alpha} e^{\tau A} z+\int_{0}^{\tau}(-A)^{\alpha} e^{(\tau-s) A} \nabla_{x} F\left(X_{s}^{x}\right) \nabla_{x} X_{s}^{x} z d x, \quad \mathbb{P} \text {-a.s. }
$$

From Hypothesis 2.1-4. and (2.6) we deduce that

$$
\begin{aligned}
\left|(-A)^{\alpha} \nabla_{x} X_{\tau}^{x} z\right|_{H} & \leq C_{\alpha} \tau^{-\alpha}|z|_{H}+c C_{\alpha} \int_{0}^{\tau}(\tau-s)^{-\alpha}\left|\nabla F\left(X_{s}^{x}\right) \nabla_{x} X_{s}^{x} z\right|_{H} d s \\
& \leq C|z|_{H}\left(\tau^{-\alpha}+\tau^{1-\alpha}\left(|x|_{E}^{2 m+1}+\sup _{\tau \in[0, T]}\left|w^{A}(\tau)\right|_{E}^{2 m+1}\right)\right), \quad \mathbb{P} \text {-a.s., }
\end{aligned}
$$

for some positive constant $C$ independent of $x, z$. Then, for any $\tau \in(t, T]$,

$$
\left|(-A)^{\alpha} \nabla_{x} X_{\tau}^{t, x} z\right|_{H} \leq C|z|_{H}\left((\tau-t)^{-\alpha}+(\tau-t)^{1-\alpha}\left(|x|_{E}^{2 m+1}+\sup _{\tau \in[t, T]}\left|w^{A}(\tau)\right|_{E}^{2 m+1}\right)\right), \mathbb{P} \text {-a.s. }
$$

for some positive constant $C$ independent of $x, z, t$. Further, if $z \in D\left((-A)^{\alpha}\right)$, then

$$
\left|(-A)^{\alpha} \nabla_{x} X_{\tau}^{t, x} z\right|_{H} \leq C\left(\left|(-A)^{\alpha} z\right|_{H}+(\tau-t)^{1-\alpha}\left(|x|_{E}^{2 m+1}+\sup _{\tau \in[t, T]}\left|w^{A}(\tau)\right|_{E}^{2 m+1}\right)|z|_{H}\right), \mathbb{P} \text {-a.s. }
$$

Taking the expectation in (2.16) and (2.17) we get respectively (2.14) and (2.15).

\section{The forward-backward system}

We consider the following forward-backward system of stochastic differential equations (FBSDE for short) for the unknown $(X, Y, Z)$ (also denoted by $\left(X^{t, x}, Y^{t, x}, Z^{t, x}\right)$ to stress the dependence on the initial conditions $t$ and $x)$ : for given $t \in[0, T]$ and $x \in E$,

$$
\begin{cases}d X_{\tau}=A X_{\tau} d \tau+F\left(X_{\tau}\right) d \tau+(-A)^{-\alpha} d W_{\tau}, & \tau \in[t, T], \\ X_{t}=x, & \tau \in[t, T], \\ d Y_{\tau}=-\psi\left(\tau, X_{\tau}, Y_{\tau}, Z_{\tau}\right) d \tau+Z_{\tau} d W_{\tau}, & \\ Y_{T}=\phi\left(X_{T}\right) . & \end{cases}
$$

The second equation is of backward type for the unknown $(Y, Z)$ and depends on the Markov process $X$. Under suitable assumptions on the coefficients $\psi$ (the so-called generator of the BSDE) and $\phi$ we look for a solution consisting of a pair of processes $(Y, Z) \in \mathscr{S}^{2}([t, T]) \times \mathscr{M}^{2}([t, T] ; H)$. More precisely, we will assume that $\psi$ is Lipschitz continuous with respect to $y$ and locally Lipschitz continuous and with quadratic growth with respect to $z$, as stated below.

Hypothesis 3.1. The functions $\phi: E \rightarrow \mathbb{R}$ and $\psi:[0, T] \times E \times \mathbb{R} \times H \rightarrow \mathbb{R}$ in (3.1) satisfy the following. 
(i) $\phi$ is continuous, and there exists a nonnegative constant $K_{\phi}$ such that $|\phi(x)| \leq K_{\phi}$ for every $x \in E$.

(ii) $\psi$ is measurable and, for every fixed $t \in[0, T]$, the map $\psi(t, \cdot, \cdot, \cdot): E \times \mathbb{R} \times H \rightarrow \mathbb{R}$ is continuous. Moreover, there exist nonnegative constants $L_{\psi}$ and $K_{\psi}$ such that

$$
\begin{aligned}
& \left|\psi\left(t, x_{1}, y_{1}, z_{1}\right)-\psi\left(t, x_{2}, y_{2}, z_{2}\right)\right| \leq L_{\psi}\left(\left|x_{1}-x_{2}\right|_{E}+\left|y_{1}-y_{2}\right|+\left|z_{1}-z_{2}\right|_{H}\left(1+\left|z_{1}\right|_{H}+\left|z_{2}\right|_{H}\right)\right), \\
& |\psi(t, x, 0,0)| \leq K_{\psi},
\end{aligned}
$$

for every $t \in[0, T], x_{1}, x_{2} \in E, y_{1}, y_{2} \in \mathbb{R}$ and $z_{1}, z_{2} \in H$.

Theorem 3.2. Assume that Hypotheses 2.1 and 3.1 hold true, and for any $(t, x) \in[0, T] \times E$, let $\left(X^{t, x}, Y^{t, x}, Z^{t, x}\right)$ be a solution to the FBSDE (3.1). Then, there exists a unique solution of the Markovian $B S D E$ in (3.1) such that

$$
\left\|Y^{t, x}\right\|_{\mathscr{S}^{2}([t, T])}+\left\|Z^{t, x}\right\|_{\mathscr{M}^{2}([t, T] ; H)} \leq C,
$$

where $C$ is a constant that may depend on $T, A, F, K_{\psi}, L_{\psi}, K_{\phi}$. Moreover, setting $v(t, x):=Y_{t}^{t, x}$,

$$
Y_{s}^{t, x}=v\left(s, X_{s}^{t, x}\right), \quad \mathbb{P} \text {-a.s., } s \in[t, T],
$$

and there exists a Borel function $u:[t, T] \times E \rightarrow H$ such that

$$
Z_{s}^{t, x}=u\left(s, X_{s}^{t, x}\right), \quad \mathbb{P} \text {-a.s., a.e. } s \in[t, T] .
$$

Proof. The first part of the result substantially follows from [16]. Identities (3.2)-(3.3) are a consequence of the Markov property of $X$, see for instance Theorem 4.1 in [8] or the proof of Theorem 5.1 in [12].

We recall some further estimates for the solution $(Y, Z)$ of the forward-backward system (3.1). In particular, $Z \in \mathscr{M}^{p}([t, T] ; H)$, for any $p \geq 1$. The corresponding proof can be found e.g. in [21].

Proposition 3.3. Assume that Hypotheses 2.1 and 3.1 hold true, and for any $(t, x) \in[0, T] \times E$, let $\left(X^{t, x}, Y^{t, x}, Z^{t, x}\right)$ be a solution to the FBSDE (3.1). Then, for all $p \geq 1$,

$$
\left\|Y^{t, x}\right\|_{\mathscr{S}^{p}([t, T])}+\left\|Z^{t, x}\right\|_{\mathscr{M}^{p}([t, T] ; H)} \leq C,
$$

where $C$ is a constant that may depend on $T, A, F, K_{\psi}, L_{\psi}, K_{\phi}$.

At this point, we aim at proving a stability result for the BSDE when the final datum and the generator are approximated by sequences of Fréchet differentiable functions $\left(\phi_{n}\right)_{n \geq 1},\left(\psi_{\ell}\right)_{\ell \geq 1}$, converging pointwise respectively to $\phi$ and $\psi$, and such that, for all $t \in[0, T], x, x_{1}, x_{2} \in E, y_{1}, y_{2} \in \mathbb{R}, z_{1}, z_{2} \in H$,

$$
\begin{aligned}
& \left|\phi_{n}(x)\right| \leq K_{\phi}, \quad\left|\psi_{\ell}(t, x, 0,0)\right| \leq K_{\psi}, \\
& \left|\psi_{\ell}\left(t, x_{1}, y_{1}, z_{1}\right)-\psi_{\ell}\left(t, x_{2}, y_{2}, z_{2}\right)\right| \leq L_{\psi}\left(\left|x_{1}-x_{2}\right|_{E}+\left|y_{1}-y_{2}\right|+\left|z_{1}-z_{2}\right|_{H}\left(1+\left|z_{1}\right|_{H}+\left|z_{2}\right|_{H}\right)\right) .
\end{aligned}
$$

To provide such approximations we extend the result in [25] valid for Hilbert spaces: by using Schauder basis, the approximation performed in that paper can be achieved also in Banach spaces, along the lines of what is done in [18]. We start by introducing the following objects.

Definition 3.4. $\quad$ i) Denote by $\left(e_{n}\right)_{n \geq 1}$ the normalized Schauder basis in $E$ and by $\left(h_{n}\right)$ an orthonormal basis of $H$. For any $n \in \mathbb{N}$, we define the projections $Q_{n}: H \rightarrow \mathbb{R}^{n}$ and $P_{n}: E \rightarrow \mathbb{R}^{n}$ as follows:

$$
Q_{n} z:=\left(z_{1}, \ldots, z_{n}\right), \quad P_{n} x:=\left(x_{1}, \ldots, x_{n}\right),
$$

for any $z \in H$ and $x \in E$ with $z=\sum_{n=1}^{\infty} z_{n} h_{n}$ and $x=\sum_{n=1}^{\infty} x_{n} e_{n}, z_{n}, x_{n} \in \mathbb{R}$. 
ii) We consider nonnegative smooth kernels $\vartheta \in C_{c}^{\infty}(\mathbb{R})$ and $\rho_{m} \in C_{c}^{\infty}\left(\mathbb{R}^{m}\right), m \in \mathbb{N}$, such that $\operatorname{supp}(\vartheta) \subseteq\{\zeta \in \mathbb{R}:|\zeta| \leq 1\}, \quad \operatorname{supp}\left(\rho_{m}\right) \subseteq\left\{\xi \in \mathbb{R}^{m}:|\xi| \leq m^{-1}\right\}, \quad\|\vartheta\|_{L^{1}(\mathbb{R})}=\left\|\rho_{m^{m}}\right\|_{L^{1}\left(\mathbb{R}^{m}\right)}=1$.

iii) For any $n, \ell \in \mathbb{N}$, we set $\vartheta_{\ell}(\zeta)=\ell \vartheta(\ell \zeta)$ for any $\zeta \in \mathbb{R}$, and

$$
\begin{aligned}
& \phi_{n}(x)=\int_{\mathbb{R}^{n}} \rho_{n}\left(\xi-P_{n} x\right) \phi\left(\sum_{i=1}^{n} \xi_{i} e_{i}\right) d \xi \\
& \psi_{\ell}(t, x, y, z):=\int_{\mathbb{R}^{\ell}} \int_{\mathbb{R}^{\ell}} \int_{\mathbb{R}} \rho_{\ell}\left(\xi-P_{\ell} x\right) \rho_{\ell}\left(\eta-Q_{\ell} z\right) \vartheta_{\ell}(y-\zeta) \psi\left(t, \sum_{i=1}^{\ell} \xi_{i} e_{i}, \zeta, \sum_{j=1}^{\ell} \eta_{j} h_{j}\right) d \zeta d \eta d \xi
\end{aligned}
$$

It is not hard to prove the following lemma.

Lemma 3.5. Le $\phi$ and $\psi$ satisfy Hypothesis 3.1. Then the following hold.

(i) For any $n \in \mathbb{N}$, the function $\phi_{n}$ in (3.6) is Fréchet differentiable, satisfies estimate (3.4), and

$$
\lim _{n \rightarrow+\infty} \phi_{n}(x)=\phi(x), \quad x \in E .
$$

(ii) For any $\ell \in \mathbb{N}$, the function $\psi_{\ell}$ in (3.7) is Fréchet differentiable with respect to $x, y$, $z$, satisfies estimates (3.4)-(3.5), and

$$
\lim _{\ell \rightarrow+\infty} \psi_{\ell}(t, x, y, z)=\psi(t, x, y, z), \quad(t, x, y, z) \in[0, T] \times E \times \mathbb{R} \times H .
$$

We can now give a stability result for the Markovian BSDE in (3.1) related to a forward process $X$ taking values in the Banach space $E$, when the final datum and the generator are approximated respectively by the sequences $\left(\phi_{n}\right)_{n \geq 1}$ and $\left(\psi_{\ell}\right)_{\ell \geq 1}$. Notice that a similar result is proved in [21], where the forward process $X$ takes its values in a Hilbert space $H$ : there the final datum and the generator are approximated in the norm of the uniform convergence by means of their inf-sup convolutions. Clearly, the following result holds true if we approximate only $\psi$ or $\phi$.

Proposition 3.6. Assume that Hypotheses 2.1 and 3.1 hold true. For any $(t, x) \in[0, T] \times E$, let $(X, Y, Z)$ be a solution to the FBSDE (3.1). Let $\left(Y^{n, l}, Z^{n, l}\right)$ be the solution of the BSDE in the forward-backward system

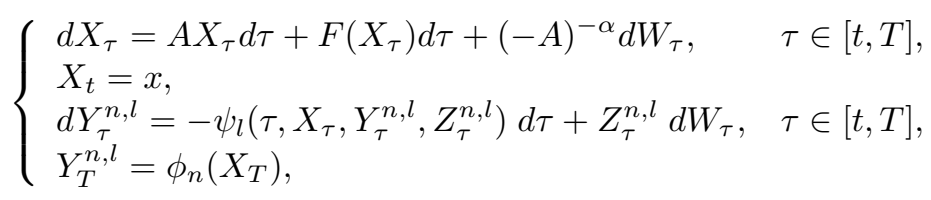

that is, the FBSDE (3.1) with final datum equal to $\phi_{n}$ in (3.6) in place of $\phi$, and with generator $\psi_{l}$ in (3.7) in place of $\psi$. Then, for all $p \geq 1$, the unique solution of the Markovian BSDE in (3.1) is such that

$$
\left\|Y-Y^{n, l}\right\|_{\mathscr{S}^{p}([t, T])}+\left\|Z-Z^{n, l}\right\|_{\mathscr{M}^{p}([t, T] ; H)} \rightarrow 0 \quad \text { as } n, l \rightarrow \infty .
$$

Proof. Thanks to (3.4), (3.5) and to Proposition 3.3, the pair of processes $\left(Y^{n, l}, Z^{n, l}\right)$ is bounded in $\mathscr{S}^{p}([t, T]) \times \mathscr{M}^{p}([t, T] ; H)$, uniformly with respect to $n, l$. The BSDE satisfied by the pair of the difference processes $\left(Y^{n, l}-Y, Z^{n, l}-Z\right)$ is

$$
\left\{\begin{array}{l}
d\left(Y_{\tau}^{n, l}-Y_{\tau}\right)=\left(\psi\left(\tau, X_{\tau}, Y_{\tau}, Z_{\tau}\right)-\psi_{l}\left(\tau, X_{\tau}, Y_{\tau}^{n, l}, Z_{\tau}^{n, l}\right)\right) d \tau+\left(Z_{\tau}^{n, l}-Z_{\tau}\right) d W_{\tau}, \quad \tau \in[t, T] \\
Y_{T}^{n, l}-Y_{T}=\phi_{n}\left(X_{T}\right)-\phi\left(X_{T}\right) .
\end{array}\right.
$$


Writing the previous equation in the integral form, we get

$$
\begin{aligned}
& Y_{\tau}^{n, l}-Y_{\tau} \\
& =\phi_{n}\left(X_{T}\right)-\phi\left(X_{T}\right)-\int_{\tau}^{T}\left(Z_{s}^{n, l}-Z_{s}\right) d W_{s}+\int_{\tau}^{T}\left(\psi\left(s, X_{s}, Y_{s}, Z_{s}\right)-\psi_{l}\left(s, X_{s}, Y_{s}, Z_{s}\right)\right) d s \\
& +\int_{\tau}^{T}\left(\psi_{l}\left(s, X_{s}, Y_{s}, Z_{s}\right)-\psi_{l}\left(s, X_{s}, Y_{s}, Z_{s}^{n, l}\right)\right) d s \\
& +\int_{\tau}^{T}\left(\psi_{l}\left(s, X_{s}, Y_{s}, Z_{s}^{n, l}\right)-\psi_{l}\left(s, X_{s}, Y_{s}^{n, l}, Z_{s}^{n, l}\right)\right) d s \\
= & \phi_{n}\left(X_{T}\right)-\phi\left(X_{T}\right)+\int_{\tau}^{T}\left(\psi\left(s, X_{s}, Y_{s}, Z_{s}\right)-\psi_{l}\left(s, X_{s}, Y_{s}, Z_{s}\right)\right) d s \\
& +\int_{\tau}^{T} \frac{\psi_{l}\left(s, X_{s}, Y_{s}, Z_{s}\right)-\psi_{l}\left(s, X_{s}, Y_{s}, Z_{s}^{n, l}\right)}{Z_{s}-Z_{s}^{n, l}}\left(Z_{s}-Z_{s}^{n, l}\right) d s-\int_{\tau}^{T}\left(Z_{s}^{n, l}-Z_{s}\right) d W_{s} \\
& +\int_{\tau}^{T} \frac{\psi_{l}\left(s, X_{s}, Y_{s}, Z_{s}^{n, l}\right)-\psi_{l}\left(s, X_{s}, Y_{s}^{n, l}, Z_{s}^{n, l}\right)}{Y_{s}-Y_{s}^{n, l}}\left(Y_{s}-Y_{s}^{n, l}\right) d s \\
= & \phi_{n}\left(X_{T}\right)-\phi\left(X_{T}\right)+\int_{\tau}^{T}\left(\psi\left(s, X_{s}, Y_{s}, Z_{s}\right)-\psi_{l}\left(s, X_{\tau}, Y_{s}, Z_{s}\right)\right) d s-\int_{\tau}^{T}\left(Z_{s}^{n, l}-Z_{s}\right) d W_{s}^{n, l} \\
& +\int_{\tau}^{T} \frac{\psi_{l}\left(s, X_{s}, Y_{s}, Z_{s}^{n, l}\right)-\psi_{l}\left(s, X_{s}, Y_{s}^{n, l}, Z_{s}^{n, l}\right)}{Y_{s}-Y_{s}^{n, l}}\left(Y_{s}-Y_{s}^{n, l}\right) d s,
\end{aligned}
$$

where in the last passage we have used that

$$
W_{\tau}^{n, l}=W_{\tau}-\int_{t}^{\tau} \frac{\psi_{l}\left(s, X_{s}, Y_{s}, Z_{s}\right)-\psi_{l}\left(s, X_{s}, Y_{s}, Z_{s}^{n, l}\right)}{Z_{s}-Z_{s}^{n, l}} d s, \quad \tau \geq t,
$$

which, by the Girsanov Theorem (see, e.g., [6, Theorem 10.14]), is a cylindrical Wiener process under an

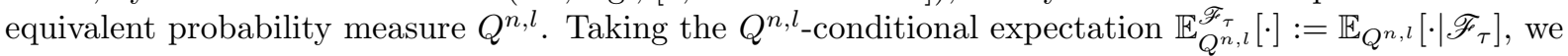
get

$$
\begin{aligned}
Y_{\tau}^{n, l}-Y_{\tau}= & \mathbb{E}_{Q^{n, l}}^{\mathscr{F}_{\tau}}\left[\phi_{n}\left(X_{T}\right)-\phi\left(X_{T}\right)\right]+\mathbb{E}_{Q^{n, l}}^{\mathscr{F}_{\tau}}\left[\int_{\tau}^{T}\left(\psi\left(s, X_{s}, Y_{s}, Z_{s}\right)-\psi_{l}\left(s, X_{s}, Y_{s}, Z_{s}\right)\right) d s\right] \\
& +\mathbb{E}_{Q^{n, l}}^{\mathscr{F}_{\tau}}\left[\int_{\tau}^{T} \frac{\psi_{l}\left(s, X_{s}, Y_{s}, Z_{s}^{n, l}\right)-\psi_{l}\left(s, X_{s}, Y_{s}^{n, l}, Z_{s}^{n, l}\right)}{Y_{s}-Y_{s}^{n, l}}\left(Y_{s}-Y_{s}^{n, l}\right) d s\right] .
\end{aligned}
$$

By taking the absolute value, the expectation and by applying the Gronwall lemma, we deduce that, for all $p \geq 1, Y^{n, l} \rightarrow Y$ in $\mathscr{S}^{p}([t, T])$ as $n, \ell \rightarrow \infty$, with respect to the probability measure $Q^{n, l}$ and also with respect to the original probability measure.

For what concerns the estimate of $Z-Z^{n, l}$, by applying the Itô formula to $\left|Y^{n, l}-Y\right|^{2}$ we get

$$
\begin{aligned}
& \mathbb{E}\left[\left|Y_{t}^{n, l}-Y_{t}\right|^{2}\right]+\mathbb{E}\left[\int_{t}^{T}\left|Z_{\tau}^{n, l}-Z_{\tau}\right|_{H}^{2} d \tau\right] \\
& =\mathbb{E}\left[\left|\phi_{n}\left(X_{T}\right)-\phi\left(X_{T}\right)\right|^{2}\right]-2 \mathbb{E}\left[\int_{t}^{T}\left(Y_{\tau}^{n, l}-Y_{\tau}\right)\left(\psi\left(\tau, X_{\tau}, Y_{\tau}, Z_{\tau}\right)-\psi_{l}\left(\tau, X_{\tau}, Y_{\tau}^{n, l}, Z_{\tau}^{n, l}\right)\right) d \tau\right] \\
& \leq \mathbb{E}\left[\left|\phi_{n}\left(X_{T}\right)-\phi\left(X_{T}\right)\right|^{2}\right]+2 \mathbb{E}\left[\int_{t}^{T}\left|Y_{\tau}^{n, l}-Y_{\tau}\right|\left|\psi\left(\tau, X_{\tau}, Y_{\tau}, Z_{\tau}\right)-\psi_{l}\left(\tau, X_{\tau}, Y_{\tau}, Z_{\tau}\right)\right| d \tau\right] \\
& +2 \mathbb{E}\left[\int_{t}^{T}\left|Y_{\tau}^{n, l}-Y_{\tau} \| \psi_{l}\left(\tau, X_{\tau}, Y_{\tau}, Z_{\tau}\right)-\psi_{l}\left(\tau, X_{\tau}, Y_{\tau}^{n, l}, Z_{\tau}^{n, l}\right)\right| d \tau\right]
\end{aligned}
$$




$$
\begin{aligned}
& \leq \mathbb{E}\left[\left|\phi_{n}\left(X_{T}\right)-\phi\left(X_{T}\right)\right|^{2}\right]+2 \mathbb{E}\left[\sup _{\tau \in[t, T]}\left|Y_{\tau}^{n, l}-Y_{\tau}\right| \int_{t}^{T}\left|\psi\left(\tau, X_{\tau}, Y_{\tau}, Z_{\tau}\right)-\psi_{l}\left(\tau, X_{\tau}, Y_{\tau}, Z_{\tau}\right)\right| d \tau\right] \\
& +C \mathbb{E}\left[\sup _{\tau \in[t, T]}\left|Y_{\tau}^{n, l}-Y_{\tau}\right| \int_{t}^{T}\left(1+\left|Y_{\tau}-Y_{\tau}^{n, l}\right|+\left|Z_{\tau}-Z_{\tau}^{n, l}\right|_{H}\left(1+\left|Z_{\tau}\right|_{H}+\left|Z_{\tau}^{n, l}\right|_{H}\right)\right) d \tau\right] .
\end{aligned}
$$

Let us consider the right-hand side of the above inequality. Thanks to estimates (3.4), (3.5) and to the boundedness of $Y$ and $Y^{n, l}$ in $\mathscr{S}^{p}([t, T] ; E)$, the first two terms converge to 0 as $n, l \rightarrow \infty$ by the dominated convergence theorem. For what concerns the third term, by applying Hôlder's inequality with $p, q$ conjugate exponents, we get

$$
\begin{aligned}
& \mathbb{E}\left[\sup _{\tau \in[t, T]}\left|Y_{\tau}^{n, l}-Y_{\tau}\right| \int_{t}^{T}\left(1+\left|Y_{\tau}-Y_{\tau}^{n, l}\right|+\left|Z_{\tau}-Z_{\tau}^{n, l}\right|_{H}\left(1+\left|Z_{\tau}\right|_{H}+\left|Z_{\tau}^{n, l}\right|_{H}\right)\right) d \tau\right] \\
& \leq C\left(\mathbb{E}\left[\sup _{\tau \in[t, T]}\left|Y_{\tau}^{n, l}-Y_{\tau}\right|^{p}\right]\right)^{\frac{1}{p}}\left(\mathbb{E}\left[\left(\int_{t}^{T}\left(1+\left|Z_{\tau}\right|_{H}^{2}+\left|Z_{\tau}^{n, l}\right|_{H}^{2}\right) d \tau\right)^{q}\right]\right)^{\frac{1}{q}} \rightarrow 0
\end{aligned}
$$

as $n, l \rightarrow \infty$. The stability result for $p=2$ follows, and we can pass to the case of general $p$ in a usual way.

We now state a result on differentiability for the solution of a Markovian BSDE with generator with quadratic growth, with respect to the initial datum $x$.

Proposition 3.7. Assume that Hypotheses 2.1 and 3.1 hold true, and for any $(t, x) \in[0, T] \times E$, let $\left(X^{t, x}, Y^{t, x}, Z^{t, x}\right)$ be a solution to the FBSDE (3.1). Assume moreover that $\phi$ is Gâteaux differentiable with bounded derivative, and that $\psi$ is Gâteaux differentiable with respect to $x, y$ and $z$. Then the triple of processes $\left(X^{t, x}, Y^{t, x}, Z^{t, x}\right)$ is Gâteaux differentiable as a map from $E$ with values in $\mathscr{S}^{2}((t, T] ; E) \times$ $\mathscr{S}^{2}([t, T]) \times \mathscr{M}^{2}([t, T] ; H)$ and, for any $h \in E$,

$$
\left\{\begin{aligned}
-d \nabla_{x} Y_{\tau}^{t, x} h= & \nabla_{x} \psi\left(\tau, X_{\tau}^{t, x}, Y_{\tau}^{t, x}, Z_{\tau}^{t, x}\right) \nabla_{x} X_{\tau}^{t, x} h d \tau+\nabla_{y} \psi\left(\tau, X_{\tau}^{t, x}, Y_{\tau}^{t, x}, Z_{\tau}^{t, x}\right) \nabla_{x} Y_{\tau}^{t, x} h d \tau \\
& +\nabla_{z} \psi\left(\tau, X_{\tau}^{t, x}, Y_{\tau}^{t, x}, Z_{\tau}^{t, x}\right) \nabla_{x} Z_{\tau}^{t, x} h d \tau-\nabla_{x} Z_{\tau}^{t, x} h d W_{\tau}, \quad \tau \in[t, T], \\
\nabla_{x} Y_{T}^{t, x} h= & \nabla_{x} \phi\left(X_{T}^{t, x}\right) \nabla_{x} X_{T}^{t, x} h, \\
d \nabla_{x} X_{\tau}^{t, x} h= & A \nabla_{x} X_{\tau}^{t, x} h d \tau+\nabla F\left(X_{\tau}^{t, x}\right) \nabla_{x} X_{\tau}^{t, x} h d \tau, \quad \tau \in[t, T], \\
\nabla_{x} X_{t}^{t, x} h= & h .
\end{aligned}\right.
$$

Moreover, there exists a constant $C$, only dependent on $T, A, F, K_{\psi}, L_{\psi}, K_{\phi}$, such that

$$
\mathbb{E}\left[\sup _{\tau \in[t, T]}\left|\nabla Y_{\tau}^{t, x} h\right|^{2}+\int_{t}^{T}\left|\nabla_{x} Z_{\tau}^{t, x} h\right|_{H}^{2} d \tau\right] \leq C|h|_{H}^{2} .
$$

Proof. In the case of a Markovian BSDE with generator $\psi$ quadratic with respect to $Z$ and related to a forward process taking values in a Hilbert space, the result is given in Theorem 4.5 of [2]. Since in Proposition 2.5 we have proved the differentiability of $X^{t, x}$ with respect to $x \in E$, the same conclusions hold when the forward process takes values in the Banach space $E$, namely

$$
\mathbb{E}\left[\sup _{\tau \in[t, T]}\left|\nabla_{x} Y_{s}^{t, x} h\right|^{2}+\int_{t}^{T}\left|\nabla_{x} Z_{\tau}^{t, x} h\right|_{H}^{2} d \tau\right] \leq C|h|_{E}^{2} .
$$

The stronger estimate (3.10) comes from Proposition 2.5, estimate (2.6). 


\subsection{Identification of $Z$ and a priori estimates on $(Y, Z)$}

We now prove an a priori estimate on $Z^{t, x}$ depending only on the $L^{\infty}$-norm of the final datum. The novelty towards [21] is that we work in a Banach space and the pseudo-inverse of the diffusion operator is the unbounded operator $(-A)^{\alpha}$. In order to get this estimate and also for the subsequent results of the paper, it will be crucial to prove the identification

$$
Z_{t}^{t, x}=\nabla_{x} Y_{t}^{t, x}(-A)^{-\alpha}
$$

which is new in the Banach space framework and in the case of quadratic generator with respect to $z$. We have to make the following assumption:

Hypothesis 3.8. There exists a Banach space $E_{0} \subset D\left((-A)^{\alpha}\right)$ dense in $H$ such that $(-A)^{-\alpha} E_{0} \subset E$ and $(-A)^{-\alpha}: E_{0} \rightarrow E$ is continuous.

Remark 3.9. Notice that if $\mathscr{D} \subset \mathbb{R}^{2}$ is a bounded open domain with smooth boundary, $H=L^{2}(\mathscr{D})$ and $A$ is the Laplace operator in dimension 2 with Dirichlet boundary conditions, then we can take $E_{0}=D\left((-A)^{\frac{1}{2}}\right)$ and all the requirements of Hypothesis 3.8 are verified.

Theorem 3.10. Assume that Hypotheses 2.1, 3.1 and 3.8 hold true, that $\phi$ is Gâteaux differentiable with bounded derivative, and that $\psi$ is Gâteaux differentiable with respect to $x, y$ and $z$. For any $(t, x) \in[0, T] \times$ $E$, let $\left(X^{t, x}, Y^{t, x}, Z^{t, x}\right)$ be the solution to the FBSDE (3.1). Then the triple of processes $\left(X^{t, x}, Y^{t, x}, Z^{t, x}\right)$ is Gâteaux differentiable as a map from $E$ with values in $\mathscr{S}^{2}((t, T] ; E) \times \mathscr{S}^{2}([t, T]) \times \mathscr{M}^{2}([t, T] ; H)$. Moreover, setting $v(t, x)=Y_{t}^{t, x}$, then, $\mathbb{P}-$ a.s.,

$$
\begin{aligned}
Y_{s}^{t, x} & =v\left(s, X_{s}^{t, x}\right), \quad s \in[t, T], \\
Z_{s}^{t, x} h & =\nabla_{x} v\left(s, X_{s}^{t, x}\right) \nabla_{x} X_{s}^{t, x}(-A)^{-\alpha} h, \quad \text { a.e. } s \in[t, T], h \in E_{0} .
\end{aligned}
$$

Proof. The differentiability properties of $\left(X^{t, x}, Y^{t, x}, Z^{t, x}\right)$ and the identification formula (3.11) directly follow respectively from Proposition 3.7 and formula (3.2) in Theorem 3.2.

Let us now prove identification formula (3.12) for $Z$. Since we are in a Banach space framework, we will follow the lines of the proof of Theorem 3.17 in [19]. However, a substantial difference with respect to [19] is that here we deal with a generator $\psi$ with quadratic growth with respect to $z$, instead of Lipschitz continuous. Fix $t \in[0, T]$. By the definition of the function $v$, we can write

$$
v\left(\tau, X_{\tau}^{t, x}\right)+\int_{t}^{\tau} Z_{\sigma}^{t, x} d W_{\sigma}=v(t, x)+\int_{t}^{\tau} \psi_{\sigma} d \sigma, \quad 0 \leq t \leq \tau \leq T
$$

where we have used the notation $\psi_{\sigma}:=\psi\left(\sigma, X_{\sigma}^{t, x}, Y_{\sigma}^{t, x}, Z_{\sigma}^{t, x}\right)$. Notice that towards [19] we do not have $\psi \in \mathscr{M}^{2}([0, T])$, but we only know that $\psi \in L^{p}\left(\Omega, L^{1}(0, T ; \mathbb{R})\right)$ for any $p \geq 2$. As in [19], we define a family $\mathscr{S}$ of predictable processes with real values in the following way:

$$
\begin{aligned}
\mathscr{S}=\{ & \text { predictable processes } \eta \text { : for any } k=0, \ldots, 2^{n}-1, \\
& \eta_{t} 1_{\left[\frac{k T}{2^{n}}, \frac{(k+1) T}{2^{n}}\right)}(t)=\eta^{k}\left(W_{t_{1}}, \ldots, W_{t_{l_{k}}}\right) \text { for } 0 \leq t_{1} \leq \cdots \leq t_{l_{k}} \leq \frac{k T}{2^{n}}, \\
& \left.\eta^{k} \text { bounded functions in } C^{\infty}\left(\mathbb{R}^{l_{k}}, \mathbb{R}\right) \text { with bounded derivatives of all orders }\right\} .
\end{aligned}
$$

We will briefly write $\eta_{t}=\eta_{t}(W$.$) , where by W$. we mean the trajectory of $W$ up to time $t$.

Let us set $\xi_{t}:=\eta_{t} \varsigma$ for $\varsigma \in E_{0}$. From now on we fix $s>t$, and $\delta>0$, small enough such that $s-\delta>t$. We also identify $H$ with its dual $H^{*}$, and we write $\xi$ for $\xi^{*}$. Multiplying both sides of (3.13), with $\tau$ replaced by $s$, by $\int_{s-\delta}^{s} \xi_{\sigma} d W_{\sigma}$ and taking the expectation, we get

$$
\mathbb{E}\left[v\left(s, X_{s}^{t, x}\right) \int_{s-\delta}^{s} \xi_{\sigma} d W_{\sigma}\right]=\mathbb{E}\left[\int_{t}^{s} \psi_{\sigma} d \sigma \int_{s-\delta}^{s} \xi_{\sigma} d W_{\sigma}\right]+\mathbb{E}\left[\int_{t}^{s} Z_{\sigma}^{t, x} d W_{\sigma} \int_{s-\delta}^{s} \xi_{\sigma} d W_{\sigma}\right] .
$$


It is immediate that

$$
\mathbb{E}\left[\int_{t}^{s-\delta} \psi_{\sigma} d \sigma \int_{s-\delta}^{s} \xi_{\sigma} d W_{\sigma}\right]=0, \quad \mathbb{E}\left[\int_{t}^{s} Z_{\sigma}^{t, x} d W_{\sigma} \int_{s-\delta}^{s} \xi_{\sigma} d W_{\sigma}\right]=\mathbb{E}\left[\int_{s-\delta}^{s} Z_{\sigma}^{t, x} \xi_{\sigma} d \sigma\right],
$$

so (3.14) simplifies in

$$
\mathbb{E}\left[v\left(s, X_{s}^{t, x}\right) \int_{s-\delta}^{s} \xi_{\sigma} d W_{\sigma}\right]=\mathbb{E}\left[\int_{s-\delta}^{s} \psi_{\sigma} d \sigma \int_{s-\delta}^{s} \xi_{\sigma} d W_{\sigma}\right]+\mathbb{E}\left[\int_{s-\delta}^{s} Z_{\sigma}^{t, x} \xi_{\sigma} d \sigma\right] .
$$

By dividing both sides of the previous equality by $\delta$ and letting $\delta \rightarrow 0$, we get

$$
\mathbb{E}\left[Z_{s}^{t, x} \xi_{s}\right]=\lim _{\delta \rightarrow 0} \frac{1}{\delta} \mathbb{E}\left[v\left(s, X_{s}^{t, x}\right) \int_{s-\delta}^{s} \xi_{\sigma} d W_{\sigma}\right]-\lim _{\delta \rightarrow 0} \frac{1}{\delta} \mathbb{E}\left[\int_{s-\delta}^{s} \psi_{\sigma} d \sigma \int_{s-\delta}^{s} \xi_{\sigma} d W_{\sigma}\right] .
$$

We will prove that

$$
\begin{aligned}
& \lim _{\delta \rightarrow 0} \frac{1}{\delta} \mathbb{E}\left[v\left(s, X_{s}^{t, x}\right) \int_{s-\delta}^{s} \xi_{\sigma} d W_{\sigma}\right]=\mathbb{E}\left[\nabla_{x}\left(v\left(s, X_{s}^{t, x}\right)\right)(-A)^{-\alpha} \xi_{s}\right], \\
& \lim _{\delta \rightarrow 0} \frac{1}{\delta} \mathbb{E}\left[\int_{s-\delta}^{s} \psi_{\sigma} d \sigma \int_{s-\delta}^{s} \xi_{\sigma} d W_{\sigma}\right]=0 .
\end{aligned}
$$

If (3.16) and (3.17) hold, then, by (3.15), for every $\eta \in \mathscr{S}, \mathbb{E}\left[Z_{\sigma}^{t, x} \varsigma \eta_{s}\right]=\mathbb{E}\left[\nabla v\left(\sigma, X_{\sigma}^{t, x}\right)(-A)^{-\alpha} \varsigma \eta_{s}\right]$ for almost every $\sigma \in[t, T]$. By the arbitrariness of $\eta$, we would have, for almost every $\sigma \in[t, T]$, $Z_{\sigma}^{t, x} \varsigma=\nabla_{x}\left(v\left(\sigma, X_{\sigma}^{t, x}\right)\right)(-A)^{-\alpha} \varsigma, \mathbb{P}$-a.s. for all $\varsigma \in E_{0}$, and the formula (3.12) would follow.

Let us thus show that (3.16) and (3.17) hold true. We start by proving (3.16). One proceeds as in [1], following also [19]. In particular, for $0 \leq t \leq \sigma \leq T$, we define

$$
W_{\sigma}^{\varepsilon}=W_{\sigma}-\varepsilon \int_{t}^{\sigma} \xi_{r}\left(W_{.}^{\varepsilon}\right) d r
$$

where $\xi_{r}\left(W^{\varepsilon}\right)$ depends on the trajectories of $W^{\varepsilon}$ up to time $r$, and the dependence is given by the definition of $\eta$. The process $\left(W_{\sigma}^{\varepsilon}\right)_{\sigma}$ is defined as the solution of (3.18), which is not considered as a stochastic differential equation, as specified in [1, p. 476]. Equation (3.18) can be solved step by step in each interval

$$
\left[\frac{k T}{2^{n}}, \frac{(k+1) T}{2^{n}}\right), \quad k=0, \ldots, 2^{n}-1 .
$$

$\left(W_{\sigma}^{\varepsilon}\right)_{\sigma}$ is well defined for every $0 \leq \sigma \leq T$, see [19] for more details. Moreover, $W_{\sigma}^{\varepsilon}$ is a function of the trajectories of $W$ up to time $\sigma$, that is, $W_{\sigma}^{\varepsilon}=W_{\sigma}^{\varepsilon}(W$.), and we can write

$$
W_{\sigma}^{\varepsilon}=W_{\sigma}-\varepsilon \int_{t}^{\sigma} \xi_{r}\left(W^{\varepsilon}(W .)\right) d r, \quad 0 \leq t \leq \sigma \leq T .
$$

Now we define a probability measure $Q_{\varepsilon}$ such that

$$
\frac{d Q_{\varepsilon}}{d \mathbb{P}}=\exp \left(\varepsilon \int_{t}^{T} \xi_{\sigma}\left(W^{\varepsilon}(W .)\right) d W_{\sigma}-\frac{\varepsilon^{2}}{2} \int_{t}^{T}\left|\xi_{\sigma}\left(W^{\varepsilon}(W .)\right)\right|^{2} d \sigma\right)
$$

By the Girsanov Theorem, under $Q_{\varepsilon}, W_{\sigma}^{\varepsilon}=W_{\sigma}-\varepsilon \int_{t}^{\sigma} \xi_{r}\left(W^{\varepsilon}(W).\right) d r$ is a cylindrical Wiener process in $H$. By this construction of $\left(W_{\sigma}^{\varepsilon}\right)_{\sigma}$, it is also clear that for every $0 \leq \sigma \leq T, W_{\sigma}^{\varepsilon}$ is pathwise differentiable with respect to $\varepsilon$ and $\left.\frac{d}{d \varepsilon}\right|_{\varepsilon=0} W_{\sigma}^{\varepsilon}=-\int_{t}^{\sigma} \xi_{r}(W) d$.$r , see also [1, p. 476].$ 
By (3.13), the random varaible $v\left(s, X_{s}^{t, x}\right)$ is square integrable and

$$
\begin{aligned}
\mathbb{E}\left[v^{2}\left(s, X_{s}^{t, x}\right)\right] & \leq c\left\{1+\mathbb{E}\left[\left(\int_{t}^{s} \xi_{\sigma} d W_{\sigma}\right)^{2}\right]+\mathbb{E}\left[\left|\int_{t}^{s} \psi_{\sigma} d \sigma\right|^{2}\right]\right\} \\
& \leq c\left\{1+\mathbb{E}\left[\int_{t}^{s}\left|\xi_{\sigma}\right|_{H}^{2} d \sigma\right]+\mathbb{E}\left[\left|\int_{t}^{s} \psi_{\sigma} d \sigma\right|^{2}\right]\right\}<\infty .
\end{aligned}
$$

Therefore, by the Cauchy-Schwarz inequality the expectation of $v\left(s, X_{s}^{t, x}\right) \int_{s-\delta}^{s} \xi_{\sigma} d W_{\sigma}$ is well defined. We claim that

$$
\mathbb{E}\left[v\left(s, X_{s}^{t, x}\right) \int_{s-\delta}^{s} \xi_{\sigma} d W_{\sigma}\right]=\frac{d}{d \varepsilon} \mathbb{E}_{\mid \varepsilon=0}\left[v\left(s, X_{s}^{t, x}\right)\right]
$$

As a matter of fact,

$$
\begin{aligned}
& \frac{d}{d \varepsilon}{ }_{\varepsilon=0} \mathbb{E}_{Q_{\varepsilon}}\left[v\left(s, X_{s}^{t, x}\right)\right]=\frac{d}{d \varepsilon}{ }_{\varepsilon=0} \mathbb{E}\left[v\left(s, X_{s}^{t, x}\right) \exp \left(\varepsilon \int_{s-\delta}^{s} \xi_{\sigma} d W_{\sigma}-\frac{\varepsilon^{2}}{2} \int_{s-\delta}^{s}\left\|\xi_{\sigma}\right\|_{H}^{2} d \sigma\right)\right] \\
& =\lim _{\varepsilon \rightarrow 0} \mathbb{E}\left[v\left(s, X_{s}^{t, x}\right) \frac{1}{\varepsilon}\left\{\exp \left(\varepsilon \int_{s-\delta}^{s} \xi_{\sigma} d W_{\sigma}-\frac{\varepsilon^{2}}{2} \int_{s-\delta}^{s}\left|\xi_{\sigma}\right|_{H}^{2} d \sigma\right)-1\right\}\right]=\mathbb{E}\left[v\left(s, X_{s}^{t, x}\right) \int_{s-\delta}^{s} \xi_{\sigma} d W_{\sigma}\right],
\end{aligned}
$$

where in the last passage we have used the dominated convergence theorem being $\xi$ bounded. Now notice that, in $\left(\Omega, \mathscr{F}, Q_{\varepsilon}\right), X^{t, x}$ is a mild solution to the equation

$$
d X_{\tau}^{t, x}=A X_{\tau}^{t, x} d \tau+F\left(X_{\tau}^{t, x}\right) d \tau+(-A)^{-\alpha} \varepsilon \xi_{\tau}\left(W_{.}^{\varepsilon}\right) d \tau+(-A)^{-\alpha} d W_{\tau}^{\varepsilon}, \quad \tau \in[s-\delta, T] .
$$

On the other hand, in $(\Omega, \mathscr{F}, \mathbb{P})$, we consider the process $X^{\varepsilon}$ which is a mild solution to the equation

$$
\left\{\begin{array}{l}
d X_{\tau}^{\varepsilon}=A X_{\tau}^{\varepsilon} d \tau+F\left(X_{\tau}^{\varepsilon}\right) d \tau+(-A)^{-\alpha} \varepsilon \xi_{\tau}(W .) d \tau+(-A)^{-\alpha} d W_{\tau}, \quad \tau \in[s-\delta, T] \\
X_{s-\delta}^{\varepsilon}=X_{s-\delta}^{t, x}
\end{array}\right.
$$

Then the process $X^{t, x}$ under $Q_{\varepsilon}$ and the process $X^{\varepsilon}$ under $\mathbb{P}$ have the same law, so (3.19) yields

$$
\mathbb{E}\left[v\left(s, X_{s}^{t, x}\right) \int_{s-\delta}^{s} \xi_{\sigma} d W_{\sigma}\right]=\frac{d}{d \varepsilon} \mid \varepsilon=0
$$

Let us set $\dot{X}_{\tau}:=\frac{d}{d \varepsilon}{ }_{\varepsilon=0} X_{\tau}^{\varepsilon}$ and $\Delta^{\varepsilon} X_{\tau}=\frac{X_{\tau}^{\varepsilon}-X_{\tau}}{\varepsilon}, \mathbb{P}$-a.s. for any $\tau \in[s-\delta, T]$. Arguing as in [19], one can prove that

$$
\lim _{\varepsilon \rightarrow 0}\left|\Delta^{\varepsilon} X_{\tau}-\dot{X}_{\tau}\right|_{E}=0, \quad \dot{X}_{\tau}=\int_{s-\delta}^{\tau} \nabla_{x} X_{\tau}^{\sigma, X_{\sigma}^{t, x}}(-A)^{-\alpha} \xi_{\sigma} d \sigma, \quad \tau \in[s-\delta, T], \quad \mathbb{P} \text {-a.s. }
$$

Formula (3.21) in turn allows to show that

$$
\frac{d}{d \varepsilon}{ }_{\varepsilon=0} \mathbb{E}\left[v\left(s, X_{s}^{\varepsilon}\right)\right]=\mathbb{E}\left[\nabla_{x} v\left(s, X_{s}^{t, x}\right) \dot{X}_{s}\right]=\mathbb{E}\left[\nabla_{x} v\left(s, X_{s}^{t, x}\right) \int_{s-\delta}^{s} \nabla_{x} X_{s}^{\sigma, X_{\sigma}^{t, x}}(-A)^{-\alpha} \xi_{\sigma} d \sigma\right],
$$

so that formula (3.20) gives

$$
\mathbb{E}\left[v\left(s, X_{s}^{t, x}\right) \int_{s-\delta}^{s} \xi_{\sigma} d W_{\sigma}\right]=\mathbb{E}\left[\nabla_{x} v\left(s, X_{s}^{t, x}\right) \int_{s-\delta}^{s} \nabla_{x} X_{s}^{\sigma, X_{\sigma}^{t, x}}(-A)^{-\alpha} \xi_{\sigma} d \sigma\right] .
$$


By (3.22) we have

$$
\begin{aligned}
& \lim _{\delta \rightarrow 0} \frac{1}{\delta} \mathbb{E}\left[v\left(s, X_{s}^{t, x}\right) \int_{s-\delta}^{s} \xi_{\sigma} d W_{\sigma}\right]=\lim _{\delta \rightarrow 0} \frac{1}{\delta} \mathbb{E}\left[\nabla_{x} v\left(s, X_{s}^{t, x}\right) \int_{s-\delta}^{s} \nabla_{x} X_{s}^{\sigma, X_{\sigma}^{t, x}}(-A)^{-\alpha} \xi_{\sigma} d \sigma\right] \\
& =\mathbb{E}\left[\nabla_{x} v\left(s, X_{s}^{t, x}\right) \nabla_{x} X_{s}^{s, X_{s}^{t, x}}(-A)^{-\alpha} \xi_{s}\right]=\mathbb{E}\left[\nabla_{x}\left(v\left(s, X_{s}^{t, x}\right)\right)(-A)^{-\alpha} \xi_{s}\right]
\end{aligned}
$$

so (3.16) is proved.

It remains to prove (3.17). Recalling identifications (3.2)-(3.3), we have

$$
\begin{aligned}
& \frac{1}{\delta} \mathbb{E}\left[\int_{s-\delta}^{s} \psi\left(\sigma, X_{\sigma}^{t, x}, Y_{\sigma}^{t, x}, Z_{\sigma}^{t, x}\right) d \sigma \int_{s-\delta}^{s} \xi_{\sigma} d W_{\sigma}\right] \\
& =\frac{1}{\delta} \mathbb{E}\left[\int_{s-\delta}^{s} \psi\left(\sigma, X_{\sigma}, v\left(\sigma, X_{\sigma}\right), u\left(\sigma, X_{\sigma}\right)\right) d \sigma \int_{s-\delta}^{s} \xi_{\sigma} d W_{\sigma}\right] \\
& =\frac{1}{\delta} \frac{d}{d \varepsilon}{ }_{\mid \varepsilon=0} \mathbb{E}\left[\int_{s-\delta}^{s} \psi\left(\sigma, X_{\sigma}^{\varepsilon}, v\left(\sigma, X_{\sigma}^{\varepsilon}\right), u\left(\sigma, X_{\sigma}^{\varepsilon}\right)\right) d \sigma\right]
\end{aligned}
$$

which is the analogous of formula (3.20) with $f_{\delta}:=\int_{s-\delta}^{\cdot} \psi_{\sigma} d \sigma$ in place of $v$. Now we notice that

$$
\frac{1}{\delta} \frac{d}{d \varepsilon}{ }_{\mid \varepsilon=0} \psi\left(\sigma, X_{\sigma}^{\varepsilon}, v\left(\sigma, X_{\sigma}^{\varepsilon}\right), u\left(\sigma, X_{\sigma}^{\varepsilon}\right)\right)=\nabla_{x} \psi_{\sigma} \frac{\dot{X}_{\sigma}}{\delta}+\nabla_{y} \psi_{\sigma} \frac{\dot{Y}_{\sigma}}{\delta}+\nabla_{z} \psi_{\sigma} \frac{\dot{Z}_{\sigma}}{\delta}, \quad \sigma \in[s-\delta, T]
$$

where we have used the notation

$$
(\dot{Y}, \dot{Z}):=\left(\frac{d}{d \varepsilon}_{\mid \varepsilon=0} Y, \frac{d}{d \varepsilon}_{\mid \varepsilon=0} Z\right)=\left(\nabla_{x} v\left(\sigma, X_{\sigma}^{\varepsilon}\right) \dot{X}, \nabla_{x} u\left(\sigma, X_{\sigma}^{\varepsilon}\right) \dot{X}\right) .
$$

By (3.21) and (2.5), we have

$$
\frac{\left|\dot{X}_{\tau}\right|_{E}}{\delta} \leq \frac{1}{\delta}\left|\int_{s-\delta}^{\tau} \nabla_{x} X_{\tau}^{\sigma, X_{\sigma}^{t, x}}(-A)^{-\alpha} \xi_{\sigma} d \sigma\right|_{E} \leq C, \quad \tau \in[s-\delta, T]
$$

On the other hand, the pair of processes $(\dot{Y}, \dot{Z})$ is solution to the FBSDE

$$
\left\{\begin{aligned}
-d \dot{Y}_{\tau}= & \nabla_{x} \psi\left(\tau, X_{\tau}^{t, x}, Y_{\tau}^{t, x}, Z_{\tau}^{t, x}\right) \dot{X}_{\tau} d \tau+\nabla_{y} \psi\left(\tau, X_{\tau}^{t, x}, Y_{\tau}^{t, x}, Z_{\tau}^{t, x}\right) \dot{Y}_{\tau} d \tau \\
& +\nabla_{z} \psi\left(\tau, X_{\tau}^{t, x}, Y_{\tau}^{t, x}, Z_{\tau}^{t, x}\right) \dot{Z}_{\tau} d \tau-\dot{Z}_{\tau} d W_{\tau}, \quad \tau \in[s-\delta, T] \\
\dot{Y}_{T}= & \nabla_{x} \Phi\left(X_{T}^{t, x}\right) \dot{X}_{T}, \\
d \dot{X}_{\tau}= & A \dot{X}_{\tau} d \tau+\nabla F\left(X_{\tau}^{t, x}\right) \dot{X}_{\tau} d \tau, \quad \tau \in[s-\delta, T] \\
\dot{X}_{s-\delta}= & 0 .
\end{aligned}\right.
$$

Moreover, taking into account (3.25) and the linearity of the BSDE (3.26), we get that the pair $(\dot{Y}, \dot{Z})$ satisfies the estimates

$$
\sup _{\tau \in[s-\delta, T]} \frac{\left|\dot{Y}_{\tau}\right|}{\delta} \leq C, \quad \mathbb{P} \text {-a.s. }
$$




$$
\frac{1}{\delta} \mathbb{E}\left[\int_{s-\delta}^{T}\left|\dot{Z}_{\tau}\right|_{H}^{2} d \tau\right] \leq C
$$

By Hypothesis 3.1,

$$
\left|\nabla_{x} \psi_{\sigma}\right|_{E^{*}} \leq C, \quad\left|\nabla_{y} \psi_{\sigma}\right| \leq C, \quad\left|\nabla_{z} \psi_{\sigma}\right|_{H} \leq C\left(1+|z|_{H}\right), \quad \sigma \in[s-\delta, T] .
$$

Therefore, collecting (3.24)-(3.25), (3.27)-(3.28) and (3.29), (3.23) gives

$$
\begin{aligned}
& \frac{1}{\delta} \mathbb{E}\left[\int_{s-\delta}^{s} \psi\left(\sigma, X_{\sigma}^{t, x}, Y_{\sigma}^{t, x}, Z_{\sigma}^{t, x}\right) d \sigma \int_{s-\delta}^{s} \xi_{\sigma} d W_{\sigma}\right] \\
& =\frac{1}{\delta} \mathbb{E}\left[\int_{s-\delta}^{s} \frac{d}{d \varepsilon} \psi\left(\sigma, X_{\sigma}^{\varepsilon}, v\left(\sigma, X_{\sigma}^{\varepsilon}\right), u\left(\sigma, X_{\sigma}^{\varepsilon}\right)\right) d \sigma\right] \\
& =\mathbb{E}\left[\int_{s-\delta}^{s}\left(\nabla_{x} \psi_{\sigma} \frac{\dot{X}_{\sigma}}{\delta}+\nabla_{y} \psi_{\sigma} \frac{\dot{Y}_{\sigma}}{\delta}+\nabla_{z} \psi_{\sigma} \frac{\dot{Z}_{\sigma}}{\delta}\right) d \sigma\right] \\
& \leq C \delta+C \mathbb{E}\left[\int_{s-\delta}^{s}\left|\nabla_{y} \psi_{\sigma}\right| \frac{\left|\dot{Y}_{\sigma}\right|}{\delta} d \sigma\right]+C \mathbb{E}\left[\int_{s-\delta}^{s}\left|\nabla_{z} \psi_{\sigma}\right|_{H} \frac{\left|\dot{Z}_{\sigma}\right|_{H}}{\delta} d \sigma\right] \leq C \delta+\mathbb{E}\left[\int_{s-\delta}^{s}\left|Z_{\sigma}\right|_{H}^{2} d \sigma\right]
\end{aligned}
$$

which goes to zero as $\delta$ goes to zero. This shows that (3.17) holds true and concludes the proof.

Corollary 3.11. Under the assumptions of Theorem 3.10 we have

$$
Z_{s}^{t, x} h=\nabla v\left(s, X_{s}^{t, x}\right)(-A)^{-\alpha} h, \quad h \in H, \text { for a.e. } s \in[t, T], \mathbb{P} \text {-a.s., }
$$

where $\nabla_{x} v(s, x)(-A)^{-\alpha}$ denotes an extension of the operator $\nabla_{x} v(s, x)(-A)^{-\alpha}: E_{0} \rightarrow \mathbb{R}$ to the whole space $H$. Moreover, there exists a constant $C$, that may depend also on $\nabla_{x} \phi, \nabla_{x} \psi$ and $L_{\psi}$, such that

$$
\left|Z_{s}^{t, x}\right|_{H} \leq C, \quad \text { for a.e. } s \in[t, T], \mathbb{P} \text {-a.s. }
$$

Proof. Since $E_{0}$ is dense in $H$, by (3.12) in Theorem 3.10, for almost every $s \in[0, T]$ and almost surely with respect to the law of $X$, the operator $\nabla_{x} v(s, x)(-A)^{-\alpha}: E_{0} \rightarrow \mathbb{R}$ extends to an operator defined on the whole $H$, which we still denote $\nabla v(s, x)(-A)^{-\alpha}$.

Moreover, from (3.12) and by the Markov property, we get

$$
Z_{\sigma}^{t, x}=Z_{\sigma}^{\sigma, X_{\sigma}^{t, x}}=\left.\nabla_{x} Y_{\sigma}^{\sigma, k}\right|_{k=X_{\sigma}^{t, x}}(-A)^{-\alpha}, \quad \text { for a.e. } \sigma \in[0, T], \mathbb{P} \text {-a.s. }
$$

The conclusion (3.30) follows from the fact that $\sup _{\sigma}\left|\nabla_{x} Y_{\sigma}^{\sigma, k}\right| \leq C$ by (3.10), where $C$ is a constant that does not depend on $k$.

Now we use the previous result to give a priori estimates on $Z^{t, x}$.

Proposition 3.12. Assume that Hypotheses 2.1 and 3.1 hold true, and for any $(t, x) \in[0, T] \times E$, let $\left(X^{t, x}, Y^{t, x}, Z^{t, x}\right)$ be the solution to the FBSDE (3.1). Then there exists a positive constant $C_{T}$ only depending on $T, A, F K_{\phi}, L_{\psi}, K_{\psi}$ such that

$$
\begin{aligned}
\left|Z_{t}^{t, x} h\right| & \leq C_{T}(T-t)^{-1 / 2}|h|_{H}, \quad \mathbb{P} \text {-a.s., } h \in H, \\
\left|\nabla_{x} Y_{t}^{t, x} h\right| & \leq C_{T}(T-t)^{-1 / 2-\alpha}|h|_{H}, \quad \mathbb{P} \text {-a.s., } h \in H .
\end{aligned}
$$


Proof. In the following $C_{T}$ will denote a positive constant which may depend on $T, L_{\psi}, K_{\psi}, K_{\phi}$ but not on $\nabla_{x} \phi$, and that may vary from line to line. We fix $(t, x) \in[0 T] \times E$.

We start by proving estimate (3.31). We first take $\phi$ and $\psi$ differentiable. By Proposition 3.7, the triple of processes $\left(X^{t, x}, Y^{t, x}, Z^{t, x}\right)$ is Gâteaux differentiable as a map from $E$ with values in $\mathscr{S}^{2}((t, T] ; E) \times$ $\mathscr{S}^{2}([t, T]) \times \mathscr{M}^{2}([t, T] ; H)$, and for any $h \in E_{0}$, the triple of processes $\left(\nabla_{x} X^{t, x}, \nabla_{x} Y^{t, x} h, \nabla_{x} Z^{t, x} h\right)$ is solution to (3.9), and satisfies estimate (3.10).

Let us now introduce the process

$$
W_{\tau}^{\mathbb{Q}}:=W_{\tau}-\int_{t}^{\tau} \nabla_{z} \psi\left(s, X_{s}^{t, x}, Y_{s}^{t, x}, Z_{s}^{t, x}\right) d s, \quad \tau \in[t, T],
$$

where $\mathbb{Q}$ is the probability measure such that $W^{\mathbb{Q}}$ is a Brownian motion in $\left(\Omega, \mathscr{F},\left(\mathscr{F}_{t}\right)_{t \geq 0}, \mathbb{Q}\right)$.

Let us fix $h \in E_{0}$. Arguing as in [21, Proposition 3.6] it follows that

$$
\begin{aligned}
F_{\tau}^{t, x} h:= & e^{\int_{t}^{\tau} \nabla_{y} \psi\left(s, X_{s}^{t, x}, Y_{s}^{t, x}, Z_{s}^{t, x}\right) d s} \nabla_{x} Y_{\tau}^{t, x} h \\
& +\int_{t}^{\tau} e^{\int_{t}^{s} \nabla_{y} \psi\left(r, X_{r}^{t, x}, Y_{r}^{t, x}, Z_{r}^{t, x}\right) d r} \nabla_{x} \psi\left(s, X_{s}^{t, x}, Y_{s}^{t, x}, Z_{s}^{t, x}\right) \nabla_{x} X_{s}^{t, x} h d s, \quad \tau \in[t, T] .
\end{aligned}
$$

Therefore, $\left(\left|F_{\tau}^{t, x} h\right|^{2}\right)_{\tau \in[t, T]}$ is a $\mathbb{Q}$-submartingale, which implies, thanks to identification formula (3.12), that

$$
\mathbb{E}^{\mathbb{Q}}\left[\int_{t}^{\tau}\left|F_{s}^{t, x} h\right|^{2} d s\right] \geq(\tau-t)\left|F_{t}^{t, x} h\right|^{2}=(\tau-t)\left|Z_{t}^{t, x}(-A)^{\alpha} h\right|, \quad \tau \in[t, T] .
$$

Further, since $\psi$ is differentiable and Lipschitz continuous with respect to $x$ and $y$, and $\nabla_{x} X^{t, x}$ is bounded (see (2.6)), we deduce that

$$
\left|F_{\tau}^{t, x} h\right|^{2} \leq C_{T}\left(\left|\nabla_{x} Y_{\tau}^{t, x} h\right|^{2}+|h|_{H}^{2}\right), \quad \tau \in[t, T], \mathbb{P} \text {-a.s. }
$$

It remains to estimate $\left|\nabla_{x} Y_{\tau}^{t, x} h\right|$. To this aim, we recall the well-known estimate

$$
\mathbb{E}^{\mathbb{Q}}\left[\left(\int_{t}^{\tau}\left|Z_{s}^{t, x}\right|_{H}^{2} d s\right)^{p / 2}\right] \leq C\|\Phi\|_{\infty}^{p}, \quad \tau \in[t, T],
$$

for some $C>0$ and any $p<+\infty$. Formulas (3.35), (3.12) and (2.15) give

$$
E^{\mathbb{Q}}\left[\int_{t}^{\tau}\left|\nabla Y_{s}^{t, x} h\right|^{2} d s\right] \leq \mathbb{E}^{\mathbb{Q}}\left[\int_{t}^{\tau}\left|Z_{s}^{t, x}\right|_{H}^{2}\left|(-A)^{\alpha} \nabla X_{s}^{t, x} h\right|_{H}^{2} d s\right] \leq c\|\Phi\|_{\infty}^{2}\left|(-A)^{\alpha} h\right|_{H}^{2}, \quad \tau \in[t, T],
$$

which, together with (3.33) and (3.34), allows us to conclude that

$$
\left|Z_{t}^{t, x}(-A)^{\alpha} h\right|^{2} \leq \frac{C_{T}}{T-t}\left|(-A)^{\alpha} h\right|_{H}^{2}, \quad h \in E_{0} .
$$

Let now fix $h \in H$. We notice that in this case we can write $h=(-A)^{\alpha}(-A)^{-\alpha} h$. Therefore,

$$
\left|Z_{t}^{t, x} h\right|^{2}=\left|Z_{t}^{t, x}(-A)^{\alpha}(-A)^{-\alpha} h\right|^{2} \leq \frac{C_{T}}{T-t}\left|(-A)^{\alpha}(-A)^{-\alpha} h\right|_{H}^{2} \leq \frac{C_{T}}{T-t}|h|_{H}^{2}, \quad h \in H,
$$

which provides (3.31) in the case of $\psi$ and $\phi$ differentiable.

Finally, the case $\psi$ and $\phi$ non differentiable can be obtained by approximating $\psi$ and $\phi$ with $\psi_{n}$ and $\phi_{n}$ in (3.7) and (3.6), respectively. For the proof we refer to [21, Proposition 3.6]. 
Let us now prove estimate (3.32). Again, at first we prove the result when $\psi$ and $\phi$ are differentiable and then we generalize it by approximation. Let us fix $h \in E_{0}$. For any $t<\eta<\tau \leq T$, the submartingale property of $\left(\left|F_{s}^{t, x}\right|_{H}^{2}\right)_{s \in[t, T]}$ gives

$$
\mathbb{E}^{\mathbb{Q}}\left[\int_{\eta}^{\tau}\left|F_{s}^{t, x} h\right|^{2} d s\right]=\int_{\eta}^{\tau} \mathbb{E}^{\mathbb{Q}}\left[\left|F_{s}^{t, x} h\right|^{2}\right] d s \geq \int_{\eta}^{\tau} \mathbb{E}^{\mathbb{Q}}\left[\left|F_{\eta}^{t, x} h\right|^{2}\right] d s=(\tau-\eta) \mathbb{E}^{\mathbb{Q}}\left[\left|F_{\eta}^{t, x} h\right|^{2}\right] .
$$

Moreover, for any $\tau \in(t, T]$ we split

$$
\mathbb{E}^{\mathbb{Q}}\left[\int_{t}^{\tau}\left|F_{s}^{t, x} h\right|^{2} d s\right]=\mathbb{E}^{\mathbb{Q}}\left[\int_{t}^{(t+\tau) / 2}\left|F_{s}^{t, x} h\right|^{2} d s\right]+\mathbb{E}^{\mathbb{Q}}\left[\int_{(t+\tau) / 2}^{\tau}\left|F_{s}^{t, x} h\right|^{2} d s\right]=: I_{1}+I_{2} .
$$

Let us evaluate separately $I_{1}$ and $I_{2}$. Concerning $I_{1}$, identification formula (3.12), (3.35) and (2.14) give

$$
\begin{aligned}
& \mathbb{E}^{\mathbb{Q}}\left[\int_{\eta}^{\tau}\left|\nabla Y_{s}^{t, x} h\right|^{2} d s\right] \\
& \leq \mathbb{E}^{\mathbb{Q}}\left[\int_{\eta}^{\tau}\left|Z_{s}^{t, x}\right|_{H}^{2}\left|(-A)^{\alpha} \nabla X_{s}^{t, x} h\right|_{H}^{2} d s\right] \\
& +C_{T}(\tau-t)^{-2 \alpha}\left(|x|_{E}^{2 m+1}+\mathbb{E}^{\mathbb{Q}}\left[\sup _{\tau \in[t, T]}\left|w^{A}(\tau)\right|_{E}^{2 m+1}\right]\right)^{2}|h|_{H}^{2} \mathbb{E}^{\mathbb{Q}}\left[\int_{\eta}^{\tau}\left|Z_{s}^{t, x}\right|_{H}^{2} d s\right] \\
& \leq C_{T}\|\Phi\|_{\infty}^{2}|h|_{H}^{2}(\eta-t)^{-2 \alpha} .
\end{aligned}
$$

Hence, from (3.34), (3.36) and (3.38) it follows that

$$
E^{\mathbb{Q}}\left[\left|F_{\eta}^{t, x} h\right|_{H}^{2}\right] \leq C_{T}\|\Phi\|_{\infty}^{2}|h|_{H}^{2}\left(\frac{(\eta-t)^{-2 \alpha}}{(\tau-\eta)}+1\right), \quad t<\eta<\tau \leq T .
$$

By applying Fubini's theorem and (3.39), we infer that

$$
\begin{aligned}
I_{1} & \leq C_{T}\|\Phi\|_{\infty}^{2}|h|_{H}^{2}\left[(\tau-t)^{-1} \int_{t}^{(t+\tau) / 2}(s-t)^{-2 \alpha} d s+(\tau-t)^{-1} \int_{t}^{(t+\tau) / 2} d s\right] \\
& =C_{T}|h|_{H}^{2}\left[(\tau-t)^{-2 \alpha}+(\tau-t)\right] \leq C_{T}|h|_{H}^{2}(\tau-t)^{-2 \alpha}, \quad t<\eta<\tau \leq T .
\end{aligned}
$$

As far as $I_{2}$ is concerned, we take advantage from (3.34) and (3.38). Then, for $t<\eta<\tau \leq T$ we get

$$
I_{2} \leq C_{T}|h|_{H}^{2}\left[(\tau-t)+(\tau-t)^{-2 \alpha}\right]=C_{T}|h|_{H}^{2}\left[(\tau-t)^{-2 \alpha}+(\tau-t)\right] \leq C_{T}|h|_{H}^{2}(\tau-t)^{-2 \alpha} .
$$

Thus collecting (3.36), (3.40) and (3.41), we have

$$
(\tau-\eta) \mathbb{E}^{\mathbb{Q}}\left[\left|F_{\eta}^{t, x} h\right|^{2}\right] \leq \mathbb{E}^{\mathbb{Q}} \int_{t}^{\tau}\left|F_{s}^{t, x} h\right|^{2} d s \leq C_{T}|h|_{H}^{2}(\tau-t)^{-2 \alpha}, \quad t<\eta<\tau \leq T,
$$

so that

$$
\mathbb{E}^{\mathbb{Q}}\left[\left|F_{\eta}^{t, x} h\right|^{2}\right] \leq C_{T} \frac{1}{(\tau-\eta)(\tau-t)^{2 \alpha}}|h|_{H}^{2}, \quad h \in E_{0}, \quad t \leq \eta<\tau \leq T .
$$

Let us now fix $h \in H$, and let us consider a sequence $\left(h_{n}\right) \subset E_{0}$ such that $h_{n} \rightarrow h$ as $n \rightarrow+\infty$ in $H$. Taking (3.42) with $h$ replaced by $h_{n}$ and letting $n \rightarrow+\infty$, it follows that

$$
\mathbb{E}^{\mathbb{Q}}\left[\left|F_{\eta}^{t, x} h\right|^{2}\right] \leq \frac{C_{T}}{(\tau-\eta)(\tau-t)^{2 \alpha}}|h|_{H}^{2}, \quad h \in H, \quad t \leq \eta<\tau \leq T .
$$

Inequality (3.32) follows from (3.43) by taking $\tau=T$ and $\eta=t$. 


\section{The Bismut-Elworthy formula and the semilinear Kolmogorov equation: the Lipschitz case}

Recall that we deal with a process $X$ taking values in a Banach space and solution to equation (2.1), with special diffusion operator $(-A)^{-\alpha}$ with pseudo-inverse $(-A)^{\alpha}$ which is not bounded.

In the present section we adequate to our framework the results in [10]. More precisely, in Subsection 4.1 we present a nonlinear version of the Bismut-Elworthy formula in the case of Lipschitz generator, which extends the one provided in [10] in the case of a process $X$ taking values in a Hilbert space, and with a bounded diffusion operator with bounded inverse. Providing the Bismut-Elworthy formula in the case of Lipschitz generator is a fundamental step in order to obtain the analogous formula in the quadratic case. Moreover, it allows us to give an existence and uniqueness result in the Banach framework for the semilinear Kolmogorov related to the process $X$, and with coefficients $\phi$ and $\psi$ not necessarily differentiable, see Subsection 4.2.

For $0 \leq t<s \leq T$ and $h \in H$ we define the real valued random variables

$$
U_{s}^{h, t, x}:=\frac{1}{s-t} \int_{t}^{s}\left\langle(-A)^{\alpha} \nabla_{x} X_{r}^{t, x} h, d W_{r}\right\rangle
$$

Notice that, for any $h \in H$, the process $U^{h, t, x}$ is well defined thanks to formula (2.11) in Proposition 2.6. In what follows we prove some useful estimates on the process $U^{h, t, x}$.

Lemma 4.1. Assume that Hypotheses 2.1 hold true. For any $(t, x) \in[0, T] \times E$, let $X^{t, x}$ be the unique mild solution to (2.1). Then, for any $h \in H$ and for any $q \geq 1$,

$$
\left(\mathbb{E}\left[\left|U_{s}^{h, t, x}\right|^{q}\right]\right)^{1 / q} \leq C(s-t)^{-\left(\frac{1}{2}+\alpha\right)}|h|_{H},
$$

and also

$$
\left(\mathbb{E}\left[\sup _{s \in\left[\frac{t+T}{2}, T\right]}\left|U_{s}^{h, t, x}\right|^{q}\right]\right)^{1 / q} \leq C(T-t)^{-\left(\frac{1}{2}+\alpha\right)}|h|_{H}
$$

Proof. We compute

$$
\begin{aligned}
\mathbb{E}\left[\left|U_{s}^{h, t, x}\right|^{q}\right] & =\mathbb{E}\left[\left|\frac{1}{s-t} \int_{t}^{s}\left\langle(-A)^{\alpha} \nabla_{x} X_{r}^{t, x} h, d W_{r}\right\rangle\right|^{q}\right] \leq \frac{1}{(s-t)^{q}} \mathbb{E}\left[\left(\int_{t}^{s}\left|(-A)^{\alpha} \nabla_{x} X_{r}^{t, x} h\right|^{2} d r\right)^{q / 2}\right] \\
& \leq \frac{1}{(s-t)^{q}} C\left((s-t)^{1-2 \alpha}|h|_{H}^{2}\right)^{q / 2}=C(s-t)^{-q\left(\frac{1}{2}+\alpha\right)}|h|_{H}^{q},
\end{aligned}
$$

where in the latter inequality we have used formula (2.11) of Proposition 2.6 with $\varepsilon=\alpha$. Analogously, we have

$$
\mathbb{E}\left[\sup _{s \in\left[\frac{t+T}{2}, T\right]}\left|U_{s}^{h, t, x}\right|^{q}\right] \leq C \frac{1}{(T-t)^{q}} \mathbb{E}\left[\left(\int_{t}^{T}\left|(-A)^{\alpha} \nabla_{x} X_{r}^{t, x} h\right|^{2} d r\right)^{q / 2}\right] \leq C(T-t)^{-q\left(\frac{1}{2}+\alpha\right)}|h|_{H}^{q} .
$$

\subsection{The Bismut formula}

We can now give a version of the Bismut-Elworthy formula in the case of Lipschitz generator and in the Banach space framework. We consider only the case of final datum $\phi$ and generator $\psi$ bounded with respect to $x$, since we aim to treat such a model in the quadratic case. We start with the case when the coefficients are also differentiable. An analogous result is proved in [10] in the Hilbert space framework 
using the Malliavin calculus. Since here the process $X$ takes its values in a Banach space, we avoid the use of the Malliavin calculus, by exploiting instead techniques similar to the ones used in the proof of Theorem 3.10.

In the rest of the section we will assume the following, that substitutes Hypothesis 3.1.

Hypothesis 4.2. The functions $\phi: E \rightarrow \mathbb{R}$ and $\psi:[0, T] \times E \times \mathbb{R} \times H \rightarrow \mathbb{R}$ in (3.1) satisfy the following.

(i) $\phi$ is continuous, and there exist a nonnegative constant $K_{\phi}$ such that $|\phi(x)| \leq K_{\phi}$ for every $x \in E$.

(ii) $\psi$ is measurable and, for every fixed $t \in[0, T]$, the map $\psi(t, \cdot, \cdot, \cdot): E \times \mathbb{R} \times H \rightarrow \mathbb{R}$ is continuous. Moreover, there exist nonnegative constants $L_{\psi}$ and $K_{\psi}$ such that

$$
\begin{aligned}
& \left|\psi\left(t, x_{1}, y_{1}, z_{1}\right)-\psi\left(t, x_{2}, y_{2}, z_{2}\right)\right| \leq L_{\psi}\left(\left|x_{1}-x_{2}\right|_{E}+\left|y_{1}-y_{2}\right|+\left|z_{1}-z_{2}\right|_{H}\right), \\
& |\psi(t, x, 0,0)| \leq K_{\psi},
\end{aligned}
$$

for every $t \in[0, T], x_{1}, x_{2} \in E, y_{1}, y_{2} \in \mathbb{R}$ and $z_{1}, z_{2} \in H$.

Theorem 4.3. Let Hypotheses 2.1 and 4.2 hold true, and for any $(t, x) \in[0, T] \times E$, let $\left(X^{t, x}, Y^{t, x}, Z^{t, x}\right)$ be a solution of the forward-backward system (3.1), and let $U^{h, t, x}$ be the process defined in (4.1). Assume moreover that $\phi$ is Gâteaux differentiable with bounded derivative, and that $\psi$ is Gâteaux differentiable with respect to $x, y$ and $z$. Then for $t \leq s \leq T, x \in E, h \in H$,

$$
\mathbb{E}\left[\nabla_{x} Y_{s}^{t, x} h\right]=\mathbb{E}\left[\int_{s}^{T} \psi\left(r, X_{r}^{t, x}, Y_{r}^{t, x}, Z_{r}^{t, x}\right) U_{r}^{h, t, x} d r\right]+\mathbb{E}\left[\phi\left(X_{T}^{t, x}\right) U_{T}^{h, t, x}\right] .
$$

Proof. Let $\xi$ be a given square integrable $E_{0}$-valued predictable process, and $X^{\varepsilon, t, x}$ be a mild solution to the equation

$$
\left\{\begin{array}{l}
d X_{\tau}^{\varepsilon, t, x}=A X_{\tau}^{\varepsilon, t, x} d \tau+F\left(X_{\tau}^{\varepsilon, t, x}\right) d \tau+(-A)^{-\alpha} \varepsilon \xi_{\tau} d \tau+(-A)^{-\alpha} d W_{\tau}, \quad \tau \in[t, T], \\
X_{t}^{\varepsilon, t, x}=x
\end{array}\right.
$$

We also consider the pair of processes $\left(Y^{\varepsilon, t, x}, Z^{\varepsilon, t, x}\right)$ solution to the Markovian BSDE

$$
\left\{\begin{array}{l}
-d Y_{\tau}^{\varepsilon, t, x}=\psi\left(\tau, X_{\tau}^{\varepsilon, t, x}, Y_{\tau}^{\varepsilon, t, x}, Z_{\tau}^{\varepsilon, t, x}\right) d \tau-Z_{\tau}^{\varepsilon, t, x} d W_{\tau}, \quad \tau \in[t, T], \\
Y_{T}^{\varepsilon, t, x}=\phi\left(X_{T}^{\varepsilon, t, x}\right) .
\end{array}\right.
$$

Arguing similarly to the proof of Theorem 3.10, we define

$$
\dot{X}_{\tau}:=\frac{d}{d \varepsilon}{ }_{\mid \varepsilon=0}^{\varepsilon, t, x}, \dot{Y}_{\tau}:=\frac{d}{d \varepsilon}{ }_{\mid \varepsilon=0} Y_{\tau}^{\varepsilon, t, x}, \dot{Z}_{\tau}:=\frac{d}{d \varepsilon}{ }_{\mid \varepsilon=0} Z_{\tau}^{\varepsilon, t, x}, \quad \tau \in[t, T],
$$

which are solution to the forward-backward system (3.26) with $s-\delta=t$. We already know (see formula (3.21) with $s-\delta=t$ ) that

$$
\dot{X}_{\tau}=\int_{t}^{\tau} \nabla_{x} X_{\tau}^{\sigma, X_{\sigma}^{t, x}}(-A)^{-\alpha} \xi_{\sigma} d \sigma, \quad \tau \in[t, T], \mathbb{P} \text {-a.s. }
$$

Now we want to prove a similar identification for the pair $(Y, Z)$. To this aim, for any $\sigma \in[t, T]$, we consider the Markovian BSDE in (3.1) on the time interval $[\sigma, T]$, and with initial condition $y$ given at time $\sigma$; from Proposition 3.7 we know that the derivative with respect to $y \in E$ in the direction $h \in E$ satisfies the following BSDE, that we write in integral form: for any $\tau \in[t, T], \mathbb{P}$-a.s.,

$$
\left\{\begin{aligned}
\nabla_{x} Y_{\tau}^{\sigma, y} h= & \nabla_{x} \phi\left(X_{T}^{\sigma, y}\right) \nabla_{x} X_{T}^{\sigma, y} h-\int_{\tau}^{T} \nabla_{x} Z_{r}^{\sigma, x} h d W_{r}+\int_{\tau}^{T}\left(\nabla_{x} \psi\left(r, X_{r}^{\sigma, y}, Y_{r}^{\sigma, y}, Z_{r}^{\sigma, y}\right) \nabla_{x} X_{r}^{\sigma, y} h\right. \\
& \left.+\nabla_{y} \psi\left(r, X_{r}^{\sigma, y}, Y_{r}^{\sigma, y}, Z_{r}^{\sigma, y}\right) \nabla_{x} Y_{r}^{\sigma, y} h d r+\nabla_{z} \psi\left(r, X_{r}^{\sigma, y}, Y_{r}^{\sigma, y}, Z_{r}^{\sigma, y}\right) \nabla_{x} Z_{r}^{\sigma, y} h\right) d r .
\end{aligned}\right.
$$


Let us take $y=X_{\sigma}^{t, x}$ and $h=(-A)^{-\alpha} \xi_{\sigma}$ in (4.9), and let us integrate both sides with respect to $\sigma \in[t, \tau]$. By inverting the order of integration where necessary, and using the Markov property, it is immediate to get

$$
\begin{aligned}
& \int_{t}^{\tau} \nabla_{x} Y_{\tau}^{\sigma, X_{\sigma}^{t, x}}(-A)^{-\alpha} \xi_{\sigma} d \sigma \\
& =\int_{t}^{\tau} \nabla_{x} \phi\left(X_{T}^{\sigma, X_{\sigma}^{t, x}}\right) \nabla_{x} X_{T}^{\sigma, X_{\sigma}^{t, x}}(-A)^{-\alpha} \xi_{\sigma} d \sigma-\int_{\tau}^{T}\left(\int_{t}^{\tau} \nabla_{x} Z_{r}^{\sigma, X_{\sigma}^{t, x}}(-A)^{-\alpha} \xi_{\sigma} d \sigma\right) d W_{r} \\
& +\int_{\tau}^{T}\left(\int_{t}^{\tau} \nabla_{x} \psi\left(r, X_{r}^{\sigma, X_{\sigma}^{t, x}}, Y_{r}^{\sigma, X_{\sigma}^{t, x}}, Z_{r}^{\sigma, X_{\sigma}^{t, x}}\right) \nabla_{x} X_{r}^{\sigma, X_{\sigma}^{t, x}}(-A)^{-\alpha} \xi_{\sigma} d \sigma\right) d r \\
& +\int_{t}^{\tau}\left(\int_{t}^{\tau} \nabla_{y} \psi\left(r, X_{r}^{\sigma, X_{\sigma}^{t, x}}, Y_{r}^{\sigma, X_{\sigma}^{t, x}}, Z_{r}^{\sigma, X_{\sigma}^{t, x}}\right) \nabla_{x} Y_{r}^{\sigma, X_{\sigma}^{t, x}}(-A)^{-\alpha} \xi_{\sigma} d \sigma\right) d r \\
& +\int_{t}^{\tau}\left(\int_{t}^{\tau} \nabla_{z} \psi\left(r, X_{r}^{\sigma, X_{\sigma}^{t, x}}, Y_{r}^{\sigma, X_{\sigma}^{t, x}}, Z_{r}^{\sigma, X_{\sigma}^{t, x}}\right) \nabla_{x} Z_{r}^{\sigma, X_{\sigma}^{t, x}}(-A)^{-\alpha} \xi_{\sigma} d \sigma\right) d r .
\end{aligned}
$$

By (4.6) and (4.7), together with (4.8), we can conclude that

$$
\dot{Y}_{\tau}=\int_{t}^{\tau} \nabla_{x} Y_{\tau}^{\sigma, X_{\sigma}^{t, x}}(-A)^{-\alpha} \xi_{\sigma} d \sigma, \quad \dot{Z}_{\tau}=\int_{t}^{\tau} \nabla_{x} Z_{\tau}^{\sigma, X_{\sigma}^{t, x}}(-A)^{-\alpha} \xi_{\sigma} d \sigma, \quad \tau \in(t, T], \mathbb{P} \text {-a.s. }
$$

since these two pairs of processes satisfies the same BSDE. By density, arguing as in Corollary 3.11, we infer that formulas (4.8) and (4.10) hold true for any square integrable $H$-valued predictable process $\xi$. Now, let $\eta \in E$, and let us take

$$
\xi_{\tau}:=(-A)^{\alpha} \nabla_{x} X_{\tau}^{t, x} \eta, \quad \tau \in(t, T]
$$

Notice that, since $(-A)^{\alpha} \nabla_{x} X_{\tau}^{t, x} \eta \in D\left((-A)^{1 / 2-\alpha}\right) \mathbb{P}$-a.s., thanks to Proposition $2.6,(-A)^{\alpha} \nabla_{x} X_{\tau}^{t, x} \eta \in H$ for any $\tau \in(t, T], \mathbb{P}$-a.s., and so

$$
\int_{t}^{\tau} e^{(\tau-\sigma) A}(-A)^{-\alpha}(-A)^{\alpha} \nabla_{x} X_{\sigma}^{t, x} \eta d \sigma=\int_{t}^{\tau} e^{(\tau-\sigma) A} \nabla_{x} X_{\sigma}^{t, x} \eta d \sigma, \quad \tau \in(t, T], \mathbb{P} \text {-a.s. },
$$

which belongs to $E$. Therefore, for all $\tau \in(t, T]$ we have $\dot{X}_{\tau} \in E \mathbb{P}$-a.s., where $\dot{X}$ denotes the mild solution to the forward equation in (3.26) with $s-\delta=t$ with $\xi$ given by (4.11). With this choice of $\xi$ equalities (4.8) and (4.10) can be rewritten as

$$
\begin{aligned}
& \dot{X}_{\tau}=\int_{t}^{\tau} \nabla_{x} X_{\tau}^{\sigma, X_{\sigma}^{t, x}} \nabla_{x} X_{\sigma}^{t, x} \eta d \sigma=(\tau-t) \nabla_{x} X_{\tau}^{t, x} \eta, \quad \dot{Y}_{\tau}=\int_{t}^{\tau} \nabla_{x} Y_{\tau}^{\sigma, X_{\sigma}^{t, x}} \nabla_{x} X_{\sigma}^{t, x} \eta d \sigma=(\tau-t) \nabla_{x} Y_{\tau}^{t, x} \eta \\
& \dot{Z}_{\tau}=\int_{t}^{\tau} \nabla_{x} Z_{\tau}^{\sigma, X_{\sigma}^{t, x}} \nabla_{x} X_{\sigma}^{t, x} \eta d \sigma=(\tau-t) \nabla_{x} Z_{\tau}^{t, x} \eta, \quad \tau \in[t, T], \mathbb{P} \text {-a.s. }
\end{aligned}
$$

Let us now set

$$
\begin{aligned}
\dot{\psi}(\tau, t, x):= & \nabla_{x} \psi\left(\tau, X_{\tau}^{t, x}, Y_{\tau}^{t, x}, Z_{\tau}^{t, x}\right) X_{\tau}^{t, x} d \tau+\nabla_{y} \psi\left(\tau, X_{\tau}^{t, x}, Y_{\tau}^{t, x}, Z_{\tau}^{t, x}\right) Y_{\tau}^{t, x} d \tau \\
& +\nabla_{z} \psi\left(\tau, X_{\tau}^{t, x}, Y_{\tau}^{t, x}, Z_{\tau}^{t, x}\right) Z_{\tau}^{\dot{t}, x} d \tau, \\
\dot{\phi}\left(X_{T}^{t, x}\right):= & \nabla_{x} \phi\left(X_{T}^{t, x}\right) X_{T}^{t, x} .
\end{aligned}
$$


By (4.12), $\dot{\psi}$ and $\dot{\phi}$ can be rewritten as

$$
\begin{aligned}
\dot{\psi}(\tau, t, x) & =(\tau-t)\left(\nabla_{x} \psi\left(\tau, X_{\tau}^{t, x}, Y_{\tau}^{t, x}, Z_{\tau}^{t, x}\right) \nabla_{x} X_{\tau}^{t, x} \eta d \tau+\nabla_{y} \psi\left(\tau, X_{\tau}^{t, x}, Y_{\tau}^{t, x}, Z_{\tau}^{t, x}\right) \nabla_{x} Y_{\tau}^{t, x} \eta d \tau\right. \\
& \left.+\nabla_{z} \psi\left(\tau, X_{\tau}^{t, x}, Y_{\tau}^{t, x}, Z_{\tau}^{t, x}\right) \nabla_{x} Z_{\tau}^{t, x} \eta d \tau\right) \\
\dot{\phi}\left(X_{T}^{t, x}\right) & =(T-t) \nabla_{x} \phi\left(X_{T}^{t, x}\right) \nabla_{x} X_{T}^{t, x} \eta
\end{aligned}
$$

Notice that the right-hand sides in (4.13) and in (4.14) are nothing else (modulo a renormalization) than the terms appearing in the right-hand sides of the first two equations in (3.9). Now we aim at finding an

expression for $\psi$ and $\phi$ that does not involve the derivative of $\psi, \phi, X, Y$ and $Z$ : this in turn will furnish an expression of $\nabla_{x} Y$ that does not involve the derivatives of $\psi, \phi, X, Y$ and $Z$, as in formula (4.4). To this end, let us consider the process

$$
W_{\sigma}^{\varepsilon}=W_{\sigma}-\varepsilon \int_{t}^{\sigma}(-A)^{\alpha} \nabla_{x} X_{r}^{t, x} \eta d r, \quad 0 \leq t \leq \sigma \leq T,
$$

and let us define a probability measure $Q_{\varepsilon}$ such that

$$
\frac{d Q_{\varepsilon}}{d \mathbb{P}}=\exp \left(\varepsilon \int_{t}^{T}\left\langle(-A)^{\alpha} \nabla_{x} X_{\sigma}^{t, x} \eta, d W_{\sigma}\right\rangle-\frac{\varepsilon^{2}}{2} \int_{t}^{T}\left|(-A)^{\alpha} \nabla_{x} X_{\sigma}^{t, x} \eta\right|_{H}^{2} d \sigma\right) .
$$

By the Girsanov theorem, under $Q_{\varepsilon}\left(W_{\sigma}^{\varepsilon}\right)_{\sigma \in[t, T]}$ is a cylindrical Wiener process in $H$. Arguing as in the proof of Theorem 3.10, we also notice that the process $X$ under $Q_{\varepsilon}$ and the process $X^{\varepsilon}$ under $\mathbb{P}$ have the same law. Therefore,

$$
\begin{aligned}
\mathbb{E}\left[\nabla_{x} Y_{\tau}^{t, x} \eta\right] & =\mathbb{E}\left[\nabla_{x} \Phi\left(X_{T}^{t, x}\right) \nabla_{x} X_{T}^{t, x} \eta\right]+\mathbb{E}\left[\int _ { \tau } ^ { T } \left(\nabla_{x} \psi\left(\sigma, X_{\sigma}^{t, x}, Y_{\sigma}^{t, x}, Z_{\sigma}^{t, x}\right) \nabla_{x} X_{\sigma}^{t, x} \eta\right.\right. \\
& \left.\left.+\nabla_{y} \psi\left(\sigma, X_{\sigma}^{t, x}, Y_{\sigma}^{t, x}, Z_{\sigma}^{t, x}\right) \nabla_{x} Y_{\sigma}^{t, x} \eta+\nabla_{z} \psi\left(\sigma, X_{\sigma}^{t, x}, Y_{\sigma}^{t, x}, Z_{\sigma}^{t, x}\right) \nabla_{x} Z_{\sigma}^{t, x} \eta\right) d \sigma\right] \\
& =\frac{1}{T-t} \mathbb{E}\left[\dot{\phi}\left(X_{T}^{t, x}\right)\right]+\mathbb{E}\left[\int_{\tau}^{T} \frac{1}{\sigma-t} \dot{\psi}(\sigma, t, x) d \sigma\right]
\end{aligned}
$$

By differentiating inside the expectation with respect to $\varepsilon$ and changing the order of integration, we get

$$
\begin{aligned}
\mathbb{E}[\dot{\psi}(\sigma, t, x)] & =\mathbb{E}\left[\frac{d}{d \varepsilon}{ }_{\varepsilon=0} \psi\left(\sigma, X_{\sigma}^{\varepsilon, t, x}, Y_{\sigma}^{\varepsilon, t, x}, Z_{\sigma}^{\varepsilon, t, x}\right)\right]=\frac{d}{d \varepsilon} \mid \varepsilon=0 \\
& =\mathbb{E}\left[\psi\left(\sigma, X_{\sigma}^{t, x}, Y_{\sigma}^{t, x}, Z_{\sigma}^{t, x}\right) \int_{t}^{\sigma}\left\langle(-A)^{\alpha} \nabla_{x} X_{r}^{t, x} \eta, d W_{r}\right\rangle\right]
\end{aligned}
$$

and so, recalling (4.1),

$$
\begin{aligned}
\mathbb{E}\left[\int_{\tau}^{T} \frac{1}{\sigma-t} \dot{\psi}(\sigma, t, x) d \sigma\right] & =\mathbb{E}\left[\int_{\tau}^{T} \frac{1}{\sigma-t}\left(\int_{t}^{\sigma}\left\langle(-A)^{\alpha} \nabla_{x} X_{r}^{t, x} \eta, d W_{r}\right\rangle\right) \psi\left(\sigma, X_{\sigma}^{t, x}, Y_{\sigma}^{t, x}, Z_{\sigma}^{t, x}\right) d \sigma\right] \\
& =\mathbb{E}\left[\int_{\tau}^{T} U_{\sigma}^{\eta, t, x} \psi\left(\sigma, X_{\sigma}^{t, x}, Y_{\sigma}^{t, x}, Z_{\sigma}^{t, x}\right) d \sigma\right]
\end{aligned}
$$

Similarly, $\mathbb{E}\left[\dot{\phi}\left(X_{T}^{t, x}\right)\right]=\mathbb{E}\left[\phi\left(X_{T}^{t, x}\right) U_{T}^{\eta, t, x}\right]$, and this proves (4.4) when $\eta \in E$. The general case with $\eta \in H$ follows by density, thanks to estimates (3.32) and (4.2).

In the next result we remove the differentiability assumption on $\psi$ and $\phi$ in Theorem 4.3. 
Theorem 4.4. Let Hypotheses 2.1 and 4.2 hold true, and for any $(t, x) \in[0, T] \times E$, let $\left(X^{t, x}, Y^{t, x}, Z^{t, x}\right)$ be a solution of the forward-backward system (3.1), and let $U^{h, t, x}$ be the process defined in (4.1). Then, for $t \leq s \leq T, x \in E, h \in H$, the Bismut formula given in (4.4) holds true.

Proof. The proof follows the same lines of the one of Theorem 3.10 in [10]. The main ingredients are formula (3.12) in Theorem 3.10 and Proposition 3.6, which provide respectively the identification of $Z$ in the Banach space case and with the diffusion operator $(-A)^{-\alpha}$, and the stability result for the BSDE in (3.1) when the generator and the final datum are approximated by (3.7)-(3.6). We underline that approximations (3.6)-(3.7) preserve the boundedness and the growth, and are only of pointwise type. Notice that in [10], the final datum and the generator are approximated by means of their inf-sup convolutions, and so the approximation is uniform. However, thanks to the aforementioned stability properties for the BSDE, our pointwise approximations (3.7)-(3.6) are sufficient to obtain the desired result.

\subsection{The semilinear Kolmogorov equation}

By means of Theorem 4.4, we can give an existence and uniqueness result in the Banach framework for the semilinear Kolmogorov related to the the process $X$, and with coefficients $\phi$ and $\psi$ not necessarily differentiable, as it is assumed in [19].

Let $P_{t, \tau}, t \leq \tau \leq T$, be the transition semigroup related to the process $X^{t, x}$ solution of the forward equation (2.1), namely, for every bounded and measurable function $\varphi: E \rightarrow \mathbb{R}, P_{t, \tau}[\varphi](x):=\mathbb{E} \varphi\left(X_{\tau}^{t, x}\right)$. We consider the following semilinear Kolmogorov equation

$$
\left\{\begin{array}{l}
\frac{\partial v}{\partial t}(t, x)=-\mathscr{L} v(t, x)+\psi\left(t, x, v(t, x), \nabla^{(-A)^{-\alpha}} v(t, x)\right), \quad t \in[0, T], x \in E, \\
v(T, x)=\phi(x),
\end{array}\right.
$$

where $\mathscr{L}$ is the generator of the transition semigroup $\left(P_{t, s}\right)_{0 \leq t \leq s \leq T}$, that is, at least formally,

$$
(\mathscr{L} f)(x)=\frac{1}{2}\left(\operatorname{Tr}\left((-A)^{-\alpha}\left(-A^{*}\right)^{-\alpha} \nabla^{2} f\right)(x)+\langle A x, \nabla f(x)\rangle+\langle F(x), \nabla f(x)\rangle, \quad x \in E .\right.
$$

We introduce the notion of mild solution of the nonlinear Kolmogorov equation (4.16), see e.g. [11].

Definition 4.5. A function $v:[0, T] \times E \rightarrow \mathbb{R}$ is a mild solution of the semilinear Kolmogorov equation (4.16) if $v \in \mathscr{G}^{0,1}([0, T] \times E)$, and

$$
v(t, x)=P_{t, T}[\phi](x)+\int_{t}^{T} P_{t, s}\left[\psi\left(s, \cdot, v(s, \cdot), \nabla^{(-A)^{-\alpha}} v(s, \cdot)\right)\right](x) d s, \quad t \in[0, T], x \in E .
$$

Theorem 4.6. Let Hypotheses 2.1 and 4.2 hold true. Then the semilinear Kolmogorov equation (4.16) has a unique mild solution $v$ given by the formula

$$
v(t, x)=Y_{t}^{t, x}, \quad(t, x) \in[0, T] \times E,
$$

where, for any $(t, x) \in[0, T] \times E,\left(X^{t, x}, Y^{t, x}, Z^{t, x}\right)$ denotes the solution to the FBSDE (3.1). In addition, we have, $\mathbb{P}$-a.s.,

$$
Y_{s}^{t, x}=v\left(s, X_{s}^{t, x}\right), \quad s \in[t, T], \quad Z_{s}^{t, x}=\nabla_{x} v\left(s, X_{s}^{t, x}\right) \nabla_{x} X_{s}^{t, x}(-A)^{-\alpha}, \quad \text { a.e. } s \in[t, T] .
$$

Proof. If the data $\phi$ and $\psi$ are also differentiable, the result can be proved as in [19], Theorem 6.2. When the data are not differentiable, the Bismut formula (4.4) is still true, see Theorem 4.4, and the result can be proved arguing as in [10], Theorem 4.2 . 


\section{The Bismut-Elworthy formula and the semilinear Kolmogorov equation: the quadratic case}

We are ready to state and prove the main result of the paper, which is a nonlinear Bismut-Elworthy formula as the one in Theorem 4.4, but in the case of quadratic generator. This in particular will give an existence and uniqueness result for the Kolmogorov equation (4.16) in the quadratic case and in the Banach framework, see Theorem 5.4.

Theorem 5.1. Let Hypotheses 2.1 and 3.1 hold true. For any $(t, x) \in[0, T] \times E$, let $\left(X^{t, x}, Y^{t, x}, Z^{t, x}\right)$ be the solution of the forward-backward system (3.1) and let $U^{h, t, x}$ be the process defined in (4.1). Then, for $t \leq s \leq T, x \in E$ and $h \in H$,

$$
\mathbb{E}\left[\nabla_{x} Y_{s}^{t, x} h\right]=\mathbb{E}\left[\int_{s}^{T} \psi\left(r, X_{r}^{t, x}, Y_{r}^{t, x}, Z_{r}^{t, x}\right) U_{r}^{h, t, x} d r\right]+\mathbb{E}\left[\phi\left(X_{T}^{t, x}\right) U_{T}^{h, t, x}\right] .
$$

Proof. We split the proof into two steps: we first prove the statement when $\psi$ is differentiable with respect to $x, y$ and $z$, and then we remove this additional assumption.

STEP 1 . We start by considering $\psi$ differentiable with respect to $x, y$ and $z$. For all $n \geq 1$, let us denote by $\left(X^{t, x}, Y^{n, t, x}, Z^{n, t, x}\right)$ the solution of the Markovian BSDE in (3.1) with final datum equal to $\phi_{n}$ in (3.6) in the place of $\phi$ :

$$
\left\{\begin{array}{l}
d Y_{\tau}^{n, t, x}=-\psi\left(\tau, X_{\tau}^{t, x}, Y_{\tau}^{n, t, x}, Z_{\tau}^{n, t, x}\right) d \tau+Z_{\tau}^{n, t, x} d W_{\tau}, \quad \tau \in[t, T] \\
Y_{T}^{n, t, x}=\phi_{n}\left(X_{T}^{t, x}\right) .
\end{array}\right.
$$

By estimate (3.30) in Corollary 3.11, for any $n \geq 1$, there exists a constant $C(n)$, depending on $n$, which is bounded for every $n$ and blows up as $n \rightarrow \infty$, and such that

$$
\left|Z_{s}^{n, t, x}\right|_{H} \leq C(n), \quad \mathbb{P} \text {-a.s., a.e. } s \in[t, T]
$$

In particular,

$$
\left|\psi\left(s, x, y, z_{1}\right)-\psi\left(s, x, y, z_{2}\right)\right| \leq C(n)\left|z_{1}-z_{2}\right|_{H}, \quad z_{1}, z_{2} \in H:\left|z_{i}\right|_{H} \leq C(n), i=1,2 .
$$

Therefore, the generator $\psi$ acts as a Lipschitz generator with respect to $z$ in the BSDE (5.2), so the Bismut-Elworthy formula stated in Theorem 4.4 holds true for the BSDE (5.2): for every $s \in[t, T]$,

$$
\mathbb{E}\left[\nabla_{x} Y_{s}^{n, t, x} h\right]=\mathbb{E}\left[\int_{s}^{T} \psi\left(r, X_{r}^{t, x}, Y_{r}^{n, t, x}, Z_{r}^{n, t, x}\right) U_{r}^{h, t, x} d r\right]+\mathbb{E}\left[\phi_{n}\left(X_{T}^{t, x}\right) U_{T}^{h, t, x}\right] .
$$

At this point we aim at taking the limit as $n \rightarrow \infty$ in (5.4).

We start by considering the right-hand side of (5.4). By the properties of the approximations $\left(\phi_{n}\right)_{n \geq 1}$ together with (4.2), by the dominated convergence theorem and the pointwise convergence of $\phi_{n}$ to $\phi$ we have

$$
\begin{aligned}
& \mathbb{E}\left[\left|\left[\phi_{n}\left(X_{T}^{t, x}\right)-\phi\left(X_{T}^{t, x}\right)\right] U_{T}^{h, t, x}\right|\right] \leq\left(\mathbb{E}\left[\left|\phi_{n}\left(X_{T}^{t, x}\right)-\phi\left(X_{T}^{t, x}\right)\right|^{2}\right]\right)^{1 / 2}\left(\mathbb{E}\left[\left|U_{T}^{h, t, x}\right|^{2}\right]\right)^{1 / 2} \\
& \leq C(T-t)^{-(1 / 2+\alpha)}\left(\mathbb{E}\left[\left|\phi_{n}\left(X_{T}^{t, x}\right)-\phi\left(X_{T}^{t, x}\right)\right|^{2}\right]\right)^{1 / 2} \rightarrow 0 \text { as } n \rightarrow \infty
\end{aligned}
$$

Therefore,

$$
\lim _{n \rightarrow \infty} \mathbb{E}\left[\phi_{n}\left(X_{T}^{t, x}\right) U_{T}^{h, t, x}\right]=\mathbb{E}\left[\phi\left(X_{T}^{t, x}\right) U_{T}^{h, t, x}\right]
$$


In order to compute the limit of the remaining term in the right-hand side of (5.4), we will show that

$$
\lim _{n \rightarrow \infty} \mathbb{E}\left[\int_{t}^{T}\left|\psi\left(r, X_{r}^{t, x}, Y_{r}^{n, t, x}, Z_{r}^{n, t, x}\right) U_{r}^{h, t, x}-\psi\left(r, X_{r}^{t, x}, Y_{r}^{t, x}, Z_{r}^{t, x}\right) U_{r}^{h, t, x}\right| d r\right]=0 .
$$

We notice that

$$
\begin{aligned}
& \mathbb{E}\left[\int_{t}^{T}\left|\psi\left(r, X_{r}^{t, x}, Y_{r}^{n, t, x}, Z_{r}^{n, t, x}\right) U_{r}^{h, t, x}-\psi\left(r, X_{r}^{t, x}, Y_{r}^{t, x}, Z_{r}^{t, x}\right) U_{r}^{h, t, x}\right| d r\right] \\
& =\mathbb{E}\left[\int_{t}^{\frac{t+T}{2}}\left|\psi\left(r, X_{r}^{t, x}, Y_{r}^{n, t, x}, Z_{r}^{n, t, x}\right) U_{r}^{h, t, x}-\psi\left(r, X_{r}^{t, x}, Y_{r}^{t, x}, Z_{r}^{t, x}\right) U_{r}^{h, t, x}\right| d r\right] \\
& +\mathbb{E}\left[\int_{\frac{t+T}{2}}^{T}\left|\psi\left(r, X_{r}^{t, x}, Y_{r}^{n, t, x}, Z_{r}^{n, t, x}\right) U_{r}^{h, t, x}-\psi\left(r, X_{r}^{t, x}, Y_{r}^{t, x}, Z_{r}^{t, x}\right) U_{r}^{h, t, x}\right| d r\right]=: I+I I .
\end{aligned}
$$

We start by estimating the term $I$. We have

$$
\begin{aligned}
I= & \mathbb{E}\left[\int_{t}^{\frac{t+T}{2}}\left|\psi\left(r, X_{r}^{t, x}, Y_{r}^{n, t, x}, Z_{r}^{n, t, x}\right) U_{r}^{h, t, x}-\psi\left(r, X_{r}^{t, x}, Y_{r}^{t, x}, Z_{r}^{t, x}\right) U_{r}^{h, t, x}\right| d r\right] \\
\leq & L_{\psi} \mathbb{E}\left[\int_{t}^{\frac{t+T}{2}}\left(\left|Y_{r}^{n, t, x}-Y_{r}^{t, x}\right|\left|U_{r}^{h, t, x}\right|\right) d r\right] \\
& +L_{\psi} \mathbb{E}\left[\int_{t}^{\frac{t+T}{2}}\left(\left|Z_{r}^{n, t, x}-Z_{r}^{t, x}\right|_{H}\left(1+\left|Z_{r}^{n, t, x}\right|_{H}+\left|Z_{r}^{t, x}\right|_{H}\right)\left|U_{r}^{h, t, x}\right|\right) d r\right]=: I_{a}+I_{b} .
\end{aligned}
$$

We recall that, by estimate (3.31) in Proposition 3.12, and since $\left\|\phi_{n}\right\|_{\infty} \leq K_{\phi}$, there exists a constant $C$, not depending on $n$, such that

$$
\left|Z_{t}^{n, t, x}\right|_{H} \leq C(T-t)^{-1 / 2}, \quad \mathbb{P} \text {-a.s. }
$$

So, since $Z_{r}^{n, t, x}=Z_{r}^{n, r, X_{r}^{t, x}}$ and $Z_{r}^{t, x}=Z_{r}^{r, X_{r}^{t, x}}$, for $r \in\left[t, \frac{t+T}{2}\right]$

$$
\left|Z_{r}^{n, t, x}\right|_{H}+\left|Z_{r}^{t, x}\right|_{H} \leq C \sup _{r \in\left[t, \frac{t+T}{2}\right]}(T-r)^{-1 / 2} \leq C(T-t)^{-1 / 2}, \quad \mathbb{P} \text {-a.s. }
$$

We only show the convergence of $I_{b}$ since the convergence of $I_{a}$ follows in a simpler way by the boundedness of $Y^{t, x}$ and of $Y^{n, t, x}$ (uniform in $n$ ), and by the convergence of $Y^{n, t, x}$ to $Y^{t, x}$ in $\mathscr{S}^{p}([t, T]), p \geq 2$. Using Hölder inequality with $p=\frac{2}{1-\beta}$ and $q=\frac{2}{1+\beta}$, for some $2 \alpha<\beta<1$, together with estimate (4.3) in Lemma 4.1, we get

$$
\begin{aligned}
I_{b} & \leq C(T-t)^{-\frac{1}{2}}(T-t)^{-\frac{1}{2} \beta} \mathbb{E}\left[\int_{t}^{\frac{t+T}{2}}\left|Z_{r}^{n, t, x}-Z_{r}^{t, x}\right|_{H}^{1-\beta}\left|U_{r}^{h, t, x}\right| d r\right] \\
& \leq C(T-t)^{-\frac{1}{2}}(T-t)^{-\frac{1}{2} \beta}\left(\mathbb{E}\left[\int_{t}^{\frac{t+T}{2}}\left|Z_{r}^{n, t, x}-Z_{r}^{t, x}\right|_{H}^{2} d r\right]\right)^{\frac{1-\beta}{2}}\left(\int_{t}^{\frac{t+T}{2}} \mathbb{E}\left[\mid U_{r}^{h, t, x} \frac{\frac{2}{1+\beta}}{1+\beta} d r\right)^{\frac{1+\beta}{2}}\right. \\
& \leq C(T-t)^{-\frac{1}{2}(1+\beta)}\left(\mathbb{E}\left[\int_{t}^{\frac{t+T}{2}}\left|Z_{r}^{n, t, x}-Z_{r}^{t, x}\right|_{H}^{2} d r\right]\right)^{\frac{1-\beta}{2}}\left(\int_{t}^{\frac{t+T}{2}} \frac{1}{(r-t)^{\frac{1+2 \alpha}{1+\beta}}} d r\right)^{\frac{1+\beta}{2}} \\
& \leq C(T-t)^{-\frac{1}{2}(1+\beta)}(T-t)^{\left(1-\frac{1+2 \alpha}{1+\beta}\right) \frac{1+\beta}{2}}\left(\mathbb{E}\left[\int_{t}^{\frac{t+T}{2}}\left|Z_{r}^{n, t, x}-Z_{r}^{t, x}\right|_{H}^{2} d r\right]\right)^{\frac{1-\beta}{2}} \\
& \leq C(T-t)^{-\frac{1}{2}-\alpha}\left(\mathbb{E}\left[\int_{t}^{\frac{t+T}{2}}\left|Z_{r}^{n, t, x}-Z_{r}^{t, x}\right|_{H}^{2} d r\right]\right)^{\frac{1-\beta}{2}} \rightarrow 0
\end{aligned}
$$


as $n \rightarrow \infty$, since $Z^{n, t, x} \rightarrow Z^{t, x}$ in $\mathscr{M}^{2}([t, T] ; H)$.

Let us now estimate the term $I I$. To this end, we recall that, by Theorem $3.3, Y^{n, t, x}, Y^{t, x}$ are bounded in $\mathscr{S}^{p}([t, T])$ and $Z^{n, t, x}, Z^{t, x}$ are bounded in $\mathscr{M}^{2 p}([t, T] ; H)$, by a constant independent on $n$. Moreover, by Proposition 3.6, $Y^{n, t, x}$ converges to $Y^{t, x}$ is $\mathscr{S}^{p}([t, T])$ and $Z^{n, t, x}$ converges to $Z^{t, x}$ in $\mathscr{M}^{2 p}([t, T] ; H)$, for any $p \geq 1$. By using again Hölder's inequality for some $p, q \geq 1, \frac{1}{p}+\frac{1}{q}=1$, and estimate (4.3) in Lemma 4.1, we get

$$
\begin{aligned}
I I & =\mathbb{E}\left[\int_{\frac{t+T}{2}}^{T}\left|\psi\left(r, X_{r}^{t, x}, Y_{r}^{n, t, x}, Z_{r}^{n, t, x}\right) U_{r}^{h, t, x}-\psi\left(r, X_{r}^{t, x}, Y_{r}^{t, x}, Z_{r}^{t, x}\right) U_{r}^{h, t, x}\right| d r\right] \\
& \leq \mathbb{E}\left[\sup _{s \in\left[\frac{t+T}{2}, T\right]}\left|U_{s}^{h, t, x}\right| \int_{\frac{t+T}{2}}^{T}\left|\psi\left(r, X_{r}^{t, x}, Y_{r}^{n, t, x}, Z_{r}^{n, t, x}\right)-\psi\left(r, X_{r}^{t, x}, Y_{r}^{t, x}, Z_{r}^{t, x}\right)\right| d r\right] \\
& \leq\left(\mathbb{E} \sup _{s \in\left[\frac{t+T}{2}, T\right]}\left|U_{s}^{h, t, x}\right|^{q}\right)^{\frac{1}{q}}\left(\mathbb{E}\left[\int_{\frac{t+T}{2}}^{T}\left|\psi\left(r, X_{r}^{t, x}, Y_{r}^{n, t, x}, Z_{r}^{n, t, x}\right)-\psi\left(r, X_{r}^{t, x}, Y_{r}^{t, x}, Z_{r}^{t, x}\right)\right| d r\right]^{p}\right)^{\frac{1}{p}} \\
& \leq C \frac{1}{(T-t)^{\frac{1}{2}+\alpha}}\left(\mathbb{E}\left[\int_{\frac{t+T}{2}}^{T}\left(\left|Y_{r}^{n, t, x}-Y_{r}^{t, x}\right|+\left|Z_{r}^{n, t, x}-Z_{r}^{t, x}\right|_{H}\left(1+\left|Z_{r}^{n, t, x}\right|_{H}+\left|Z_{r}^{t, x}\right|_{H}\right)\right) d r\right]^{p}\right)^{\frac{1}{p}} \\
& \leq C \frac{1}{(T-t)^{\frac{1}{2}+\alpha}}\left\{\frac{T-t}{2}\left(\mathbb{E}\left[\sup _{r \in[t, T]}\left|Y_{r}^{n, t, x}-Y_{r}^{t, x}\right|^{p}\right]\right)^{\frac{1}{p}}\right. \\
& \left.+\left(\mathbb{E}\left[\left(\int_{\frac{t+T}{2}}^{T}\left|Z_{r}^{n, t, x}-Z_{r}^{t, x}\right|_{H}^{2} d r\right)^{\frac{p}{2}}\left(\int_{\frac{t+T}{2}}^{T}\left(1+\left|Z_{r}^{n, t, x}\right|_{H}+\left|Z_{r}^{t, x}\right|_{H}\right)^{2} d r\right)^{\frac{p}{2}}\right]\right)^{\frac{1}{p}}\right\} \\
& \leq C \frac{1}{(T-t)^{\frac{1}{2}+\alpha}}\left\{\frac{T-t}{2}\left(\mathbb{E}\left[\sup _{r \in[t, T]}\left|Y_{r}^{n, t, x}-Y_{r}^{t, x}\right|^{p}\right]\right)^{\frac{1}{p}}\right. \\
& \left.+\left(\mathbb{E}\left[\int_{\frac{t+T}{2}}^{T}\left|Z_{r}^{n, t, x}-Z_{r}^{t, x}\right|_{H}^{2} d r\right]^{p}\right)^{\frac{1}{2 p}}\left(\mathbb{E}\left[\int_{\frac{t+T}{2}}^{T}\left(1+\left|Z_{r}^{n, t, x}\right|_{H}+\left|Z_{r}^{t, x}\right|_{H}\right)^{2} d r\right]^{p}\right)^{\frac{1}{2 p}}\right\} \rightarrow 0
\end{aligned}
$$

as $n \rightarrow \infty$. Collecting all the previous results, we deduce that, for every $s \in[t, T]$,

$$
\lim _{n \rightarrow \infty} \mathbb{E}\left[\nabla_{x} Y_{s}^{n, t, x} h\right]=\mathbb{E}\left[\int_{s}^{T} \psi\left(r, X_{r}^{t, x}, Y_{r}^{t, x}, Z_{r}^{t, x}\right) U_{r}^{h, t, x} d r\right]+\mathbb{E}\left[\phi\left(X_{T}^{t, x}\right) U_{T}^{h, t, x}\right] .
$$

In particular, by taking $s=t$ in $(5.6)$,

$$
\lim _{n \rightarrow \infty} \nabla_{x} Y_{t}^{n, t, x} h=\mathbb{E}\left[\int_{t}^{T} \psi\left(r, X_{r}^{t, x}, Y_{r}^{t, x}, Z_{r}^{t, x}\right) U_{r}^{h, t, x} d r\right]+\mathbb{E}\left[\phi\left(X_{T}^{t, x}\right) U_{T}^{h, t, x}\right],
$$

which shows that $\lim _{n \rightarrow \infty} \nabla_{x} Y_{t}^{n, t, x} h$ exists. Moreover, arguing as in the end of the proof of Theorem 4.1 in [21], we deduce that $\lim _{n \rightarrow \infty} \nabla_{x} Y_{t}^{n, t, x} h=\nabla_{x} Y_{t}^{t, x} h$ for all $h \in H$.

STEP 2. Let us now remove the differentiability assumptions on $\psi$. For any $k \geq 1$, let $\psi_{k}$ be the function defined in (3.7). From Lemma 3.5 we know that $\psi_{k}$ is differentiable and it preserves the Lipschitz constant, so that

$$
\left|\nabla_{x} \psi_{k}\right|_{E^{*}} \leq L_{\psi}, \quad\left|\nabla_{y} \psi_{k}\right| \leq L_{\psi}
$$

Moreover, from Lemma 3.5 we have $\psi_{k}(t, x, y, z) \rightarrow \psi(t, x, y, z)$ as $k \rightarrow+\infty$ for any $(t, x, y, z) \in[0, T] \times$ $E \times \mathbb{R} \times H$, and for any $t \in[0, T], x \in E, y \in \mathbb{R}, z_{1}, z_{2} \in H$,

$$
\left|\psi_{k}\left(t, x, y_{1}, z_{1}\right)-\psi_{k}\left(t, x, y_{2}, z_{2}\right)\right| \leq L_{\psi}\left(\left|y_{1}-y_{2}\right|+\left|z_{1}-z_{2}\right|_{H}\left(1+\left|z_{1}\right|_{H}+\left|z_{2}\right|_{H}\right)\right)
$$


for any $k \in \mathbb{N}$. We consider the BSDE with generator equal to $\psi_{k}$ in the place of $\psi$ :

$$
\left\{\begin{array}{l}
d Y_{\tau}^{k, t, x}=-\psi_{k}\left(\tau, X_{\tau}^{t, x}, Y_{\tau}^{k, t, x}, Z_{\tau}^{k, t, x}\right) d \tau+Z_{\tau}^{k, t, x} d W_{\tau}, \quad \tau \in[t, T], \\
Y_{T}^{k, t, x}=\phi\left(X_{T}^{t, x}\right) .
\end{array}\right.
$$

By the first part of the proof, for any $k \geq 1$,

$$
\mathbb{E}\left[\nabla_{x} Y_{s}^{k, t, x} h\right]=\mathbb{E}\left[\int_{s}^{T} \psi_{k}\left(r, X_{r}^{t, x}, Y_{r}^{k, t, x}, Z_{r}^{k, t, x}\right) U_{r}^{h, t, x} d r\right]+\mathbb{E}\left[\phi\left(X_{T}^{t, x}\right) U_{T}^{h, t, x}\right] .
$$

We aim at taking the limit as $k \rightarrow \infty$. We start by considering the first term in the right-hand side of (5.9), and we will show that

$$
\lim _{k \rightarrow \infty} \mathbb{E}\left[\int_{t}^{T}\left|\psi_{k}\left(r, X_{r}^{t, x}, Y_{r}^{k, t, x}, Z_{r}^{k, t, x}\right) U_{r}^{h, t, x}-\psi\left(r, X_{r}^{t, x}, Y_{r}^{t, x}, Z_{r}^{t, x}\right) U_{r}^{h, t, x}\right| d r\right]=0 .
$$

We start by splitting the integral above as follows:

$$
\begin{aligned}
& \mathbb{E}\left[\int_{t}^{T}\left|\psi_{k}\left(r, X_{r}^{t, x}, Y_{r}^{k, t, x}, Z_{r}^{k, t, x}\right) U_{r}^{h, t, x}-\psi\left(r, X_{r}^{t, x}, Y_{r}^{t, x}, Z_{r}^{t, x}\right) U_{r}^{h, t, x}\right| d r\right] \\
& =\mathbb{E}\left[\int_{t}^{\frac{t+T}{2}}\left|\psi_{k}\left(r, X_{r}^{t, x}, Y_{r}^{k, t, x}, Z_{r}^{k, t, x}\right) U_{r}^{h, t, x}-\psi\left(r, X_{r}^{t, x}, Y_{r}^{t, x}, Z_{r}^{t, x}\right) U_{r}^{h, t, x}\right| d r\right] \\
& +\mathbb{E}\left[\int_{\frac{t+T}{2}}^{T}\left|\psi_{k}\left(r, X_{r}^{t, x}, Y_{r}^{k, t, x}, Z_{r}^{k, t, x}\right) U_{r}^{h, t, x}-\psi\left(r, X_{r}^{t, x}, Y_{r}^{t, x}, Z_{r}^{t, x}\right) U_{r}^{h, t, x}\right| d r\right]=: I+I I
\end{aligned}
$$

In order to estimate the term $I$, we notice that

$$
\begin{aligned}
I & \leq \mathbb{E}\left[\int_{t}^{\frac{t+T}{2}}\left|\psi_{k}\left(r, X_{r}^{t, x}, Y_{r}^{k, t, x}, Z_{r}^{k, t, x}\right) U_{r}^{h, t, x}-\psi_{k}\left(r, X_{r}^{t, x}, Y_{r}^{t, x}, Z_{r}^{t, x}\right) U_{r}^{h, t, x}\right| d r\right] \\
& +\mathbb{E}\left[\int_{t}^{\frac{t+T}{2}}\left|\psi_{k}\left(r, X_{r}^{t, x}, Y_{r}^{t, x}, Z_{r}^{t, x}\right) U_{r}^{h, t, x}-\psi\left(r, X_{r}^{t, x}, Y_{r}^{t, x}, Z_{r}^{t, x}\right) U_{r}^{h, t, x}\right| d r\right]=: I_{a}+I_{b} .
\end{aligned}
$$

Concerning $I_{a}$, by (3.4)-(3.5) we can argue as for $I$ in Step 1, and get that $I_{a} \rightarrow 0$ as $k \rightarrow+\infty$. Let us now consider the term $I_{b}$. From Hypothesis 3.1 and formulas (3.4) and (3.5) it follows

$$
\left|\psi_{k}\left(r, X_{r}^{t, x}, Y_{r}^{t, x}, Z_{r}^{t, x}\right)-\psi\left(r, X_{r}^{t, x}, Y_{r}^{t, x}, Z_{r}^{t, x}\right)\right| \leq C\left(1+\left|Y_{r}^{t, x}\right|+\left|Z_{r}^{t, x}\right|_{H}^{2}\right),
$$

where $C$ is a positive constant depending on $L_{\psi}$ and $K_{\psi}$. Arguing as for $I$ is Step 1, it is possible to prove that

$$
r \mapsto\left(1+\left|Y_{r}^{t, x}\right|+\left|Z_{r}^{t, x}\right|_{H}^{2}\right)\left|U_{r}^{h, t, x}\right| \in L^{1}\left(\Omega ; L^{1}\left(t, \frac{t+T}{2} ; \mathbb{R}\right)\right) .
$$

On the other hand, recalling that $\psi_{k} \rightarrow \psi$ pointwise as $k \rightarrow+\infty$, we get that $I_{b} \rightarrow 0$ as $k \rightarrow+\infty$ by the dominated convergence theorem.

Let us now estimate $I I$. To this end, we notice that

$$
\begin{aligned}
I I \leq \mathbb{E} & {\left[\int_{\frac{t+T}{2}}^{T}\left|\psi_{k}\left(r, X_{r}^{t, x}, Y_{r}^{k, t, x}, Z_{r}^{k, t, x}\right) U_{r}^{h, t, x}-\psi_{k}\left(r, X_{r}^{t, x}, Y_{r}^{t, x}, Z_{r}^{t, x}\right) U_{r}^{h, t, x}\right| d r\right] } \\
& +\mathbb{E}\left[\int_{\frac{t+T}{2}}^{T}\left|\psi_{k}\left(r, X_{r}^{t, x}, Y_{r}^{t, x}, Z_{r}^{t, x}\right) U_{r}^{h, t, x}-\psi\left(r, X_{r}^{t, x}, Y_{r}^{t, x}, Z_{r}^{t, x}\right) U_{r}^{h, t, x}\right| d r\right]=: I I_{a}+I I_{b} .
\end{aligned}
$$


Arguing as for the term $I I$ in Step 1, we deduce that $I I_{a} \rightarrow 0$ as $k \rightarrow 0$. As far as $I I_{b}$ is considered, we get

$$
I I_{b} \leq\left(\mathbb{E}\left[\sup _{s \in\left[\frac{t+T}{2}, T\right]}\left|U_{s}^{h, t, x}\right|^{q}\right]\right)^{1 / q}\left(\mathbb{E}\left[\int_{\frac{t+T}{2}}^{T}\left|\psi_{k}\left(r, X_{r}^{t, x}, Y_{r}^{t, x}, Z_{r}^{t, x}\right)-\psi\left(r, X_{r}^{t, x}, Y_{r}^{t, x}, Z_{r}^{t, x}\right)\right| d r\right]^{p}\right)^{1 / p} .
$$

Arguing as for $I I$ in Step 1 it follows that

$$
r \mapsto\left(1+\left|Y_{r}^{t, x}\right|+\left|Z_{r}^{t, x}\right|_{H}^{2}\right)\left|U_{r}^{h, t, x}\right| \in L^{p}\left(\Omega ; L^{1}\left(\frac{t+T}{2}, T ; \mathbb{R}\right)\right) .
$$

Since $\psi_{k}$ pointwise converges to $\psi$, we can again apply the dominated convergence theorem which gives $I I_{b} \rightarrow 0$ as $k \rightarrow+\infty$. We can thus conclude that, for every $s \in[t, T]$,

$$
\lim _{k \rightarrow \infty} \mathbb{E}\left[\nabla_{x} Y_{s}^{k, t, x} h\right]=\mathbb{E}\left[\int_{s}^{T} \psi\left(r, X_{r}^{t, x}, Y_{r}^{t, x}, Z_{r}^{t, x}\right) U_{r}^{h, t, x} d r\right]+\mathbb{E}\left[\phi\left(X_{T}^{t, x}\right) U_{T}^{h, t, x}\right] .
$$

As in the end of Step 1, arguing as at the end of Theorem 4.1 in [21] we can show that, for any $s \in[t, T]$, $\lim _{k \rightarrow \infty} \mathbb{E}\left[\nabla_{x} Y_{s}^{k, t, x} h\right]=\mathbb{E}\left[\nabla_{x} Y_{s}^{t, x} h\right]$.

We now state two corollaries: the former is about integral estimates of $\nabla_{x} Y^{t, x}$, the latter is about the identification of $\nabla_{x} Y^{t, x}$ with $Z^{t, x}$ without differentiability assumptions. Notice that, by means of the Bismut formula (5.1), we can also recover estimate (3.32) on $\nabla_{x} Y^{t, x}$.

Corollary 5.2. Let $(t, x) \in[0, T] \times E$. Under the assumptions of Theorem 5.1, the process $\nabla_{x} Y^{t, x}$ belongs to $\mathscr{M}^{2}([t, T])$, and there exists a constant $C$ depending only on $L_{\psi}, K_{\psi}, K_{\phi}$ such that

$$
\mathbb{E}\left[\int_{t}^{T}\left|\nabla_{x} Y_{s}^{t, x}\right|^{2} d s\right] \leq C(T-t)^{-2 \alpha}
$$

Proof. Integrating (5.1) between $t$ and $T$ we get

$$
\begin{aligned}
& \int_{t}^{T}\left|\mathbb{E}\left[\nabla_{x} Y_{s}^{t, x}\right]\right|^{2} d s=\int_{t}^{T}\left|\mathbb{E}\left[\int_{s}^{T} \psi\left(r, X_{r}^{t, x}, Y_{r}^{t, x}, Z_{r}^{t, x}\right) U_{r}^{h, t, x} d r\right]+\mathbb{E}\left[\phi\left(X_{T}^{t, x}\right) U_{T}^{h, t, x}\right]\right|^{2} d s \\
& \leq C \int_{t}^{T}\left|\mathbb{E}\left[\int_{s}^{T} \psi\left(r, X_{r}^{t, x}, Y_{r}^{t, x}, Z_{r}^{t, x}\right) U_{r}^{h, t, x} d r\right]\right|^{2} d s+\int_{t}^{T}\left|\mathbb{E}\left[\phi\left(X_{T}^{t, x}\right) U_{T}^{h, t, x}\right]\right|^{2} d s=: I+I I .
\end{aligned}
$$

We have

$$
I I \leq \int_{t}^{T}\|\phi\|_{\infty} \frac{1}{(T-t)^{1+2 \alpha}} d r=C(T-t)^{-2 \alpha} .
$$

For what concerns $I$, we split it as

$$
\begin{aligned}
I & =C\left(\int_{\frac{t+T}{2}}^{T}\left|\mathbb{E}\left[\int_{s}^{T} \psi\left(r, X_{r}^{t, x}, Y_{r}^{t, x}, Z_{r}^{t, x}\right) U_{r}^{h, t, x} d r\right]\right|^{2} d s\right. \\
& \left.+\int_{t}^{\frac{t+T}{2}}\left|\mathbb{E}\left[\int_{s}^{T} \psi\left(r, X_{r}^{t, x}, Y_{r}^{t, x}, Z_{r}^{t, x}\right) U_{r}^{h, t, x} d r\right]\right|^{2} d s\right)=: I_{a}+I_{b} .
\end{aligned}
$$

From (4.3) and Proposition 3.3 we have

$$
I_{a} \leq C \int_{\frac{t+T}{2}}^{T}\left(\mathbb{E}\left[\sup _{r \in\left[\frac{t+T}{2}, T\right]}\left|U_{r}^{h, t, x}\right| \int_{s}^{T}\left|\psi\left(r, X_{r}^{t, x}, Y_{r}^{t, x}, Z_{r}^{t, x}\right)\right| d r\right]\right)^{2} d s
$$




$$
\begin{aligned}
& \leq C \int_{\frac{t+T}{2}}^{T} \mathbb{E}\left[\sup _{r \in\left[\frac{t+T}{2}, T\right]}\left|U_{r}^{h, t, x}\right|^{2}\right] \mathbb{E}\left[\left(\int_{s}^{T}\left(1+\left|Y_{r}^{t, x}\right|+\left|Z_{r}^{t, x}\right|_{H}^{2}\right) d r\right)^{2}\right] d s \\
& \leq C(T-t)^{-1-2 \alpha} \int_{\frac{t+T}{2}}^{T} \mathbb{E}\left[\left(\int_{s}^{T}\left(1+\left|Y_{r}^{t, x}\right|+\left|Z_{r}^{t, x}\right|_{H}^{2}\right) d r\right)^{2}\right] d s \leq C(T-t)^{-2 \alpha} .
\end{aligned}
$$

On the other hand, we consider the function under the integral sign in $I_{b}$ and we split it as follows:

$$
\begin{aligned}
& \mathbb{E}\left[\int_{s}^{T} \psi\left(r, X_{r}^{t, x}, Y_{r}^{t, x}, Z_{r}^{t, x}\right) U_{r}^{h, t, x} d r\right] \\
& =\mathbb{E}\left[\int_{s}^{\frac{t+T}{2}} \psi\left(r, X_{r}^{t, x}, Y_{r}^{t, x}, Z_{r}^{t, x}\right) U_{r}^{h, t, x} d r\right]+\mathbb{E}\left[\int_{\frac{t+T}{2}}^{T} \psi\left(r, X_{r}^{t, x}, Y_{r}^{t, x}, Z_{r}^{t, x}\right) U_{r}^{h, t, x} d r\right] \\
& =: I_{b}^{\prime}+I_{b}^{\prime \prime} .
\end{aligned}
$$

We argue as in the proof of Theorem 5.1, Step 1. In particular, arguing as for the estimate of $I$ we infer that $\left|I_{b}^{\prime}\right| \leq C(T-t)^{-1 / 2-\alpha}$ for some positive constant $C$. On the other hand, as far as $I_{b}^{\prime \prime}$ is considered, arguing as in the estimate of $I I$, we get that $\left|I_{b}^{\prime \prime}\right| \leq C(T-t)^{-1 / 2-\alpha}$ for some positive constant $C$. Hence,

$$
I_{b} \leq C \int_{t}^{\frac{t+T}{2}}(T-t)^{-1-2 \alpha} d s=C(T-t)^{-2 \alpha},
$$

and this concludes the proof.

In the following we prove that the identification of $Z$ with the directional derivative of $Y$ remains true also when $\phi$ and $\psi$ are not differentiable.

Corollary 5.3. Under the assumptions of Theorem 5.1, for every $(t, x) \in[0, T] \times E$,

$$
Z_{t}^{t, x}=\nabla_{x} Y_{t}^{t, x}(-A)^{-\alpha} .
$$

Proof. Let $\phi$ and $\psi$ be respectively approximated by $\phi_{n}$ and $\psi_{n}$ in (3.6) and (3.7), and let $\left(Y^{n, t, x}, Z^{n, t, x}\right)$ be the solution of the BSDE with final datum $\phi_{n}$ and generator $\psi_{n}$. By Theorem 4.6 we already know that $Z_{t}^{n, t, x}=\nabla_{x} Y_{t}^{n, t, x}(-A)^{-\alpha}$. On the other hand, we have shown in Theorem 5.1 that $x \mapsto Y_{\tau}^{t, x}=$ $v\left(\tau, X_{\tau}^{t, x}\right)$ is differentiable and that $\nabla_{x} Y_{\tau}^{n, t, x} \rightarrow \nabla_{x} Y_{\tau}^{t, x}, d t \otimes d \mathbb{P}$ a.e. and a.s., as $n \rightarrow \infty$. Moreover, by computing the joint quadratic variation between the process $v^{n}\left(\tau, X_{\tau}^{t, x}\right):=Y_{\tau}^{n, t, x}, t \leq \tau \leq T$, and $\int_{t}^{\cdot} \xi_{s} d W_{s}, \xi \in \mathscr{M}^{2}([t, T] ; H)$, it turns out that

$$
\int_{t}^{\tau} \nabla v^{n}\left(s, X_{s}^{t, x}\right)(-A)^{-\alpha} \xi_{s} d s=\int_{t}^{\tau} Z_{s}^{n, t, x} \xi_{s} d s, \mathbb{P} \text {-a.s., a.e. } \tau \in[t, T] .
$$

By taking a subsequence (that for simplicity we call again $n$ ) and letting $n \rightarrow \infty$ in both sides, from Proposition 3.6 we get

$$
\int_{t}^{\tau} \nabla v\left(s, X_{s}^{t, x}\right)(-A)^{-\alpha} \xi_{s} d s=\int_{t}^{\tau} Z_{s}^{t, x} \xi_{s} d s, \text { a.e. } \tau \in[t, T], \mathbb{P} \text {-a.s. },
$$

which gives formula (5.12).

Using Theorem 3.10, we can give an existence and uniqueness result for the Kolmogorov equation (4.16) and we can provide a Feynman-Kac formula in the quadratic case and in the Banach framework. 
Theorem 5.4. Let Hypotheses 2.1 and 3.1 hold true. Then there exists a unique mild solution $v(t, x)$ of the semilinear Kolmogorov equation (4.16) given by the formula

$$
v(t, x)=Y_{t}^{t, x},
$$

where $\left(X^{t, x}, Y^{t, x}, Z^{t, x}\right)$ is the solution to the FBSDE (3.1), and $\mathbb{P}$-a.s.,

$$
Y_{s}^{t, x}=v\left(s, X_{s}^{t, x}\right), \quad Z_{s}^{t, x}=\nabla_{x} v\left(s, X_{s}^{t, x}\right) \nabla_{x} X_{s}^{t, x}(-A)^{-\alpha} \text {, a.e. } s \in[t, T] .
$$

In particular,

$$
|v(t, x)| \leq C, \quad\left|\nabla_{x} v(t, x)\right| \leq C(T-t)^{-\left(\frac{1}{2}+\alpha\right)} .
$$

If in addition $\phi$ is Gâteaux differentiable with bounded derivative, and $\psi$ is Gâteaux differentiable with respect to $x, y$ and $z$, then

$$
\left|Z_{s}^{t, x}\right|_{H} \leq C
$$

Proof. For the first part without differentiability assumptions on $\phi$ and $\psi$, it is enough to apply Theorem 5.1 and Corollary 5.3 to get existence of the solution, as well as the estimate for $v$. The uniqueness follows from the uniqueness of the solution of the related BSDE. The estimate for $\nabla_{x} v(t, x)$ is a direct consequence of Proposition 3.12. The second part of the result can be proved in a standard way by means of Proposition 3.7 and the identification of $Z$ proved in Theorem 3.10, see e.g. the proof of Theorem 6.2 in [11].

\section{A quadratic optimal control problem}

In this section we deal with the controlled state equation

$$
\left\{\begin{array}{l}
d X_{\tau}^{u}=A X_{\tau}^{u} d \tau+F\left(X_{\tau}^{u}\right) d \tau+Q u_{\tau} d \tau+(-A)^{-\alpha} d W_{\tau}, \quad \tau \in[t, T], \\
X_{t}^{u}=x \in E,
\end{array}\right.
$$

where $Q=(-A)^{-\alpha}$ or $Q=I$, and $u$ is the control process belonging to a suitable space $\mathscr{U}$ of $H$-valued functions. We will study the optimal control problem associated to equation (6.1) with cost functional $J:[0, T] \times E \times \mathscr{U} \rightarrow \mathbb{R}$ defined by

$$
J(t, x, u):=\mathbb{E}\left[\int_{t}^{T} \ell\left(s, X_{s}^{u}, u_{s}\right) d s\right]+\mathbb{E}\left[\Phi\left(X_{T}^{u}\right)\right],
$$

that we are going to minimize over all admissible controls. We define the value function of the optimal control problem as

$$
V(t, x):=\inf _{u \in \mathscr{U}} J(t, x, u), \quad x \in H, t \in[0, T] .
$$

For any $p \geq 1$, we introduce the spaces of admissible control processes

$$
\begin{aligned}
\mathscr{U}_{p} & :=\left\{u \in L^{2}\left(\Omega ; L^{p}(0, T ; H)\right): u \text { is adapted }\right\}, \\
\mathscr{U}_{p}^{\alpha} & :=\left\{u \in L^{2}\left(\Omega ; L^{p}\left(0, T ; D\left((-A)^{\alpha}\right)\right)\right): u \text { is adapted }\right\},
\end{aligned}
$$

where $\left.D\left((-A)^{\alpha}\right)\right)$ is endowed with the norm

$$
|x|_{\alpha}:=|x|_{H}+\left|(-A)^{\alpha} x\right|_{H} .
$$

We first prove some results about well posedness of the controlled equation (6.1). The main novelty towards Section 2 and the known results in the literature is that the controls $u$ are not necessarily bounded, together with the fact that $X$ evolves in a Banach space E.

Beside Hypothesis 2.1 we assume the following. 
Hypothesis 6.1. There exists $\beta>0$ such that $D\left((-A)^{\beta}\right) \subset E$ with continuous embedding.

Remark 6.2. Let $A$ be an operator satisfying Hypothesis 2.1-(i). If Hypothesis 6.1 holds true, then we have the following.

(i) For any $t>0$ and $h \in H, e^{t A} h \in E$ and there exists a positive constant $c$ such that

$$
\left|e^{t A} h\right|_{E} \leq c t^{-\beta}|h|_{H}
$$

(ii) For any $t>0$ and $h \in H$, there exists a positive constant $c$ such that

$$
\left|e^{t A}(-A)^{-\alpha} h\right|_{E} \leq c t^{(-\beta+\alpha) \wedge 0}|h|_{H}
$$

(iii) For any $t>0$ and $h \in D\left((-A)^{\alpha}\right)$, there exists a positive constant $c$ such that

$$
\left|e^{t A} h\right|_{E}=\left|e^{t A}(-A)^{-\alpha}(-A)^{\alpha} h\right|_{E} \leq c t^{(-\beta+\alpha) \wedge 0}\left|(-A)^{\alpha} h\right|_{H} \leq c t^{(-\beta+\alpha) \wedge 0}|h|_{\alpha} .
$$

Remark 6.3. Hypothesis 6.1 may be replaced by the weaker condition in Remark 6.2-(i). However, this condition would not imply Remark 6.2-(ii)-(iii).

Example 6.4. Let $\mathscr{D} \subset \mathbb{R}^{2}$ be a bounded domain with smooth boundary. Set $H=L^{2}(\mathscr{D}), E=C(\overline{\mathscr{D}})$, and let $A$ be the Laplace operator with Dirichlet boundary conditions. Then, Hypothesis 6.1 is satisfied with $\beta>1 / 2$.

We will deal with mild solutions to (6.1), namely adapted processes $X^{t, x, u}:[t, T] \times \Omega \rightarrow E$ such that

$$
X_{\tau}^{t, x, u}=e^{\tau A} x+\int_{t}^{\tau} e^{(\tau-s) A} F\left(X_{s}^{t, x, u}\right) d s+\int_{t}^{\tau} e^{(\tau-s) A} Q u_{s} d s+\int_{t}^{\tau} e^{(\tau-s) A}(-A)^{-\alpha} d W_{s}
$$

for any $\tau \in[t, T], \mathbb{P}$-a.s. For any $t \in[0, T], u \in \mathscr{U}$, we set

$$
I^{u}(t, \tau):=\int_{t}^{\tau} e^{(\tau-s) A} Q u_{s} d s, \quad \tau \in[t, T] .
$$

Lemma 6.5. Let $A$ be an operator satisfying Hypothesis 2.1-(i), and assume that Hypothesis 6.1 holds true for some positive constant $\beta$. Let $p \geq 1$, and set $p^{\prime}$ be the conjugate exponent of $p$, i.e., $p^{-1}+\left(p^{\prime}\right)^{-1}=1$. Then the following hold.

(i) Case $Q=(-A)^{-\alpha}$ and $p^{\prime}[(\beta-\alpha) \vee 0]<1$.

For any $u \in \mathscr{U}_{p}, I^{u}(t, \tau) \in E$ for any $\tau \in[t, T], \mathbb{P}$-a.s., and there exists a positive constant $c_{\alpha, \beta, p, T}$ such that

$$
\left|I^{u}(t, \tau)\right|_{E} \leq c_{\alpha, \beta, p, T}\|u\|_{L^{p}(0, T ; H)}, \quad \tau \in[t, T], \mathbb{P} \text {-a.s. }
$$

(ii) Case $Q=I$ and $p^{\prime}[(\beta-\alpha) \vee 0]<1$.

For any $u \in \mathscr{U}_{p}^{\alpha}, I^{u}(t, \tau) \in E$ for any $\tau \in[t, T], \mathbb{P}$-a.s., and there exists a positive constant $c_{\alpha, \beta, p, T}$ such that

$$
\left|I^{u}(t, \tau)\right|_{E} \leq c_{\alpha, \beta, p, T}\|u\|_{L^{p}\left(0, T ; D\left((-A)^{\alpha}\right)\right)}, \quad \tau \in[t, T], \mathbb{P} \text {-a.s. }
$$

(iii) Case $Q=I$ and $p^{\prime} \beta<1$.

For any $u \in \mathscr{U}_{p}, I^{u}(t, \tau) \in E$ for any $\tau \in[t, T], \mathbb{P}$-a.s., and $I^{u}(t, \tau)$ satisfies estimate (6.8) for some positive constant $c_{\alpha, \beta, p, T}$. 
Proof. Let us prove item (i), items (ii) and (iii) follow from similar arguments. From Hypothesis 6.1, we have

$$
\left|e^{(\tau-s) A}(-A)^{-\alpha} u_{s}\right|_{E} \leq c(\tau-s)^{(-\beta+\alpha) \wedge 0}\left|u_{s}\right|_{H} \quad \text { a.e. } s \in(t, \tau), \mathbb{P} \text {-a.s. }
$$

Therefore,

$$
\begin{aligned}
\left|\int_{t}^{\tau} e^{(\tau-s) A}(-A)^{-\alpha} u_{s} d s\right|_{E} & \leq \int_{t}^{\tau}\left|e^{(\tau-s) A}(-A)^{-\alpha} u_{s}\right|_{E} d s \leq c \int_{t}^{\tau}(\tau-s)^{(-\beta+\alpha) \wedge 0}\left|u_{s}\right|_{H} d s \\
& \leq c\left(\int_{t}^{\tau}(\tau-s)^{[(-\beta+\alpha) \wedge 0] p^{\prime}} d s\right)^{1 / p^{\prime}}\|u\|_{L^{p}(0, T ; H)} \\
& \leq c(\tau-t)^{(-\beta+\alpha) \wedge 0+1 / p^{\prime}}\|u\|_{L^{p}(0, T ; H)}, \quad \mathbb{P} \text {-a.s. }
\end{aligned}
$$

Thanks to Lemma 6.5, arguing as in [6, Theorem 7.11] we deduce the following result, which is the counterpart of Proposition 2.5-(i) for the controlled equation.

Proposition 6.6. Let Hypothesis 2.1 holds true, and assume that Hypothesis 6.1 holds true for some positive constant $\beta$. Let $t \in[0, T], p \geq 1$, and set $p^{\prime}$ be the conjugate exponent of $p$. Then the following hold.

(i) Case $Q=(-A)^{-\alpha}, p^{\prime}[(\beta-\alpha) \vee 0]<1$.

For any $x \in E$ and $u \in \mathscr{U}_{p}$, there exists a unique mild solution $X_{\tau}^{t, x, u}$ to (6.1) belonging to $\mathscr{S}^{2}((t, T] ; E)$. Moreover, there exists a positive constant $c$ such that, for any $\tau \in[t, T]$,

$$
\left|X_{\tau}^{t, x, u}\right|_{E} \leq c\left(|x|_{E}+\|u\|_{L^{p}(t, T ; H)}^{2 m+1}+\sup _{\tau \in[t, T]}\left|w^{A}(t, \tau)\right|_{E}^{2 m+1}\right), \quad \mathbb{P} \text {-a.s. }
$$

(ii) Case $Q=I, p^{\prime}[(\beta-\alpha) \vee 0]<1$.

For any $x \in E$ and $u \in \mathscr{U}_{p}^{\alpha}$, there exists a unique mild solution $X_{\tau}^{t, x, u}$ to (6.1) belonging to $\mathscr{S}^{2}((t, T] ; E)$. Moreover, there exists a positive constant $c$ such that, for any $\tau \in[t, T]$,

$$
\left|X_{\tau}^{t, x, u}\right|_{E} \leq c\left(|x|_{E}+\|u\|_{L^{p}\left(t, T ; D\left((-A)^{\alpha}\right)\right)}^{2 m+1}+\sup _{\tau \in[t, T]}\left|w^{A}(t, \tau)\right|_{E}^{2 m+1}\right), \quad \mathbb{P} \text {-a.s. }
$$

(iii) Case $Q=I, p^{\prime} \beta<1$.

For any $x \in E$ and $u \in \mathscr{U}_{p}$, there exists a unique mild solution $X_{\tau}^{t, x, u}$ to (6.1) belonging to $\mathscr{S}^{2}((t, T] ; E)$. Moreover, there exists a positive constant $c$ such that, for any $\tau \in[t, T]$,

$$
\left|X_{\tau}^{t, x, u}\right|_{E} \leq c\left(|x|_{E}+\|u\|_{L^{p}(t, T ; H)}^{2 m+1}+\sup _{\tau \in[t, T]}\left|w^{A}(t, \tau)\right|_{E}^{2 m+1}\right), \quad \mathbb{P} \text {-a.s. }
$$

Proof. We show item $(i)$, the proof of items $(i i)$ and (iii) being analogous. Since by Lemma 6.5 the convolution defined in (6.7) is a well defined $E$-valued process for any $u \in \mathscr{U}_{p}$, it is possible to argue as in [6, Theorem 7.11]. Therefore, by applying the fixed point theorem we infer that for any $t \in[0, T], x \in E$ and $u \in \mathscr{U}_{p}$, there exists a unique mild solution $X^{\alpha, t, x, u}$ to (6.1) with $F$ replaced by its Yosida approximations $F_{\alpha}, \alpha>0$, such that $X^{\alpha, t, x, u}$ satisfies (6.9). Further, the sequence $\left\{X^{\alpha, t, x, u}\right\}_{\alpha>0}$ converges as $\alpha \rightarrow 0$ to the mild solution $X^{t, x, u}$ to (6.1). In particular, estimate (6.9) holds true also for $X^{t, x, u}$. 


\subsection{The structure condition: the case $Q=(-A)^{-\alpha}$}

In this section we deal with control processes $u \in \mathscr{U}_{2}$, and with the controlled equation

$$
\left\{\begin{array}{l}
d X_{\tau}^{u}=A X_{\tau}^{u} d \tau+F\left(X_{\tau}^{u}\right) d \tau+(-A)^{-\alpha} u_{\tau} d \tau+(-A)^{-\alpha} d W_{\tau}, \quad \tau \in[t, T] \\
X_{t}^{u}=x \in E
\end{array}\right.
$$

satisfying the so called structure condition: the control affects the system only through the noise.

We make the following assumptions on the cost functional (6.2).

Hypothesis 6.7. Let $\phi: E \rightarrow \mathbb{R}$ and $\ell:[0, T] \times E \times H \rightarrow \mathbb{R}$ be two measurable functions satisfying the following properties.

(i) $\phi$ is continuous and bounded.

(ii) For all $t \in[0, T], u \in H$, the function $x \mapsto \ell(t, x, u)$ is bounded and continuous from $E$ onto $\mathbb{R}$. For all $t \in[0, T], x \in E$, the function $u \mapsto \ell(t, x, u)$ is continuous from $H$ onto $\mathbb{R}$. Further, there exist $c, C, R$ positive constants such that, for all $t \in[0, T], x \in E, u \in H$,

$$
\begin{aligned}
& 0 \leq \ell(t, x, u) \leq c\left(1+|u|_{H}\right)^{2}, \\
& \ell(t, x, u) \geq C|u|_{H}^{2}, \quad|u|_{H} \geq R .
\end{aligned}
$$

(iii) There exists a positive constant $L>0$ such that, for all $t \in[0, T], x_{1}, x_{2} \in E, u \in H$,

$$
\left|\ell\left(t, x_{1}, u\right)-\ell\left(t, x_{2}, u\right)\right| \leq L\left|x_{1}-x_{2}\right|_{E}
$$

Remark 6.8. Under Hypothesis 6.7-(ii), it is easy to see that there exist $c, R$ positive constants such that

$$
\ell(t, x, u) \geq c\left(|u|_{H}^{2}-R^{2}\right), \quad t \in[0, T], x \in E, u \in H .
$$

We introduce the Hamiltonian function

$$
\psi(t, x, z):=\inf _{u \in H}\left\{\ell(t, x, u)+\langle z, u\rangle_{H}\right\}, \quad t \in[0, T], x \in E, z \in H .
$$

Arguing as in [13, Lemma 3.1] we deduce an analogous result.

Lemma 6.9. Let Hypotheses 6.7 be satisfied. Then, the function $\psi$ in (6.15) is Borel measurable, and there exists a positive constant $C$ such that

$$
-C\left(1+|z|_{H}^{2}\right) \leq \psi(t, x, z) \leq \ell(t, x, u)+|z|_{H}|u|_{H}, \quad t \in[0, T], x \in E, z, u \in H .
$$

Further, if the minimum in (6.15) is attained, it is attained in a ball of radius $C\left(1+|z|_{H}\right)$, i.e.,

$$
\begin{aligned}
& \psi(t, x, z)=\inf _{u \in H,|u|_{H} \leq C\left(1+|z|_{H}\right)}\left\{\ell(t, x, u)+\langle z, u\rangle_{H}\right\}, \quad t \in[0, T], x \in E, z \in H, \\
& \psi(t, x, z) \leq \ell(t, x, u)+\langle z, u\rangle_{H}, \quad|u|_{H} \geq C\left(1+|z|_{H}\right) .
\end{aligned}
$$

Finally, there exists a positive constant $C$ such that, for any $x_{1}, x_{2} \in E, z_{1}, z_{2} \in H$,

$$
\left|\psi\left(t, x_{1}, z_{1}\right)-\psi\left(t, x_{2}, z_{2}\right)\right| \leq C\left(\left|x_{1}-x_{2}\right|_{E}+\left|z_{1}-z_{2}\right|_{H}\left(1+\left|z_{1}\right|_{H}+\left|z_{2}\right|_{H}\right)\right), \quad t \in[0, T] .
$$


The HJB equation associated to the control problem (6.3), related to the controlled state equation (6.12), is given by

$$
\left\{\begin{array}{l}
\frac{\partial v}{\partial t}(t, x)=-\mathscr{L} v(t, x)+\psi\left(t, x, v(t, x), \nabla^{(-A)^{-\alpha}} v(t, x)\right), \quad t \in[0, T], x \in E, \\
v(T, x)=\phi(x)
\end{array}\right.
$$

where $\psi$ is defined in (6.15). The HJB equation (6.19) turns out to be a semilinear Kolmogorv equation as (4.16), with $\psi$ and $\phi$ satisfying Hypotehsis 3.1. So by Theorem 5.4 its mild solution can be represented in terms of the solution $\left(X^{t, x}, Y^{t, x}, Z^{t, x}\right)$ of the forward-backward system (3.1).

In the following Theorem we state and prove the fundamental relation, and we characterize the optimal control with a feedback law.

Theorem 6.10. Let Hypotheses 2.1, 6.7 hold true, and assume that Hypothesis 6.1 holds true with a constant $\beta$ such that $\beta-\alpha<1 / 2$. Let $X^{t, x, u}$ be the mild solution of $(6.12), V(t, x)$ be the value function of the control problem (6.3), and $v$ be the mild solution of the HJB equation (6.19). Then, for any $(t, x) \in[0, T] \times E$ and $u \in \mathscr{U}_{2}$, the so called fundamental relation holds true:

$$
v(t, x)=J(t, x, u)+\mathbb{E}\left[\int_{\tau}^{T}\left(\psi\left(s, X_{s}^{t, x, u}, Z_{s}^{t, x}\right)-\ell\left(s, X_{s}^{t, x, u}, Z_{s}^{t, x}\right)-Z_{s}^{t, x} u_{s}\right) d s\right] .
$$

In particular, $v(t, x) \leq V(t, x)$, for all $(t, x) \in[0, T] \times E$. Moreover, if there exists a measurable function $\gamma:[0, T] \times E \times H \rightarrow H$ satisfying

$$
\psi(t, x, z)=\ell(t, x, \gamma(t, x, z))+\langle z, \gamma(t, x, z)\rangle_{H}, \quad t \in[0, T], x \in E, z \in H,
$$

then

$$
v(t, x)=V(t, x)
$$

and, thanks to (6.17), the process $\bar{u}$ defined by

$$
\bar{u}_{s}:=\gamma\left(s, X_{s}^{x, \bar{u}}, \nabla_{x} v\left(s, X_{s}^{x, \bar{u}}\right) \nabla_{x} X_{s}^{x, \bar{u}}(-A)^{-\alpha}\right) \quad \text { for -a.e. } s \in(0, T), \quad \mathbb{P} \text {-a.s., }
$$

belongs to $\mathscr{U}_{2}$ and it is optimal.

Proof. The proof is standard and follows the same lines of [13, Proposition 4.1]. We notice that, by Proposition 6.6-(i), problem (6.12) admits a unique mild solution $X^{t, x, u}$ for any $u \in \mathscr{U}_{2}$. Further, for any $u \in \mathscr{U}_{2}$, we introduce the family of stopping times $\tau_{n}$ defined by

$$
\tau_{n}:=\inf \left\{\tau \in[t, T]: \int_{t}^{\tau}\left|u_{s}\right|_{H}^{2} d s>n\right\}, \quad n \in \mathbb{N} .
$$

Then we proceed as in [13, Proposition 4.1], by applying the Girsanov Theorem and using the fact that $\psi$ satisfies Hypothesis 3.1-(ii), and that the pair of processes $\left(Y^{t, x}, Z^{t, x}\right)$, solution to the Markovian BSDE in (3.1), are identified respectively with the solution $v$ of the HJB equation (6.19) and with its directional derivative $\nabla^{(-A)^{-\alpha}} v$. Namely, by Theorems 5.1 and $5.4, Y_{s}^{t, x}=v\left(s, X_{s}^{t, x}\right)$ and $Z_{s}^{t, x}=$ $\nabla_{x} v\left(s, X_{s}^{x, \bar{u}}\right) \nabla_{x} X_{s}^{x, \bar{u}}(-A)^{-\alpha}$.

\subsection{The case $Q=I$ with a special running cost}

In the present section we deal with control processes $u \in \mathscr{U}_{2}$, and with the controlled equation

$$
\left\{\begin{array}{l}
d X_{\tau}^{u}=A X_{\tau}^{u} d \tau+F\left(X_{\tau}^{u}\right) d \tau+u_{\tau} d \tau+(-A)^{-\alpha} d W_{\tau}, \quad \tau \in[t, T] \\
X_{t}^{u}=x \in E
\end{array}\right.
$$

The controlled equation (6.20) has a different structure towards (6.12) considered in Subsection 6.1, so the problem is different, and we need different assumptions on the cost functional (6.2). 
Hypothesis 6.11. Let $\phi: E \rightarrow \mathbb{R}$ and $\ell:[0, T] \times E \times H \rightarrow \mathbb{R} \cup\{+\infty\}$ be two measurable functions satisfying the following properties.

(i) $\phi$ is continuous and bounded.

(ii) For all $t \in[0, T], u \in D\left((-A)^{\alpha}\right)$, the function $x \mapsto \ell(t, x, u)$ is bounded and continuous from $E$ onto $\mathbb{R}$. For all $t \in[0, T], x \in E$, the function $u \mapsto \ell(t, x, u)$ is continuous from $D\left((-A)^{\alpha}\right)$ onto $\mathbb{R}$. Further, there exists $c, C, R$ positive constants such that, for all $t \in[0, T], x \in E$ and $u \in D\left((-A)^{\alpha}\right)$,

$$
\begin{aligned}
& 0 \leq \ell(t, x, u) \leq c\left(1+|u|_{\alpha}\right)^{2}, \\
& \ell(t, x, u) \geq C|u|_{\alpha}^{2}, \quad|u|_{\alpha} \geq R .
\end{aligned}
$$

(iii) There exists a positive constant $L>0$ such that, for any $t \in[0, T], u \in D\left((-A)^{\alpha}\right), x_{1}, x_{2} \in E$,

$$
\left|\ell\left(t, x_{1}, u\right)-\ell\left(t, x_{2}, u\right)\right| \leq L\left|x_{1}-x_{2}\right|_{E} .
$$

Remark 6.12. Condition (6.22) in Hypothesis 6.11 implies that, if $u$ does not take values in $D\left((-A)^{\alpha}\right)$, then $J(t, x, u)=+\infty$. In particular, $\inf _{u \in \mathscr{U}_{2}^{\alpha}} J(t, x, u)=\inf _{u \in \mathscr{U}_{2}} J(t, x, u)$, so we can limit ourselves to consider here the space of admissible controls $\mathscr{U}_{2}^{\alpha}$.

Remark 6.13. Under Hypothesis 6.11-(ii), there exist positive constants $c, R$ such that, for any $t \in[0, T]$, $x \in E, u \in D\left((-A)^{\alpha}\right)$, we have $\ell(t, x, u) \geq c\left(|u|_{\alpha}^{2}-R^{2}\right)$.

We introduce the Hamiltonian function

$$
\psi^{\alpha}(t, x, z):=\inf _{u \in D\left((-A)^{\alpha}\right)}\left\{\ell(t, x, u)+\left\langle z,(-A)^{\alpha} u\right\rangle_{H}\right\}, \quad t \in[0, T], x \in E, z \in H .
$$

Arguing again as in [13, Lemma 3.1], we infer the following properties of $\psi^{\alpha}$.

Lemma 6.14. Let Hypotheses 6.11 be satisfied. Then, the function $\psi^{\alpha}$ in (6.23) is Borel measurable and there exists a positive constant $C$ such that

$$
-C\left(1+|z|_{H}^{2}\right) \leq \psi^{\alpha}(t, x, z) \leq \ell(t, x, u)+|z|_{H}|u|_{\alpha}, \quad t \in[0, T], x \in E, z \in H, u \in D\left((-A)^{\alpha}\right) .
$$

Further, if the minimum in (6.23) is attained, it is attained in a ball of radius $C\left(1+|z|_{H}\right)$, i.e.,

$$
\psi^{\alpha}(t, x, z)=\inf _{u \in D\left((-A)^{\alpha}\right),|u|_{\alpha} \leq C\left(1+|z|_{H}\right)}\left\{\ell(t, x, u)+z(-A)^{\alpha} u\right\}, \quad t \in[0, T], x \in E, z \in H .
$$

Finally, for any $x_{1}, x_{2} \in E, z_{1}, z_{2} \in H, \psi^{\alpha}$, there exists a positive constant $C$ such that

$$
\left|\psi^{\alpha}\left(t, x_{1}, z_{1}\right)-\psi^{\alpha}\left(t, x_{2}, z_{2}\right)\right| \leq C\left(\left|x_{1}-x_{2}\right|_{E}+\left|z_{1}-z_{2}\right|_{H}\left(1+\left|z_{1}\right|_{H}+\left|z_{2}\right|_{H}\right)\right), \quad t \in[0, T] .
$$

The HJB equation associated to the control problem (6.3), related to the controlled state equation (6.20), is given by

$$
\left\{\begin{array}{l}
\frac{\partial v}{\partial t}(t, x)=-\mathscr{L} v(t, x)+\psi^{\alpha}\left(t, x, v(t, x), \nabla^{(-A)^{-\alpha}} v(t, x)\right), \quad t \in[0, T], x \in E, \\
v(T, x)=\phi(x),
\end{array}\right.
$$

where $\psi^{\alpha}$ is defined in (6.23). Again, the HJB equation (6.27) turns out to be a semilinear Kolmogorv equation as (4.16), with $\psi^{\alpha}$ and $\phi$ satisfying Hypotehsis 3.1. So by Theorem 5.4 its mild solution can be represented in terms of the solution $\left(X^{t, x}, Y^{t, x}, Z^{t, x}\right)$ of the forward-backward system

$$
\begin{cases}d X_{\tau}=A X_{\tau} d \tau+F\left(X_{\tau}\right) d \tau+(-A)^{-\alpha} d W_{\tau}, & \tau \in[t, T], \\ X_{t}=x & \\ d Y_{\tau}=-\psi^{\alpha}\left(\tau, X_{\tau}, Y_{\tau}, Z_{\tau}\right) d \tau+Z_{\tau} d W_{\tau}, & \tau \in[t, T] \\ Y_{T}=\phi\left(X_{T}\right) & \end{cases}
$$


which is nothing else than the forward-backward system (3.1) with $\psi$ instead of $\psi^{\alpha}$.

As in Subsection 6.1, in the following Theorem we state and prove the fundamental relation, and we characterize the optimal control with a feedback law.

Theorem 6.15. Let Hypotheses 2.1, 6.11 hold true, and assume that Hypothesis 6.1 holds true with a constant $\beta$ such that $\beta-\alpha<1 / 2$. Let $X^{t, x, u}$ be the mild solution of $(6.20), V(t, x)$ be the value function of the control problem (6.3), and $v$ be the mild solution of the HJB equation (6.27). Then, for any $(t, x) \in[0, T] \times E$ and $u \in \mathscr{U}_{2}^{\alpha}$,

$$
v(t, x)=J(t, x, u)+\mathbb{E} \int_{\tau}^{T}\left(\psi^{\alpha}\left(s, X_{s}^{t, x, u}, Z_{s}^{t, x}\right)-\ell\left(s, X_{s}^{t, x, u}, Z_{s}^{t, x}\right)-Z_{s}^{t, x}(-A)^{\alpha} u_{s}\right) d s .
$$

In particular, $v(t, x) \leq V(t, x)$, for all $t \in[0, T], x \in E$. Moreover, if there exists a measurable function $\gamma^{\alpha}:[0, T] \times E \times H \rightarrow D\left((-A)^{\alpha}\right)$ satisfying

$$
\psi^{\alpha}(t, x, z)=\ell\left(t, x, \gamma^{\alpha}(t, x, z)\right)+\left\langle z,(-A)^{\alpha} \gamma^{\alpha}(t, x, z)\right\rangle_{H}, \quad t \in[0, T], x \in E, z \in H,
$$

then

$$
v(t, x)=V(t, x)
$$

and, thanks to (6.25), the process

$$
\bar{u}_{s}^{\alpha}:=\gamma^{\alpha}\left(s, X_{s}^{x, \bar{u}^{\alpha}}, \nabla_{x} v\left(s, X_{s}^{x, \bar{u}^{\alpha}}\right) \nabla_{x} X_{s}^{x, \bar{u}^{\alpha}}(-A)^{-\alpha}\right) \quad \text { for-a.e. } s \in(0, T), \quad \mathbb{P} \text {-a.s. },
$$

belongs to $\mathscr{U}_{2}^{\alpha}$ and it is optimal.

Proof. Notice that by Proposition 6.6-(ii), for any $u \in \mathscr{U}_{2}^{\alpha}$ there exists a unique mild solution $X^{t, x, u}$ to (6.20) which satisfies (6.10). The proof is similar to the one of Theorem 6.10. The main difference consists in the fact that, for any given $u \in \mathscr{U}_{2}^{\alpha}$, we introduce a family of stopping times depending on the norm $|\cdot|_{\alpha}$ :

$$
\tau_{n}:=\inf \left\{\tau \in[t, T]: \int_{t}^{\tau}\left|u_{s}\right|_{\alpha}^{2} d s>n\right\}, \quad n \in \mathbb{N} .
$$

Then, we set $u_{\tau}^{n}:=u_{\tau} \mathbb{1}_{\tau \leq \tau_{n}}+u_{0} \mathbb{1}_{\tau>\tau_{n}}, u_{0} \in D\left((-A)^{\alpha}\right)$, and we introduce the process

$$
W_{\tau}^{n}:=W_{\tau}+\int_{t}^{\tau}(-A)^{\alpha} u_{s}^{n} d s
$$

Afterwards, we apply the Girsanov Theorem: writing $u_{s}=(-A)^{-\alpha}(-A)^{\alpha} u_{s}$ in (6.20), we get that $X^{t, x, u^{n}}$ is mild solution to

$$
\left\{\begin{array}{l}
d X_{\tau}=A X_{\tau} d \tau+F\left(X_{\tau}\right) d \tau+(-A)^{-\alpha} d W_{\tau}^{n}, \quad \tau \in[t, T] \\
X_{t}=x \in E
\end{array}\right.
$$

By (6.26) in Lemma 6.14, we see that Hypothesis 3.1-(ii) is verified by $\psi^{\alpha}$. We conclude by arguing again as in [13, Proposition 4.1] and in Theorem 6.10.

\subsection{The case $Q=I$ with a general running cost}

In this subsection we deal with the general controlled equation (6.20) under Hypothesis 6.7 on the coefficients of the cost functional, and we consider control processes $u \in \mathscr{U}_{2}$. Unlike the two cases just treated, in this framework the HJB equation would not have the structure of equation (4.16) since the Hamiltonian function would depend on $\nabla v$, not only on the directional derivative $\nabla^{(-A)^{-\alpha}} v$, see e.g. [9], 
formula (6.67) and the discussion related to formulas (4.278)-(4.279). Up to our knowledge, when $\phi$ in only continuous, the well posedness of such an equation is an open problem: in [3] an equation of this type is solved in mild sense with Lipschitz type assumptions on the final datum $\phi$.

For this reason, we will not end up identifying the value function (6.3) with the solution of the HJB equation, but instead we will approximate it. The following result will be used in the aforementioned approximation of the value function.

Proposition 6.16. Assume that Hypothesis 2.1 holds true. Let $t \in[0, T], x \in E$ and $u, \tilde{u} \in \mathscr{U}_{2}$. Then,

$$
\sup _{\tau \in[t, T]}\left|X_{\tau}^{t, x, u}-X_{\tau}^{t, x, \tilde{u}}\right|_{H}^{2} \leq \int_{t}^{T}\left|u_{s}-\tilde{u}_{s}\right|_{H}^{2} d s, \quad \mathbb{P} \text {-a.s. }
$$

where $X^{t, x, u}$ and $X^{t, x, \tilde{u}}$ are respectively the mild solutions to (6.20) with control $u$ and $\tilde{u}$.

Proof. Let us set $L(\tau):=X_{\tau}^{t, x, u}-X_{\tau}^{t, x, \tilde{u}}$ and let us assume that $L(\tau)$ is a strict solution to

$$
\left\{\begin{array}{l}
\frac{d}{d \tau} L(\tau)=A L(\tau)+F\left(X_{\tau}^{t, x, u}\right)-F\left(X_{\tau}^{t, y, \tilde{u}}\right)+u_{\tau}-\tilde{u}_{\tau}, \quad \tau \in[t, T], \\
L(t)=0,
\end{array}\right.
$$

otherwise we can use an approximation argument as in the proof of Proposition 2.5(ii). Then, the nonpositivity of $A$, the dissipativity of $F$, the Cauchy-Schwartz inequality and the Young inequality give

$$
\frac{1}{2} \frac{d}{d s}|L(s)|_{H}^{2} \leq\left\langle u_{s}-\tilde{u}_{s}, L(s)\right\rangle_{H} \leq \frac{1}{2}\left|u_{s}-\tilde{u}_{s}\right|_{H}^{2}+\frac{1}{2}|L(s)|_{H}^{2}, \quad s \in[t, T], \quad \mathbb{P} \text {-a.s. }
$$

Integrating between $t$ and $\tau$ and applying the Gronwall Lemma, we get

$$
|L(\tau)|_{H}^{2} \leq \int_{t}^{\tau}\left|u_{s}-\tilde{u}_{s}\right|_{H}^{2} d s, \quad \forall \tau \in[t, T], \quad \mathbb{P} \text {-a.s. }
$$

and we immediately deduce (6.30).

Thanks to Proposition 6.16 we deduce that, up to a subsequence, we can approximate $X^{t, x, u}$ in $H$ by means of mild solutions $X^{t, x, u^{n}}$ of problem (6.20), with $u$ replaced by $u^{n}$, where $\left(u_{n}\right) \subset \mathscr{U}_{p}$ satisfies $u^{n} \rightarrow u$ in $\mathscr{U}_{p}$. In the following Proposition we prove that a similar approximation holds true in $E$.

Proposition 6.17. Let Hypothesis 2.1 holds true. Let $t \in[0, T], p \geq 2$, and set $p^{\prime}$ be the conjugate exponent of $p$. Assume that Hypothesis 6.1 holds true for some positive constant $\beta$ such that $p^{\prime} \beta<1$. Let $u \in \mathscr{U}_{p}$ and $\left(u^{n}\right) \subset \mathscr{U}_{p}$ be such that $u^{n} \rightarrow u$ in $\mathscr{U}_{p}$. Then, for any $x \in E$,

$$
\lim _{n \rightarrow+\infty}\left|X_{\tau}^{t, x, u^{k_{n}}}-X_{\tau}^{t, x, u}\right|_{E}=0, \quad \forall \tau \in[t, T], \quad \mathbb{P} \text {-a.s. }
$$

where $\left(u^{k_{n}}\right) \subset\left(u^{n}\right)$ be such that $u_{s}^{k_{n}} \rightarrow u_{s} \mathbb{P}$ - a.s. for a.e. $s \in(t, T)$.

Proof. As usual, we limit ourselves to consider the case $t=0$. For any $n \in \mathbb{N}$, let us set $L^{n}:=$ $X^{x, u^{k_{n}}}-X^{x, u}$, where $X^{x, u^{k_{n}}}$ and $X^{x, u}$ are mild solutions to (6.20) with initial datum $x$ and control processes $u^{k_{n}}$ and $u$, respectively. Further, let us denote by $N$ the subset of $\Omega$ such that $\mathbb{P}(N)=0$ and $u_{s}^{k_{n}} \rightarrow u_{s}$ on $\Omega \backslash N$ for a.e. $s \in(t, T)$. Then, for any $t \in[0, T]$

$$
L_{t}^{n}=\int_{0}^{t} e^{(t-s) A}\left(F\left(X_{s}^{x, u^{k_{n}}}\right)-F\left(X_{s}^{x, u}\right)\right) d s+\int_{0}^{t} e^{(t-s) A}\left(u_{s}^{k_{n}}-u_{s}\right) d s, \quad \mathbb{P} \text {-a.s. }
$$


which gives

$$
\left|L_{t}^{n}\right|_{E} \leq \int_{0}^{t}\left|e^{(t-s) A}\left(F\left(X_{s}^{x, u^{k_{n}}}\right)-F\left(X_{s}^{x, u}\right)\right)\right|_{E} d s+\int_{0}^{t}\left|e^{(t-s) A}\left(u_{s}^{k_{n}}-u_{s}\right)\right|_{E} d s=: I_{1}^{n}(t)+I_{2}^{n}(t), \quad \mathbb{P} \text {-a.s. }
$$

Let us estimate $I_{1}^{n}$ and $I_{2}^{n}$ separately. As far as $I_{1}^{n}$ is concerned, from the boundedness of $e^{t A}$ on $E$, Hypothesis 2.1-4. and (6.11), it follows that

$$
\left|e^{(t-s) A}\left(F\left(X_{s}^{x, u^{k_{n}}}\right)-F\left(X_{s}^{x, u}\right)\right)\right|_{E}<\infty, \quad s \in(0, T),
$$

on $\Omega \backslash N$. Further, from (6.4) it follows that

$$
\left|e^{(t-s) A}\left(F\left(X_{s}^{x, u^{k_{n}}}\right)-F\left(X_{s}^{x, u}\right)\right)\right|_{E} \leq c(t-s)^{-\beta}\left|F\left(X_{s}^{x, u^{k_{n}}}\right)-F\left(X_{s}^{x, u}\right)\right|_{H},
$$

on $\Omega \backslash N$, for any $s \in(0, T)$. Since $F$ is continuous on $H$, from (6.30) we infer that $\mid F\left(X_{s}^{x, u^{n}}\right)-$ $\left.F\left(X_{s}^{x, u}\right)\right|_{H} \rightarrow 0$ on $\Omega \backslash N$ as $n \rightarrow+\infty$ for any $s \in(0, T)$. The dominated convergence theorem implies that $I_{1}^{n} \rightarrow 0$ as $n \rightarrow+\infty$ on $\Omega \backslash N$.

Concerning $I_{2}^{n}$, from (6.4) and arguing as above we get

$$
I_{2}^{n}(t) \leq c \int_{0}^{t}(t-s)^{-\beta}\left|u_{s}^{k_{n}}-u_{s}\right|_{H} d s \leq c T^{-\beta+1 / p^{\prime}}\left\|u^{k_{n}}-u\right\|_{L^{p}(0, T ; H)} \rightarrow 0, \quad n \rightarrow+\infty,
$$

on $\Omega \backslash N$. This concludes the proof.

\subsubsection{The approximate optimal control problem}

We will consider the Hamiltonian function $\psi^{\alpha}$ in (6.23) under Hypothesis 6.7. This prevents us to obtain directly estimates as those in Lemmas 6.9 and 6.14 , since we don't have the structure condition and the assumptions on $\ell$ are not sufficient to bound the term $(-A)^{\alpha} u$. For this reason, for any $n \in \mathbb{N}$ we introduce the function $\ell_{n}:[0, T] \times E \times H \longrightarrow \mathbb{R}$ defined by

$$
\ell_{n}(s, x, u):=\ell(s, x, u)+\frac{1}{n}\left|(-A)^{\alpha} u\right|_{H}^{2} .
$$

Lemma 6.18. Let $A$ be an operator satisfying Hypothesis 2.1-(i). Then the function $\ell_{n}$ in (6.32) satisfies the following conditions: for any $(t, x) \in[0, T] \times H$,

$$
\begin{aligned}
& 0 \leq \ell_{n}(t, x, u) \leq c_{n}\left(1+|u|_{\alpha}^{2}\right) \quad u \in D\left((-A)^{\alpha}\right), \\
& \exists c_{n}, R>0: \ell_{n}(t, x, u) \geq c_{n}|u|_{\alpha}^{2}-c R^{2}, \quad u \in D\left((-A)^{\alpha}\right) .
\end{aligned}
$$

Proof. The first inequality directly comes from (6.32). On the other hand, for any $t \in[0, T], x \in E$ and $u \in D\left((-A)^{\alpha}\right)$, by Remark 6.8 we have

$$
\ell_{n}(t, x, u) \geq c\left(|u|_{H}^{2}-R^{2}\right)+\frac{1}{n}\left|(-A)^{\alpha} u\right|_{H}^{2} \geq c_{n}|u|_{\alpha}^{2}-c R^{2} .
$$

For any $n \in \mathbb{N}$, we introduce the approximate Hamiltonian function

$$
\psi_{n}(t, x, z):=\inf _{u \in D\left((-A)^{\alpha}\right)}\left\{\ell_{n}(t, x, u)+\left\langle z,(-A)^{\alpha} u\right\rangle_{H}\right\}, \quad t \in[0, T], x \in E, z \in H .
$$

Estimates in Lemma 6.18 give the following result, which is analogous to Lemma 6.14. 
Lemma 6.19. Let Hypothesis 6.7 be satisfied, and let $A$ be an operator satisfying Hypothesis 2.1-(i). Then, for any $n \in \mathbb{N}$, the function $\psi_{n}$ in (6.33) is Borel measurable, and there exists a positive constant $C_{n}$ such that

$$
-C_{n}\left(1+|z|_{H}^{2}\right) \leq \psi_{n}(t, x, z) \leq \ell_{n}(t, x, u)+|z|_{H}|u|_{\alpha}, \quad t \in[0, T], x \in E, z \in H, u \in D\left((-A)^{\alpha}\right) .
$$

Further, if the minimum in (6.33) is attained, it is attained in a ball of radius $C_{n}\left(1+|z|_{H}\right)$, i.e.,

$$
\psi_{n}(t, x, z)=\inf _{u \in D\left((-A)^{\alpha}\right),|u|_{\alpha} \leq C_{n}\left(1+|z|_{H}\right)}\left\{\ell_{n}(t, x, u)+z(-A)^{\alpha} u\right\}, \quad t \in[0, T], x \in E, z \in H .
$$

In particular, there exists a positive constant $C_{n}$ such that, for any $x_{1}, x_{2} \in E, z_{1}, z_{2} \in H$,

$$
\left|\psi_{n}\left(t, x_{1}, z_{1}\right)-\psi_{n}\left(t, x_{2}, z_{2}\right)\right| \leq C_{n}\left(\left|x_{1}-x_{2}\right|_{E}+\left|z_{1}-z_{2}\right|_{H}\left(1+\left|z_{1}\right|_{H}+\left|z_{2}\right|_{H}\right)\right), \quad t \in[0, T] .
$$

For any $n \in \mathbb{N}$, we introduce the approximate cost functional defined by

$$
J_{n}(t, x, u):=\mathbb{E}\left[\int_{t}^{T} \ell_{n}\left(s, X_{s}^{u}, u_{s}\right) d s\right]+\mathbb{E}\left[\Phi\left(X_{T}^{u}\right)\right]
$$

and the associated approximated optimal control problem

$$
V_{n}(t, x):=\inf _{u \in \mathscr{U}_{2}^{\alpha}} J_{n}(t, x, u), \quad t \in[0, T], x \in E .
$$

The HJB equation associated to the control problem (6.37), related to the controlled state equation (6.20), is given by

$$
\left\{\begin{array}{l}
\frac{\partial v}{\partial t}(t, x)=-\mathscr{L} v(t, x)+\psi^{n}\left(t, x, v(t, x), \nabla^{(-A)^{-\alpha}} v(t, x)\right), \quad t \in[0, T], x \in E, \\
v(T, x)=\phi(x)
\end{array}\right.
$$

where $\psi^{n}$ is defined in (6.33). The HJB equation (6.38) is the analogous of (6.27) in Section 6.2. So again by Theorem 5.4 , its solution can be represented in terms of the solution $\left(X^{t, x}, Y^{n, t, x}, Z^{n t, x}\right)$ of the forward-backward system

$$
\begin{cases}d Y_{\tau}^{n, t, x}=-\psi_{n}\left(\tau, X_{\tau}^{t, x}, Z_{\tau}^{n, t, x}\right) d \tau+Z_{\tau}^{n, t, x} d W_{\tau}, & \tau \in[t, T], \\ Y_{T}^{n, t, x}=\Phi\left(X_{T}^{t, x}\right) & \\ d X_{\tau}^{t, x}=A X_{\tau}^{t, x} d \tau+F\left(X_{\tau}^{t, x}\right) d \tau+(-A)^{-\alpha} d W_{\tau}, & \tau \in[t, T], \\ X_{t}^{t, x}=x \in E & \end{cases}
$$

which is nothing else than the forward-backward system (6.28) with $\psi^{n}$ instead of $\psi^{\alpha}$.

We consider the following assumptions.

Hypothesis 6.20. For any $n \in \mathbb{N}$, there exists a measurable function $\gamma_{n}:[0, T] \times E \times H \rightarrow D\left((-A)^{\alpha}\right)$ satisfying

$$
\psi_{n}(t, x, z)=\ell_{n}\left(t, x, \gamma_{n}(t, x, z)\right)+\left\langle z,(-A)^{\alpha} \gamma_{n}(t, x, z)\right\rangle_{H}, \quad t \in[0, T], x \in E, z \in H .
$$

We state now the analogous of Theorem 6.15 for the approximate optimal control problems (6.37).

Theorem 6.21. Let Hypotheses 2.1, 6.7 hold true, and assume that Hypothesis 6.1 holds true with $\beta<\frac{1}{2}$. Let $X^{u, t, x}$ be the solution of equation (6.20) and for any $n \in \mathbb{N}$, let $V_{n}$ be the function defined in (6.37), and $v_{n}$ be the mild solution of the HJB equation (6.38). Then, for any $(t, x) \in[0, T] \times E$ and $u \in \mathscr{U}_{2}^{\alpha}$,

$$
v_{n}(t, x)=J_{n}(t, x, u)+\mathbb{E}\left[\int_{\tau}^{T}\left(\psi_{n}\left(s, X_{s}^{t, x, u}, Z_{s}^{n, t, x}\right)-\ell_{n}\left(s, X_{s}^{t, x, u}, Z_{s}^{n, t, x}\right)-Z_{s}^{n, t, x}(-A)^{\alpha} u_{s}\right) d s\right]
$$


where $\left(X^{t, x}, Y^{n, t, x}, Z^{n, t, x}\right)$ is the solution to (6.39). In particular, $v_{n}(t, x) \leq V_{n}(t, x)$, for all $(t, x) \in$ $[0, T] \times E$. Finally, if Hypothesis 6.20 holds true, then

$$
v_{n}(t, x)=V_{n}(t, x)
$$

and, thanks to (6.25), the process

$$
\bar{u}_{s}^{n}:=\gamma_{n}\left(s, X_{s}^{x, \bar{u}^{n}}, \nabla_{x} v\left(s, X_{s}^{x, \bar{u}^{n}}\right) \nabla_{x} X_{s}^{x, \bar{u}^{n}}(-A)^{-\alpha}\right) \quad \text { for -a.e. } s \in(0, T), \quad \mathbb{P} \text {-a.s. }
$$

belongs to $\mathscr{U}_{2}^{\alpha}$ and it is optimal.

\subsubsection{A characterization of the value function}

In the present section we show that the value function $V$ of the optimal control problem (6.3) can be approximated by the sequence $\left(v_{n}\right)$ of mild solutions to $(6.38)$, that are identified with the approximated value functions $\left(V_{n}\right)$, see formula (6.41) in Theorem 6.21. As a byproduct, we deduce that the sequence $\left(\bar{u}^{n}\right)$ defined in (6.42) is a minimizing sequence for (6.3), and it is a bounded sequence in $\mathscr{U}_{2}$.

We start by introducing the Yosida approximations of $u \in \mathscr{U}_{2}$, namely a suitable sequence $\left(u_{k}\right)_{k \geq 1} \subset$ $\mathscr{U}_{2}^{\alpha}$ which converges to $u$ in $\mathscr{U}_{2}$. Since $\bar{u}^{n} \in \mathscr{U}_{2}^{\alpha}$ for any $n \in \mathbb{N}$, this would allow to approximate $V(t, x)$ in terms of $J\left(t, x, \bar{u}^{n}\right)$.

Definition 6.22. For any $\varepsilon>0$,

(i) we denote by $u_{\varepsilon} \in \mathscr{U}_{2}$ any admissible control such that $J\left(t, x, u_{\varepsilon}\right) \leq V(t, x)+\varepsilon$.

(ii) we denote by $u_{\varepsilon, k}$ the Yosida approximations of $u_{\varepsilon}$, i.e.,

$$
u_{\varepsilon, k}(t, \omega):= \begin{cases}k R(k, A)\left(u_{\varepsilon}(t, \omega)\right), & \text { if } u_{\varepsilon}(t, \omega) \text { is well defined } \\ 0 & \text { otherwise. }\end{cases}
$$

Lemma 6.23. Let $A$ be an operator satisfying Hypothesis 2.1-(i). Let $\varepsilon>0$ and $u_{\varepsilon}, u_{\varepsilon, k}$, with $k \in \mathbb{N}$, be the processes introduced in Definition 6.22. Then, for any $k \in \mathbb{N}, \delta \in[0,1]$ and $(t, \omega) \in[0, T] \times \Omega$,

$$
\begin{aligned}
& \left|u_{\varepsilon, k}(t, \omega)\right|_{H} \leq C\left|u_{\varepsilon}(t, \omega)\right|_{H} \\
& \left|(-A)^{\delta} u_{\varepsilon, k}(t, \omega)\right|_{H} \leq c_{\delta} k^{\delta}\left|u_{\varepsilon}(t, \omega)\right|_{H}
\end{aligned}
$$

for some positive constants $C, c_{\delta}$ not depending neither on $k$ nor on $u_{\varepsilon}$. In particular, $u_{\varepsilon, k} \in \mathscr{U}_{2}^{\delta}$, $u_{\varepsilon, k} \rightarrow u_{\varepsilon} \mathbb{P}$-a.s., a.e. in $[t, T]$ as $k \rightarrow+\infty$, and

$$
u_{\varepsilon, k} \rightarrow u_{\varepsilon} \text { in } \mathscr{U}_{2} \quad \text { as } n \rightarrow+\infty .
$$

Proof. Estimate (6.43) directly follows from the properties of $R(k, A)$. Further, the fact that $u_{\varepsilon, k} \rightarrow u_{\varepsilon}$ $\mathbb{P}$-a.s., a.e. in $[t, T]$ as $k \rightarrow+\infty$ follows from the properties of Yosida approximations. Then, convergence (6.45) follows from the dominated convergence theorem, Finally, it easily follows that

$$
\left|(-A) u_{\varepsilon, k}(t, \omega)\right|_{H} \leq k C\left|u_{\varepsilon}(t, \omega)\right|_{H},
$$

for any $(t, \omega) \in[0, T] \times \Omega$ and any $k \in \mathbb{N}$, where $C$ is the same positive constant as in (6.43). Interpolation estimates give (6.44).

Proposition 6.24. Let Hypotheses 2.1, 6.7, 6.1 hold true. Let $\varepsilon>0$ and let $u_{\varepsilon}, u_{\varepsilon, n}$, with $n \in \mathbb{N}$, be the processes introduced in Definition 6.22, and let $J, J_{n}$ be respectively the cost functionals in $(6.2),(6.36)$. Then for any $(t, x) \in[0, T] \times E$ we have

$$
J_{n}\left(t, x, u_{\varepsilon, n}\right) \rightarrow J\left(t, x, u_{\varepsilon}\right), \quad n \rightarrow+\infty .
$$


Proof. Since $u_{\varepsilon, n}$ pointwise converges to $u$, a.e. in $(0, T), \mathbb{P}$-a.s., from (6.45) in Lemma 6.23 and Proposition 6.17 it follows that $X_{\tau}^{t, x, u_{\varepsilon, n}} \rightarrow X_{\tau}^{t, x, u_{\varepsilon}} \mathbb{P}$-a.s. in $E$ as $n \rightarrow+\infty$ for any $\tau \in[t, T]$. By dominated convergence theorem we deduce that

$$
\mathbb{E}\left[\Phi\left(X_{T}^{u_{\varepsilon, n}}\right)\right] \rightarrow \mathbb{E}\left[\Phi\left(X_{T}^{u_{\varepsilon}}\right)\right], \quad n \rightarrow+\infty .
$$

To estimate the convergence of the approximate running $\operatorname{cost} \ell_{n}$ in (6.32), we consider separately the two terms in (6.32). We stress that

$$
\begin{aligned}
& \mathbb{E}\left[\int_{t}^{T}\left|\ell\left(s, X_{s}^{u_{\varepsilon, n}},\left(u_{\varepsilon, n}\right)_{s}\right)-\ell\left(s, X_{s}^{u_{\varepsilon}},\left(u_{\varepsilon}\right)_{s}\right)\right| d s\right] \\
& \leq \mathbb{E}\left[\int_{t}^{T}\left|\ell\left(s, X_{s}^{u_{\varepsilon, n}},\left(u_{\varepsilon, n}\right)_{s}\right)-\ell\left(s, X_{s}^{u_{\varepsilon}},\left(u_{\varepsilon, n}\right)_{s}\right)\right| d s\right]+\mathbb{E}\left[\int_{t}^{T}\left|\ell\left(s, X_{s}^{u_{\varepsilon}},\left(u_{\varepsilon, n}\right)_{s}\right)-\ell\left(s, X_{s}^{u_{\varepsilon}},\left(u_{\varepsilon}\right)_{s}\right)\right| d s\right] .
\end{aligned}
$$

Arguing as above, from Hypothesis 6.7-(iii) and (6.31) we get

$$
\left|\ell\left(s, X_{s}^{u_{\varepsilon, n}},\left(u_{\varepsilon, n}\right)_{s}\right)-\ell\left(s, X_{s}^{u_{\varepsilon}},\left(u_{\varepsilon, n}\right)_{s}\right)\right| \leq L\left|X_{s}^{u_{\varepsilon, n}}-X_{s}^{u_{\varepsilon}}\right|_{E} \rightarrow 0, \quad n \rightarrow+\infty, \quad \mathbb{P} \text {-a.s. }
$$

Further, from (6.13) in Hypothesis 6.7-(ii) and (6.43) we infer that

$$
\left|\ell\left(s, X_{s}^{u_{\varepsilon, n}},\left(u_{\varepsilon, n}\right)_{s}\right)-\ell\left(s, X_{s}^{u_{\varepsilon, n}},\left(u_{\varepsilon}\right)_{s}\right)\right| \leq c\left(1+\left|\left(u_{\varepsilon}\right)_{s}\right|_{H}^{2}\right),
$$

for any $s \in(t, T), \mathbb{P}$-a.s. By dominated convergence theorem we get

$$
\mathbb{E}\left[\int_{t}^{T}\left|\ell\left(s, X_{s}^{u_{\varepsilon}, n},\left(u_{\varepsilon, n}\right)_{s}\right)-\ell\left(s, X_{s}^{u_{\varepsilon}},\left(u_{\varepsilon, n}\right)_{s}\right)\right| d s\right] \rightarrow 0, \quad n \rightarrow+\infty .
$$

Moreover, the continuity of $\ell$ with respect to $u$ and the dominated convergence theorem give

$$
\int_{t}^{T}\left|\ell\left(s, X_{s}^{u_{\varepsilon}},\left(u_{\varepsilon, n}\right)_{s}\right)-\ell\left(s, X_{s}^{u_{\varepsilon}},\left(u_{\varepsilon}\right)_{s}\right)\right| d s \rightarrow 0, n \rightarrow+\infty .
$$

Finally, since $\alpha \in(0,1 / 2)$, from (6.44) with $\delta=\alpha$ we have

$$
\frac{1}{n} \mathbb{E}\left[\int_{t}^{T}\left|(-A)^{\alpha}\left(u_{\varepsilon, n}\right)_{s}\right|_{H}^{2} d s\right] \leq C n^{2 \alpha-1}\left\|u_{\varepsilon}\right\|_{\mathscr{U}_{2}}^{2} \rightarrow 0, n \rightarrow+\infty,
$$

and this concludes the proof.

The following theorem constitutes the main result of the section.

Theorem 6.25. Let Hypotheses 2.1, 6.7, 6.20 hold true, and assume that Hypothesis 6.1 holds true with $\beta>\frac{1}{2}$. For any $n \in \mathbb{N}$, let $\bar{u}^{n}$ and $v_{n}$ denote respectively the process in (6.42) and the mild solution to (6.38). Let $V, J$ be respectively the functions in (6.3), (6.2). Then, for any $(t, x) \in[0, T] \times E$,

$$
V(t, x)=\lim _{n \rightarrow+\infty} v_{n}(t, x)=\lim _{n \rightarrow+\infty} J\left(t, x, \bar{u}^{n}\right) .
$$

Moreover, $\left(\bar{u}^{n}\right)$ is bounded in $\mathscr{U}_{2}$.

Proof. Let $(t, x) \in[0, T]$ and $\varepsilon>0$. For any $n \in \mathbb{N}$, let $u_{\varepsilon}, u_{\varepsilon, n}$ be the processes introduced in Definition 6.22. By Proposition 6.24, there exists $\bar{n} \in \mathbb{N}$ such that $\left|J_{n}\left(t, x, u_{\varepsilon, n}\right)-J\left(t, x, u_{\varepsilon}\right)\right| \leq \varepsilon$, for any $n \geq \bar{n}$, which in turn gives

$$
J_{n}\left(t, x, u_{\varepsilon, n}\right) \leq V(t, x)+2 \varepsilon, \quad n \geq \bar{n} .
$$


Notice that, from the definitions of $V, V_{n}$ in (6.3), (6.37), it follows that

$$
V(t, x) \leq V_{n}(t, x) .
$$

Further, from (6.44) with $\delta=\alpha$ we get that $u_{\varepsilon, n} \in \mathscr{U}_{2}^{\alpha}$, and therefore the definition of $V_{n}$ implies

$$
V_{n}(t, x) \leq J_{n}\left(t, x, u_{\varepsilon, n}\right), \quad n \geq \bar{n} .
$$

Then, collecting (6.47), (6.48) and (6.49), $V(t, x) \leq V_{n}(t, x) \leq J_{n}\left(t, x, u_{\varepsilon, n}\right) \leq V(t, x)+2 \varepsilon$ for any $n \geq \bar{n}$. Hence, $V(t, x) \leq \lim \sup _{n \rightarrow+\infty} V_{n}(t, x) \leq V(t, x)+2 \varepsilon$, and the arbitrariness of $\varepsilon$ gives

$$
\lim _{n \rightarrow+\infty} V_{n}(t, x)=V(t, x) .
$$

Then the first equality in (6.46) follows from (6.50), recalling that, by Theorem $6.21, V_{n}(t, x)=v_{n}(t, x)$ for any $n \in \mathbb{N}$.

On the other hand, since $V_{n}(t, x)=J_{n}\left(t, x, \bar{u}^{n}\right)$ for any $n \in \mathbb{N}$,

$$
V(t, x) \leq J\left(t, x, \bar{u}^{n}\right) \leq J_{n}\left(t, x, \bar{u}^{n}\right)=V_{n}(t, x),
$$

so that, taking into account (6.50), the second equality in (6.46) follows.

Finally, let us prove that $\left(\bar{u}^{n}\right)$ is bounded in $\mathscr{U}_{2}$. Assume by contradiction that there exists a subsequence $\left(u_{k_{n}}\right) \subset\left(\bar{u}^{n}\right)$ such that $\left\|u_{k_{n}}\right\|_{\mathscr{Q}_{2}}^{2} \geq n$ for any $n \in \mathbb{N}$. Then,

$$
\begin{aligned}
n & \leq\left\|u_{k_{n}}\right\|_{\mathscr{U}_{2}}^{2}=\int_{t}^{T}\left(\int_{\left\{\left|u_{k_{n}}(s)\right|_{H} \leq R\right\}}\left|u_{k_{n}}(s)\right|^{2} d \mathbb{P}\right) d s+\int_{t}^{T}\left(\int_{\left\{\left|u_{k_{n}}(s)\right|_{H}>R\right\}}\left|u_{k_{n}}(s)\right|^{2} d \mathbb{P}\right) d s \\
& \leq T R^{2}+\int_{t}^{T}\left(\int_{\left\{\left|u_{k_{n}}\right|{ }_{H}>R\right\}}\left|u_{k_{n}}(s)\right|^{2} d \mathbb{P}\right) d s .
\end{aligned}
$$

On the other hand, since $\ell$ is nonnegative and satisfies (6.22),

$$
\begin{aligned}
& \mathbb{E}\left[\int_{t}^{T} \ell\left(s, X_{s}^{k_{n}}, u_{k_{n}}(s)\right) d s\right] \\
& =\int_{t}^{T}\left(\int_{\left\{\left|u_{k_{n}}(s)\right|_{H} \leq R\right\}} \ell\left(s, X_{s}^{k_{n}}, u_{k_{n}}(s)\right) d \mathbb{P}\right) d s+\int_{t}^{T}\left(\int_{\left\{\left|u_{k_{n}}(s)\right|_{H}>R\right\}} \ell\left(s, X_{s}^{k_{n}}, u_{k_{n}}(s)\right) d \mathbb{P}\right) d s \\
& \geq \int_{t}^{T}\left(\int_{\left\{\left|u_{k_{n}}(s)\right|_{H}>R\right\}}\left|u_{k_{n}}(s)\right|^{2} d \mathbb{P}\right) d s .
\end{aligned}
$$

Therefore $\mathbb{E}\left[\int_{t}^{T} \ell\left(s, X_{s}^{k_{n}}, u_{k_{n}}(s)\right) d s\right] \geq n-T R^{2}$, which contradicts (6.46).

\section{References}

[1] J. M. Bismut. Martingales, the Malliavin calculus and hypoellipticity under general Hörmander's conditions. Z. Wahrsch. Verw. Gebiete, 56:469-505, 1981.

[2] P. Briand and F. Confortola. BSDEs with stochastic lipschitz condition and quadratic PDE s in Hilbert spaces. Stochastic Process. Appl., 118(5):818-838, 2008.

[3] S. Cerrai. Second order PDE's in finite and infinite dimension, A probabilistic approach, volume 1762 of Lecture Notes in Mathematics. Springer-Verlag, Berlin, 2001. 
[4] G. Da Prato and J. Zabczyk. Ergodicity for infinite-dimensional systems, volume 229 of London Mathematical Society Lecture Note Series. Cambridge University Press, Cambridge, 1996.

[5] G. Da Prato and J. Zabczyk. Second order partial differential equations in Hilbert spaces., volume 293 of London Mathematical Society Note Series. Cambridge University Press, Cambridge, 2002.

[6] G. Da Prato and J. Zabczyk. Stochastic equations in infinite dimensions, volume 152 of Encyclopedia of Mathematics and its Applications. Cambridge University Press, Cambridge, second edition, 2014.

[7] F. Delbaen, Y. Hu, and X. Bao. Backward SDEs with superquadratic growth. Probab. Theory Related Fields, 150(1-2):145-192, 2011.

[8] S. El Karoui, N. Peng and M. C. Quenez. Backward stochastic differential equations in finance. Mathematical finance, 7(1):1-71, 1997.

[9] G. Fabbri, F. Gozzi, and A. Święch. Stochastic optimal control in infinite dimensions: Dynamic programming and HJB equations, with Chapter 6 by M. Fuhrman and G. Tessitore. Springer, 2017.

[10] M. Fuhrman and G. Tessitore. The Bismut-Elworthy formula for backward SDEs and applications to nonlinear Kolmogorov equations and control in infinite dimensional spaces. Stoch. Stoch. Rep., 74(1-2):429-464, 2002.

[11] M. Fuhrman and G. Tessitore. Nonlinear Kolmogorov equations in infinite dimensional spaces: the backward stochastic differential equations. Ann. Probab., 30(3):1397-1465, 2002.

[12] M. Fuhrman and G. Tessitore. Generalized Directional Gradients, Backward Stochastic Differential Equations and Mild Solutions of Semilinear Parabolic Equations. Appl Math Optim., 51(3):279-332, 2005.

[13] Y. Fuhrman, M. Hu and G. Tessitore. On a class of stochastic optimal control problems related to BSDEs with quadratic growth. SIAM Journal on Control and Optimization., 45(4):1279-1296, 2006.

[14] F. Gozzi. Regularity of solutions of second order Hamilton-Jacobi equations in Hilbert spaces and applications to a control problem. 20, 1995.

[15] F. Gozzi. Global regular solutions of second order Hamilton-Jacobi equations in Hilbert spaces with locally Lipschitz nonlinearities. 198, 1996.

[16] M. Kobylanski. Backward stochastic differential equations and partial differential equations with quadratic growth. Ann. Probab., 28(2):558-602, 2000.

[17] A. Lunardi. Analytic semigroups and optimal regularity in parabolic problems. Modern Birkhäuser Classics. Birkhäuser/Springer Basel AG, Basel, 1995. [2013 reprint of the 1995 original] [MR1329547].

[18] F. Masiero. Regularizing properties for transition semigroups and semilinear parabolic equations in Banach spaces. Electron. J. Probab., 12:no. 13, 387-419, 2007.

[19] F. Masiero. Stochastic optimal control problems and parabolic equations in Banach spaces. SIAM J. Control Optim., 47(1):251-300, 2008.

[20] F. Masiero. Hamilton Jacobi Bellman equations in infinite dimensions with quadratic and superquadratic Hamiltonian. Discrete and Continuous Dynamical Systems, 32(1A):223-263, 2012.

[21] F. Masiero. A Bismut-Elworthy formula for quadratic BSDEs. Stochastic Process. Appl., 125(5):1945-1979, 2015. 
[22] F. Masiero and A. Richou. HJB equations in infinite dimensions with locally lipschitz hamiltonian and unbounded terminal condition. J. Differential Equations, 257(6):1989-2034, 2014.

[23] É. Pardoux and S. Peng. Backward stochastic differential equations and quasilinear parabolic partial differential equations. In Stochastic partial differential equations and their applications (Charlotte, NC, 1991), volume 176 of Lect. Notes Control Inf. Sci., pages 200-217. Springer, Berlin, 1992.

[24] K. R. Parthasarathy. Probability measures on metric spaces, volume 3 of Probability and Mathematical Statistics. Academic Press, Inc., New York-London, 1967.

[25] S. Peszat and J. Zabczyk. Strong Feller property and irreducibility for diffusions on Hilbert spaces. Ann. Probab., 23(1):157-172, 1995.

[26] A. Richou. Markovian quadratic and superquadratic BSDEs with an unbounded terminal condition. Stochastic Process. Appl., 122(9):3173-3208, 2012. 